\title{
Quasinormal modes of massive charged flavor branes
}

\author{
Matthias Kaminski, ${ }^{a, b}$ Karl Landsteiner, ${ }^{a}$ Francisco Pena-Benitez, ${ }^{a}$ \\ Johanna Erdmenger, ${ }^{c}$ Constantin Greubel $^{c}$ and Patrick Kerner ${ }^{c}$ \\ ${ }^{a}$ Instituto de Física Teórica CSIC/UAM, C-XVI Universidad Autónoma de Madrid, \\ E-28049 Madrid, Spain \\ ${ }^{b}$ Department of Physics, Princeton University, \\ Princeton, NJ 08544, U.S.A. \\ ${ }^{c}$ Max-Planck-Institut für Physik (Werner-Heisenberg-Institut), \\ Föhringer Ring 6, 80805 München, Germany \\ E-mail: mkaminsk@princeton.edu, karl.landsteiner@uam.es, \\ fran.penna@uam.es, jke@mppmu.mpg.de, greubel@mppmu.mpg.de, \\ pkerner@mppmu.mpg.de
}

ABSTRACT: We present an analysis and classification of vector and scalar fluctuations in a D3/D7-brane setup at finite temperature and baryon density. The system is dual to an $\mathcal{N}=2$ supersymmetric Yang-Mills theory with $\mathrm{SU}\left(N_{c}\right)$ gauge group and $N_{f}$ hypermultiplets in the fundamental representation in the quenched approximation. We improve significantly over previous results on the quasinormal mode spectrum of D7-branes and stress their novel physical interpretation. Amongst our findings is a new purely imaginary scalar mode that becomes tachyonic at sufficiently low temperature and baryon density. We establish the existence of a critical density above which the scalar mode stays in the stable regime for all temperatures. In the vector sector we study the crossover from the hydrodynamic to the quasiparticle regime and find that it moves to shorter wavelengths for lower temperatures. At zero baryon density the quasinormal modes move toward distinct discrete attractor frequencies that depend on the momentum as we increase the temperature. At finite baryon density, however, the trajectories show a turning behavior such that for low temperature the quasinormal mode spectrum approaches the spectrum of the supersymmetric zero temperature normal modes. We interpret this as resolution of the singular quasinormal mode spectrum that appears at the limiting D7-brane embedding at vanishing baryon density.

KEYwORDS: Gauge-gravity correspondence, Black Holes in String Theory, D-branes, AdSCFT Correspondence

ArXIV EPRINT: 0911.3544 


\section{Contents}

1 Introduction and summary 1

2 Quasinormal modes and holography 5

3 Holographic setup $\quad 6$

3.1 The $\rho$-coordinate system 6

$\begin{array}{lll}3.2 & \text { Brane setup and background fields in } z \text {-coordinates } & 7\end{array}$

4 Vanishing momentum and density 10

$\begin{array}{lll}4.1 & \text { Transverse vectors } & 10\end{array}$

$\begin{array}{ll}4.2 \text { Longitudinal vectors } & 10\end{array}$

$\begin{array}{lll}4.3 \text { Scalar } & 12\end{array}$

$\begin{array}{lll}4.4 & \text { Schrödinger potential analysis } & 15\end{array}$

$\begin{array}{ll}\text { 4.5 Discussion: tachyon and de-singularization } & 15\end{array}$

5 Finite momentum but vanishing density $\quad 16$

$\begin{array}{ll}5.1 \text { Transverse vectors } & 16\end{array}$

$\begin{array}{ll}5.2 \text { Longitudinal vectors } & 18\end{array}$

$\begin{array}{lll}5.3 & \text { Scalar } & 19\end{array}$

$\begin{array}{ll}5.4 \text { Schrödinger potential analysis } & 19\end{array}$

5.5 Discussion: breakdown of hydrodynamics, 'attractors' and the tachyon 21

5.5.1 Hydrodynamics to collisionless crossover 22

5.5.2 "Attractor" frequencies 24

$\begin{array}{ll}\text { 5.5.3 Tachyon: a new hydrodynamic mode } & 25\end{array}$

6 Finite density but vanishing momentum 25

$\begin{array}{lll}6.1 & \text { Transverse vectors } & 26\end{array}$

$\begin{array}{lll}6.2 & \text { Scalar } & 27\end{array}$

$\begin{array}{lll}6.3 & \text { Schrödinger potential analysis } & 29\end{array}$

6.4 Analytic solution at high frequencies 34

6.5 Discussion: turning point and tachyon 38

$\begin{array}{lll}\text { 6.5.1 Turning point } & 38\end{array}$

6.5.2 Killing the tachyon 42

7 Conclusions and outlook $\quad 42$

$\begin{array}{ll}\text { A Shooting method } & 43\end{array}$

$\begin{array}{ll}\text { B Relaxation method } & 44\end{array}$

$\begin{array}{ll}\text { C Schrödinger potentials } & 47\end{array}$ 
E Results for second quasinormal modes $\quad \mathbf{5 0}$

E.1 Transverse vector fluctuations $\quad 50$

$\begin{array}{ll}\text { E.2 Longitudinal vector fluctuations } & 51\end{array}$

$\begin{array}{lll}\text { E.3 Scalar fluctuations } & 52\end{array}$

\section{Introduction and summary}

One of the successes of the AdS/CFT correspondence [1,2] and its generalizations is its application to the plasma phase of non-Abelian gauge theories [3]. This is of high interest because of its potential relevance for the description of the strongly-coupled quark-gluon plasma as it is created in Heavy Ion Collision at RHIC or in the near future at the LHC.

The holographic modelling of the plasma phase invariably involves an asymptotically AdS black hole [4]. Of particular interest are the quasinormal modes of such black holes as they are mapped to the poles in the correlation function of the dual finite temperature field theory $[5,6]$. One aspect of this is a relation between the quasinormal frequencies and the hydrodynamic transport coefficients, e.g. the shear viscosity.

An important generalization of the AdS/CFT correspondence is the addition of flavor degrees of freedom in the fundamental representation of the gauge group. One convenient way of achieving this is via the addition of D7-brane probes to the ten-dimensional supergravity background [7]. The meson spectrum can then be studied via the fluctuations of the probe brane $[8,9]$.

For the configuration of a D7-brane probe added to the AdS Schwarzschild black hole background, a first order phase transition occurs between D7-brane probes either staying outside of the horizon or reaching down to it [10-12]. These two types of embeddings are called Minkowski or black hole embeddings, respectively. As discussed in [13], the first case corresponds to stable mesons, whereas in the second case the mesons are unstable. For stable mesons, the spectrum has been determined analytically at zero temperature in [8]. In the second case, the meson excitations may be identified with quasinormal modes and their finite width is related to an infalling energy boundary condition at the black hole horizon. The spectral functions for this configuration were first studied in [14]. If the temperature is high compared to the quark mass the quasinormal frequencies lie deep inside the lower complex half plane and the spectral function is smooth and without noticeable peaks. As the temperature is lowered the quasinormal modes move towards the real axis, producing rather well defined quasiparticle peaks in the spectral function. This behavior is particularly strong at finite baryon density.

Furthermore a tachyonic mode is present in the scalar sector close to the first order phase transition discussed above. For null momentum, the spectral function of this configuration has been studied in [15] and the presence of the tachyonic mode has been argued for by way of a WKB analysis. We find indeed a so far overlooked purely imaginary 
mode in the scalar spectrum that crosses the real axis and therefore becomes unstable at a temperature that is in excellent numerical agreement with [15].

At finite baryon chemical potential, as obtained by considering a non-zero profile for the time component of the gauge field on the D7-brane, there is a rich phase structure for the D7-brane embedded in the AdS Schwarzschild background [16-20]. In these works the strict supergravity limit is considered, i.e. $\alpha^{\prime} \rightarrow 0$, and the results are thus perturbative on the gravity side. Non-perturbative effects such as worldsheet instantons have been studied in [21]. Taking these effects into account the first-order phase transition mentioned above becomes third order at a critical chemical potential [21]. In this paper however we will consider the strict supergravity limit only.

The spectral functions for vector mesons at finite baryon density have been studied in [22], where a particular turning behavior of the quasi-normal frequencies in the complex frequency plane was observed as function of the ratio of quark mass over temperature: at low values of $M_{q} / T$, the position of the quasinormal modes moves to smaller real parts when $M_{q} / T$ increases, while for large values, it moves to larger real parts. For large $M_{q} / T$, the spectrum approaches the form of the supersymmetric case. The momentum dependence of the spectral function at finite baryon density has been investigated in [23, 24].

Summary of results. The purpose of the present paper is to present an in depth study of both scalar and vector modes at either finite baryon density or finite momentum.

At zero density and zero momentum we determine the first and second quasinormal frequency of the scalar and vector fluctuations. We calculate their trajectories in the complex plane as we increase the quark mass over temperature ratio. In general these modes move along a curve where initially the real and imaginary parts can grow a little but then move continuously towards lower values. As shown in [25] the endpoints of these curves asymptote to a single point on the real frequency axis that is reached as the embedding reaches the limiting embedding. This happens when the brane just touches the black hole horizon. ${ }^{1}$ There it has been argued that the spectrum of quasinormal modes becomes singular as they all coincide at one (real) energy value. It is however unclear if this limiting embedding can really be reached since the embeddings become locally unstable at rather high temperatures. Indeed in the scalar sector we find a mode that becomes tachyonic shortly after the system has become thermodynamically metastable due to the presence of the first order phase transition. Once the tachyonic mode is in the spectrum the embedding is not even metastable and so far it is not known what the true ground state would be or if such a state exists at all.

At finite momentum but zero density, we find that at a critical momentum the system undergoes a crossover transition from the hydrodynamic behavior at long wavelengths to a collisionless behavior at small wavelengths. We observe this crossover in the longitudinal vector channel which has a hydrodynamic mode, i.e. a mode whose dispersion relation does not show a gap at zero momentum. This mode describes baryon charge diffusion and

\footnotetext{
${ }^{1}$ Note that this limiting embedding is often called critical embedding in the literature e.g. [12]. However in this paper we use the term 'critical embedding' for the embedding at which the phase transition occurs (cf. figure 1 a).
} 
is thus called diffusion mode. The transition from the hydrodynamic to the collisionless regime is defined as the point where the imaginary part of the diffusion mode becomes larger than the imaginary part of the first quasinormal mode which is not a hydrodynamic mode. Additionally we observe that the first quasinormal mode of the scalar and vector fluctuations with increasing momentum show an increasing number of spirals in their trajectories parametrized by the quark mass over temperature ratio. The number $n$ of these spirals apparently is correlated with certain "attractor" frequencies, i.e. the real part of the quasinormal modes asymptotes to $\omega_{n} \in \mathbf{R}$ as the D7-brane embedding approaches the critical embedding between the black hole and Minkowski embeddings. Although the asymptotic values $\omega_{n}$ lie deep in the unstable sector, the spirals mostly lie in the physical sector of the theory. So they are physical features of the (meta)stable theory.

At finite quark density but zero momentum, we find the following: in the vector mode sector, there are two distinct movements of the quasinormal frequencies in dependence of the quark mass over temperature ratio. At small densities there is a turning point as already observed in [22] and as described above. This turning point is no longer present for large densities. The critical value for the normalized density is $\tilde{d}_{c}=0.04$. The relation between the normalized density $\tilde{d}$ and the baryon density $n_{B}$ is given by

$$
\tilde{d}=\frac{2^{\frac{5}{2}} n_{B}}{N_{f} \sqrt{\lambda} T^{3}}
$$

where $T$ is the temperature, $N_{f}$ the number of flavors and $\lambda$ the 't Hooft coupling. In the scalar channel, the quasinormal frequencies show also distinct movements in dependence of the quark mass over temperature ratio. In this case we find three distinct behaviors separated by critical densities. As a function of $M_{q} / T$, the quasinormal modes perform either a left turn, a right turn or no turn in the complex frequency plane. We will study these different behaviors in detail.

For the scalar channel, we find in addition that the tachyonic mode mentioned above is only present below a critical normalized density, $\tilde{d}<0.00315$. We identify the unstable region in which the tachyon is present with an instability related to the first order phase transition found in the thermodynamics of the system. This behavior may be understood by comparing it to the first order phase transition of the well-known van-der-Waals gas, as described for instance in [26]. Figure 1 (b) shows the first order phase transition of the vander-Waals gas, which follows the red line in the $p$ - $V$-diagram as obtained from the Maxwell construction. Additionally there are two metastable branches: one of them reaches from the point where the phase transition begins when increasing the volume to the minimum denoted by the red plus sign in the figure. The second reaches from the maximum denoted by the red cross to the point where the phase transition ends when increasing the volume. The region between the two extrema is unstable since the pressure raises when the volume is increased.

Our calculations show a very similar behavior of the system considered here. We have calculated both the free energy as function of $m \propto M_{q} / T$ close to the phase transition, ${ }^{2}$

\footnotetext{
${ }^{2} \mathrm{~A}$ similar calculation of the free energy was performed in [16].
} 


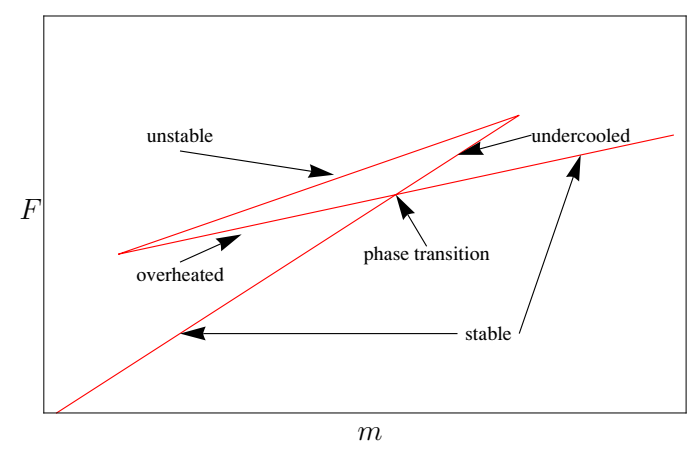

(a)

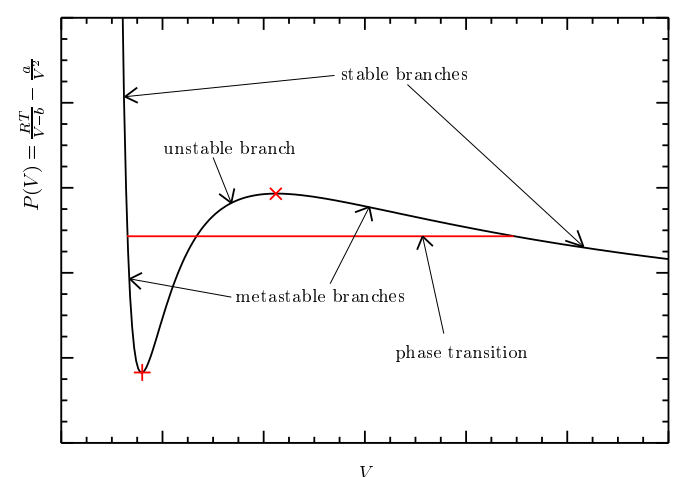

(b)

Figure 1. (a) Sketch of the free energy $F$ of the flavor fields versus the quark mass over temperature ratio $m \propto M_{q} / T$ close to the first order phase transition. (b) Pressure versus volume of the van-derWaals gas. The red line marks the phase transition which is obtained by the Maxwell construction.

displayed schematically in figure 1 (a), and the quasinormal modes for the same range of $M_{q} / T$. Both calculations show a similar structure of stable, metastable and unstable branches, with the numerical values for the boundaries of these branches agreeing up to four digits in both calculations. In particular in analogy to the van-der-Waals gas we find that in addition to the stable branches ${ }^{3}$ there are metastable branches close to the phase transition, denoted as overheated and undercooled ${ }^{4}$ in figure 1 (a). Furthermore we find an unstable branch which connects these two metastable branches. The metastable phases are stable against fluctuations, while on the unstable branch a tachyonic mode appears in the quasinormal spectrum. In particular we emphasize that at zero density the limiting embedding which has a conical singularity at the black hole horizon, and is often discussed in the literature, lies clearly deep inside the unstable region. Thus any observation found by using this embedding should be considered with great care since it does not correspond to a physical state. Furthermore we should stress that on the field theory side there exists a stable ground state for any combination of the mass $m$ and chemical potential $\mu$.

We also present a qualitative analysis of the quasinormal spectrum at either finite momentum or finite density which uses the fact that the equation of motion for the fluctuations can be transformed into a Schrödinger equation. The Schrödinger potential analysis was introduced for instance in [13, 14, 23, 25].

The results of this analysis are in qualitative agreement with the results obtained by stating the quasinormal modes listed above: in particular, at finite density we find a general feature in the Schrödinger potential: at low quark mass over temperature ratio there are only unbound scattering states which correspond to quasinormal modes. By increasing the quark mass over temperature ratio, a barrier forms in the Schrödinger potential [23]

\footnotetext{
${ }^{3}$ Note that in [16] it was observed that for a small region of the stable phase in which the mass parameter $m$ is just slightly larger than the critical one there is an charge instability. This instability is distinct from the instability discussed above. We do not observe this charge instability in our quasinormal mode analysis. This may be due to inadequacy of the our numerical methods at large mass over temperature ratios.

${ }^{4}$ In the context of the D3/D7 system, the terms 'overheated' and 'undercooled' were introduced in [25].
} 
which leads to a local minimum. In [25] it was found for Minkowski embeddings that the Schrödinger potential is a box whose extent in the AdS radial direction coincides with the region filled by the D7-brane probe. Here we observe that for black hole embeddings at finite density, a barrier forms in the Schrödinger potential at the same radial position as the IR boundary of the box in the Minkowski case. This barrier separates the bulk of the AdS space from the horizon of the black hole. As the barrier increases when the ratio of quark mass over temperature $m \propto M_{q} / T$ raises, less energy can leak into the black hole. Thus 'bound' states which correspond to 'normal' modes are formed. We study the appearance and the behavior of this barrier in detail.

The paper is organized as follows: in section 2 we give a general introduction to the relation between quasinormal modes and hydrodynamics. In section 3 we introduce the D3/D7-brane setup. In the sections 4,5 and 6 we determine the quasinormal modes of the scalar and vector fluctuations first at vanishing momentum and density and later at finite momentum or finite density. In every section we also calculate the corresponding Schrödinger potentials which we use to describe the qualitative behavior of the quasinormal modes. At the end of each section we summarize the physical results which we found.

\section{Quasinormal modes and holography}

In this section we recall the definition of quasinormal modes of black holes and the role they play in determining the response of a holographic field theory close to equilibrium.

Quasinormal modes of a black hole are distinct perturbations of the black hole solution. Roughly they can be understood as resonances of the black hole. However since the energy of the perturbation can leak into the black hole, these fluctuations are not normal modes and thus have been dubbed quasinormal. Their corresponding frequencies consist of a real and an imaginary part. As for the damped oscillator, the real part of the frequency essentially determines the energy of the fluctuations, while the imaginary part is responsible for the damping. In AdS spacetimes the quasinormal modes satisfy the following boundary conditions. At the horizon they are purely ingoing, whereas at the conformal AdS boundary they have an asymptotic behavior that corresponds to a normalizable mode. In this paper we determine the quasinormal spectrum of the D7-branes. The corresponding modes can be grouped in terms of their transformation properties under spatial rotations of $\mathrm{SO}(3)$. We consider scalar modes given by perturbations of the brane embedding as well as vector modes given by perturbations of the gauge field on the brane. As is well established by now, the quasinormal frequencies of the dual gravity theory can be identified with the poles of correlation functions in dual thermal gauge theories $[5,6]$.

A system which is close to equilibrium can be described by linear response theory. There the effect of an external perturbation is given by the retarded two point function folded against the source of the perturbation. By a Cauchy integration in the complex frequency plane the response can be written as a sum over the contributions of the different quasinormal modes (see e.g. [27]). Writing the time dependence as $\exp (-i \omega t)$ we note that a relaxation towards equilibrium can only happen if all the quasinormal modes lie in the lower complex half plane. Following $[27,28]$ the response caused by an external perturbation 
$\tilde{\jmath}(\omega, k)$ can therefore be written as a sum over quasinormal frequencies

$$
\langle\Phi(t, k)\rangle=i \theta(t) \sum_{n} R_{n} \tilde{\jmath}\left(\omega_{n}, k\right) e^{-i \Omega_{n} t-\Gamma_{n} t},
$$

where the quasinormal frequencies are $\omega_{n}=\Omega_{n}-i \Gamma_{n}$ and their residues are $R_{n}$. If a mode comes to lie in the upper half plane it results in an exponentially growing mode and therefore represents an instability of the system. As we will see in the following, such an instability does indeed occur in the scalar sector of the D7-brane fluctuations.

Of particular interest is the hydrodynamic limit that considers only fluctuations with small frequency and large wavelength. This hydrodynamic expansion can be seen as an effective field theory where the degrees of freedom with large frequency and small wavelength are integrated out. In this effective field theory only the poles of correlation functions closest to the origin are important. More precisely, the modes with a dispersion relation obeying $\lim _{\vec{k} \rightarrow 0} \omega(\vec{k})=0$ represent the hydrodynamic regime. Hydrodynamic transport coefficients such as the shear viscosity or the diffusion constants can be read off form these poles. Finally let us mention that recently it has been shown that the determinant of wave operators in some asymptotically black hole backgrounds can be written in terms of the quasinormal modes [29].

\section{$3 \quad$ Holographic setup}

We are interested in a large $N_{c}$ thermal field theory, at finite baryon chemical potential including fundamental and adjoint matter. Our matter content is that of the $\mathcal{N}=4$ super Yang-Mills theory and a number $N_{f}$ of $\mathcal{N}=2$ super Yang-Mills fundamental hypermultiplets. We consider the quenched approximation with $N_{c} \rightarrow \infty$ and $N_{f} \ll N_{c}$ fixed. In this limit the theory stays conformal at leading order in $N_{c}$.

The dual gravity setup is given by a stack of $N_{c}$ D3-branes and $N_{f}$ probe D7-branes. The metric generated by the D3-branes is a non-extremal AdS black hole background placing the field theory at finite temperature. The D7-branes allow for strings which have one end on a D3- and the other end on the D7-brane. These 3-7 strings correspond to quarks in the fundamental representation. The length of these strings, i.e. the separation between the stacks of D3- and D7-branes corresponds to the quark mass $M_{q}$ in the field theory. On the world volume of the D7-branes we introduce a background gauge field $A$ which generates a chemical potential $\mu$ in the field theory at the boundary. Depending on the problem it is convenient to work in different coordinate systems. We now introduce the $\rho$-coordinates and the $z$-coordinates.

\subsection{The $\rho$-coordinate system}

We find it convenient to use the $\rho$-coordinates of [16] to write the AdS black hole background in Minkowski signature as

$$
d s^{2}=\frac{\varrho^{2}}{2 R^{2}}\left(-\frac{f^{2}}{\tilde{f}} \mathrm{~d} t^{2}+\tilde{f} \mathrm{~d} \vec{x}^{2}\right)+\left(\frac{R}{\varrho}\right)^{2}\left(\mathrm{~d} \varrho^{2}+\varrho^{2} \mathrm{~d} \Omega_{5}^{2}\right),
$$


with $\mathrm{d} \Omega_{5}^{2}$ the metric of the unit 5 -sphere and

$$
f(\varrho)=1-\frac{\varrho_{H}^{4}}{\varrho^{4}}, \quad \tilde{f}(\varrho)=1+\frac{\varrho_{H}^{4}}{\varrho^{4}},
$$

where $R$ is the AdS radius, with

$$
R^{4}=4 \pi g_{s} N_{c} \alpha^{2}=2 \lambda \alpha^{2}
$$

This type of radial coordinate is better suited for calculation of the quasinormal modes with the shooting method, where one places a cutoff at some large value of $\rho$.

The temperature of the black hole given by (3.1) may be determined by demanding regularity of the Euclidean section. It is given by

$$
T=\frac{\varrho_{H}}{\pi R^{2}} .
$$

In the following we may use the dimensionless coordinate $\rho=\varrho / \varrho_{H}$, which covers the range from the event horizon at $\rho=1$ to the boundary of the AdS space at $\rho \rightarrow \infty$.

To write down the DBI action for the D7-branes, we introduce spherical coordinates $\left\{r, \Omega_{3}\right\}$ in the 4567-directions and polar coordinates $\{L, \phi\}$ in the 89-directions [16]. The angle between these two spaces is denoted by $\Theta(0 \leq \Theta \leq \pi / 2)$. The six-dimensional space in the 456789-directions is given by

$$
\begin{aligned}
\mathrm{d} \varrho^{2}+\varrho^{2} \mathrm{~d} \Omega_{5}^{2} & =\mathrm{d} r^{2}+r^{2} \mathrm{~d} \Omega_{3}^{2}+\mathrm{d} L^{2}+L^{2} \mathrm{~d} \phi^{2} \\
& =\mathrm{d} \varrho^{2}+\varrho^{2}\left(\mathrm{~d} \Theta^{2}+\cos ^{2} \Theta \mathrm{d} \phi^{2}+\sin ^{2} \Theta \mathrm{d} \Omega_{3}^{2}\right),
\end{aligned}
$$

where $r=\varrho \sin \Theta, \varrho^{2}=r^{2}+L^{2}$ and $L=\varrho \cos \Theta$. Due to the symmetry, the embedding of the D7-branes only depends on the radial coordinate $\rho$. Defining $\chi=\cos \Theta$, we parametrize the embedding by $\chi=\chi(\rho)$ and choose $\phi=0$ using the $O(2)$ symmetry in the 89-direction. The induced metric $G$ on the D7-brane probes is then

$$
d s^{2}(G)=\frac{\varrho^{2}}{2 R^{2}}\left(-\frac{f^{2}}{\tilde{f}} \mathrm{~d} t^{2}+\tilde{f} \mathrm{~d} \vec{x}^{2}\right)+\frac{R^{2}}{\varrho^{2}} \frac{1-\chi^{2}+\varrho^{2}\left(\partial_{\varrho} \chi\right)^{2}}{1-\chi^{2}} \mathrm{~d} \varrho^{2}+R^{2}\left(1-\chi^{2}\right) \mathrm{d} \Omega_{3}^{2} .
$$

The square root of the determinant of $G$ is given by

$$
\sqrt{-G}=\frac{\sqrt{h_{3}}}{4} \varrho^{3} f \tilde{f}\left(1-\chi^{2}\right) \sqrt{1-\chi^{2}+\varrho^{2}\left(\partial_{\varrho} \chi\right)^{2}},
$$

where $h_{3}$ is the determinant of the 3 -sphere metric.

\subsection{Brane setup and background fields in $z$-coordinates}

Here we introduce the coordinates also used in [13]. This set of coordinates maps the compact interval $[0,1]$ to the distance between the conformal boundary and the black hole horizon. Such a compact radial coordinate is particularly well suited for the calculation of quasinormal modes using the relaxation method as explained in appendix B. We work 
in a non-extremal black-hole background generated by the stack of D3-branes giving the effective metric

$$
\frac{\mathrm{d} s^{2}}{R^{2}}=\frac{1}{z^{2}}\left[-f(z) \mathrm{d} t^{2}+\frac{\mathrm{d} z^{2}}{f(z)}+\mathrm{d} \vec{x}^{2}\right]+\mathrm{d} \Omega_{5}^{2},
$$

with $f(z)=1-z^{4}$. Note that the horizon is located at $z=1$, the AdS-boundary at $z=0$. Frequencies and momenta measured in (3.8) are related to physical frequencies and momenta by $\left(\omega_{p h}, k_{p h}\right)=\pi T(\omega, k)$, where $T$ is the temperature.

The D7-brane action reads

$$
S=\int \mathrm{d}^{8} \xi \sqrt{-\operatorname{det}\left(P[G]+2 \pi \alpha^{\prime} F\right)},
$$

where $P[G]$ is the metric's pull-back on the $N_{f}$ D7-branes. Here the induced metric is given by

$$
\frac{\mathrm{d} s_{D 7}^{2}}{R^{2}}=-\frac{f(z)}{z^{2}} \mathrm{~d} t^{2}+\left(\frac{1}{z^{2} f(z)}-\Theta^{\prime}(z)\right) \mathrm{d} z^{2}+\frac{1}{z^{2}} \mathrm{~d} \vec{x}^{2}+\sin ^{2} \Theta(z) \mathrm{d} \Omega_{3}^{2},
$$

where $\Theta(z)$ describes the D7-brane embedding in the AdS-Schwarzschild background and the embedding coordinates are $x^{a}=\left(t, \vec{x}, z, \alpha_{1}, \alpha_{2}, \alpha_{3}\right)$. To second order in the field strength the D7-brane Lagrangian can be written in the following way

$$
\mathcal{L}=\sqrt{-P[G]}\left[1+\pi^{2} \alpha^{\prime 2} F_{a b} F^{a b}\right] .
$$

With the zero order term, we obtain the background equation of motion

$$
\begin{aligned}
0= & 3 \cos \Theta(z)\left[-1+z^{2}\left(-1+z^{4}\right) \Theta^{\prime}(z)^{2}\right] \\
& -z \sin \Theta(z)\left[\left(3+z^{4}\right) \Theta^{\prime}(z)+2 z^{2}\left(1-z^{4}\right)\left(2-z^{4}\right) \Theta^{\prime}(z)^{3}-z\left(-1+z^{4}\right) \Theta^{\prime \prime}(z)\right] .
\end{aligned}
$$

The brane embedding can be found by integrating this equation from the horizon out to the boundary. As initial conditions one chooses $\chi_{0}=\cos (\Theta(1))$ and demands regularity on the horizon. The quark mass can be read off from the behaviour at radial AdS infinity located at $z=0[13]$

$$
M_{q}=\frac{1}{2} m \sqrt{\lambda} T, \quad m=\chi^{\prime}(z=0) .
$$

We have plotted the mass parameter $m$ as a function of $\chi_{0}=\cos \Theta\left(\varrho_{H}\right)$ in figure 2 . The mass is not a single valued function of $\chi_{0}$. Although $m$ is the physical parameter of the brane embedding we prefer to quote $\chi_{0}$ values instead of $m$ since much of our investigations will take place in the regime where $m$ ceases to be single valued. The change in the sign of $\partial m / \partial \chi_{0}$ is also suggestive of an instability. Indeed the maximum of $m=1.31$ is reached at $\chi_{0}=0.962$, which is precisely the value from where an unstable mode appears in the scalar sector of the quasinormal mode spectrum.

Looking at figure 3 it is obvious that at finite charge density the unstable region disappears at a critical density near $\tilde{d}_{c}=0.00315$ and for larger densities the quark mass is a monotonously increasing function of the embedding parameter $\chi_{0}$, i.e. $\chi_{0} \sim M_{q} / T$ for $\tilde{d}_{c} \geq 0.00315$. This critical value will be confirmed in our analysis of the quasinormal mode spectrum below. 


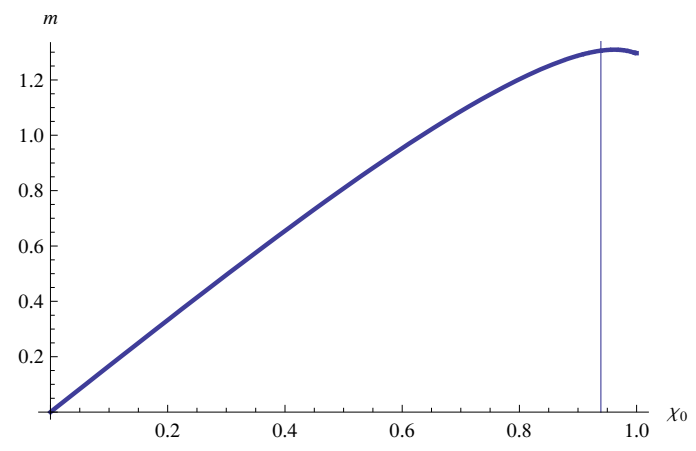

Figure 2. Plot of the dimensionless mass parameter $m$ vs the cosine $\chi_{0}=\cos \Theta_{0}$ of the embedding angle $\Theta(z)$ at the horizon, i.e. $\Theta_{0}=\Theta(z=1)$. The mass is not a single valued function of $\chi_{0}$ on $[0,1]$ ! It takes a maximum value of $m=1.31$ at $\chi_{0}=0.962$. The horizontal line indicates the first order phase transition at $\chi_{0}=0.939$.

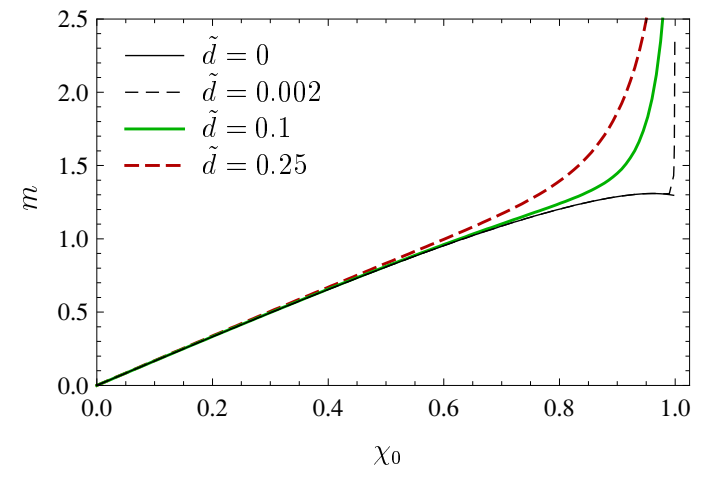

Figure 3. At finite charge density: the dependence of the dimensionless quark mass $m=$ $2 M_{q} / \sqrt{\lambda} T$ on the horizon value $\chi_{0}=\lim _{\rho \rightarrow 1} \chi$ of the embedding as shown in [30].

The second order term produces the Maxwell equation

$$
\partial_{a}\left(\sqrt{-P[G]} F^{a b}\right)=0,
$$

where $F_{a b}=\partial_{a} A_{b}-\partial_{b} A_{a}$. We can choose the $A_{z}=0$ gauge and expand $A_{a}$ in plane wave modes. Moreover if we write the equations in a gauge invariant way using the electric fields in longitudinal $E_{L}=k_{p h} A_{0}+\omega_{p h} A_{1}$ and transverse direction $\vec{E}_{T}=\omega_{p h} \vec{A}_{T}$ the equations of motion are

$$
\begin{aligned}
E_{L}^{\prime \prime}(z)+\left[C(z)+\frac{f^{\prime}(z) \omega^{2}}{f(z)\left(\omega^{2}-f(z) k^{2}\right)}\right] & E_{L}^{\prime}(z)+B(z)\left(\omega^{2}-f(z) k^{2}\right) E_{L}(z)=0 \\
E_{T}^{\prime \prime}(z)+\left[C(z)+\frac{f^{\prime}(z)}{f(z)}\right] & E_{T}^{\prime}(z)+B(z)\left(\omega^{2}-f(z) k^{2}\right) E_{T}(z)=0
\end{aligned}
$$

with

$$
\begin{aligned}
& B(z)=\frac{1}{f^{2}(z)}+\frac{z^{2} \Theta^{\prime}(z)^{2}}{f(z)}, \\
& C(z)=-\frac{1}{z}+2 z\left(-2+z^{4}\right) \Theta^{\prime}(z)^{2} .
\end{aligned}
$$

Our results are summarized in the following three sections. 


\section{Vanishing momentum and density}

Our analysis produced a considerable amount of data and we will not show all of it because of some redundancy in the results. Technical details on our numerical methods are also deferred to the appendices A and B. The idea for this and the following two sections is, to have example figures for each case of interest and a listing of all the effects we observe. We also provide a qualitative analysis by studying the correspondent Schrödinger equations. The discussion subsection in each of the cases is then devoted to the physically most interesting effects, i.e. the tachyon, diffusion mode, turning point.

\subsection{Transverse vectors}

The transverse equation of motion can be written in this simplified form

$$
E_{T}^{\prime \prime}(z)+A_{1}(z) E_{T}^{\prime}(z)+B(z)\left(\omega^{2}-f(z) k^{2}\right) E_{T}(z)=0,
$$

where $A_{1}(z)=C(z)+\frac{f^{\prime}(z)}{f(z)}$. Close to the boundary of AdS $\left(z \rightarrow 0^{+}\right)$, the differential equation reduces to

$$
E_{T}^{\prime \prime}(z)-\frac{1}{z} E_{T}^{\prime}(z)=0,
$$

which has the solution $E_{T}(z)=\mathcal{A}+\mathcal{B} z^{2}$. According the dictionary of the correspondence, $\mathcal{A}$ should be zero in order to study the quasinormal states. Close to the horizon $\left(z \rightarrow 1^{-}\right)$, the differential equation is given by

$$
E_{T}^{\prime \prime}(z)+\frac{1}{z-1} E_{T}^{\prime}(z)+\frac{\omega^{2}}{16(z-1)^{2}} E_{T}(z)=0
$$

with the solution $E_{T}(z)=\mathcal{A}^{\prime}(1-z)^{i \omega / 4}+\mathcal{B}^{\prime}(1-z)^{-i \omega / 4}$. The infalling boundary condition is fulfilled by choosing $\mathcal{A}^{\prime}=0$.

At this point we can perform the next transformation $E_{T}(z)=(1-z)^{-i \omega / 4} y(z)$, in order to split the infalling singular part from the regular part of the function. In consequence the function $y(z)$ must satisfy the boundary conditions $y(0)=0$ and $y(1)=1$ and the differential equation turns out to be

$$
y_{t}^{\prime \prime}(z)+\left[\alpha_{1}+i \omega \gamma_{1}\right] y_{t}^{\prime}(z)+\left[\alpha_{0}+i \omega \beta_{1}+\omega^{2} \beta_{2}\right] y_{t}(z)=0,
$$

with $\alpha_{1}=A_{1}, \gamma_{1}=\frac{1}{2(1-z)}, \alpha_{0}=-k^{2} f(z) B(z), \beta_{1}=\frac{1+A_{1}(1-z)}{4(1-z)^{2}}$ and $\beta_{2}=-\frac{1}{16(1-z)^{2}}+B(z)$.

Results for the quasinormal modes of the transverse vectors in this case are shown in figure 4.

\subsection{Longitudinal vectors}

The equation of motion for longitudinal vectors is given by

$$
E_{L}^{\prime \prime}(z)+\left[\frac{A_{1}(z)\left(\omega^{2}-f(z) k^{2}\right)+C_{0}(z)}{\omega^{2}-f(z) k^{2}}\right] E_{L}^{\prime}(z)+B(z)\left(\omega^{2}-f(z) k^{2}\right) E_{L}(z)=0,
$$



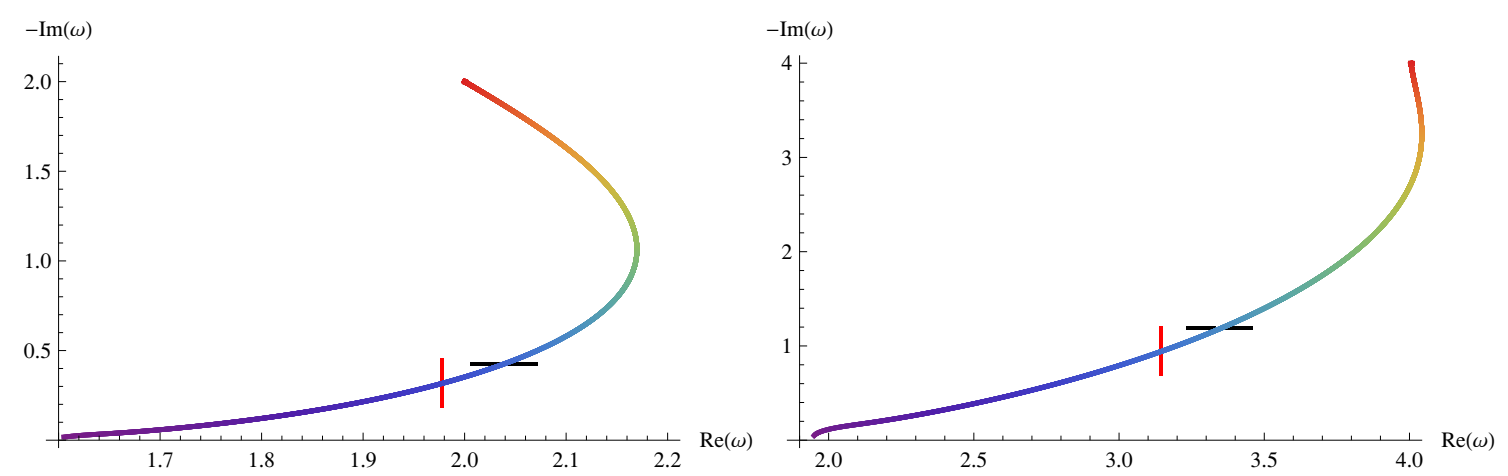

Figure 4. Location of the first (left) and second (right) quasinormal modes in the complex frequency plane for the vector fluctuations at vanishing momentum $(k=0)$ as a function of the embedding $\chi_{0}$. Red color indicates small quark mass, or high temperature, while the temperature decreases towards blue colors. The horizontal (black) dash indicates the frequency at the first order phase transition where the angle is $\chi_{0}=0.939$. The vertical (red) dash indicates the frequecy at which the embeddings become locally unstable at $\chi_{0}=0.962$. The modes are followed down to embeddings with $\chi_{0}=0.999875$.

with $C_{0}(z)=k^{2} f(z)$. The asymptotic behavior of this equation is the same for the transverse e.o.m, then if we do the same transformation that above, we obtain this equation:

$$
y_{l}^{\prime \prime}(z)+\left[\frac{\alpha_{1}^{\prime}+\alpha_{2}^{\prime} \omega^{2}}{\omega^{2}-k^{2} f(z)}+i \omega \gamma_{1}^{\prime}\right] y_{l}^{\prime}(z)+\frac{\alpha_{0}^{\prime}+\beta_{2}^{\prime} \omega^{2}+\beta_{3}^{\prime} \omega^{4}+i\left(\omega \beta_{1}^{\prime}+\omega^{3} \beta_{4}^{\prime}\right)}{\omega^{2}-k^{2} f(z)} y_{l}(z)=0
$$

with

$$
\begin{array}{ll}
\alpha_{1}^{\prime}=C_{0}(z)-A_{1}(z) k^{2} f(z), & \alpha_{2}^{\prime}=A_{1}(z), \\
\gamma_{1}^{\prime}=\frac{1}{2(1-z)}, & \alpha_{0}^{\prime}=k^{4} f^{2}(z) B(z), \\
\beta_{1}^{\prime}=\frac{C_{0}(1-z)+k^{2}\left(A_{1}(z-1)-1\right) f(z)}{4(z-1)^{2}}, & \beta_{3}^{\prime}=B(z)-\frac{1}{16(1-z)^{2}}, \\
\beta_{2}^{\prime}=\frac{k^{2}\left(1-32(-1+z)^{2} B(z)\right) f(z)}{16(-1+z)^{2}}, & \beta_{4}^{\prime}=\frac{1+A 1(z)(1-z)}{4(-1+z)^{2}} .
\end{array}
$$

In the case with $k=0$ the differential equations for transverse and longitudinal fluctuations are the same, in consequence their quasinormal spectra coincide, see figure 4 .

Results for vectors. Figure 4 shows the first and second QNM in the complex frequency plane. Starting with zero quark mass, i.e. at high temperature (red color), the imaginary part monotonously decreases with decreasing temperature. This means the corresponding mode becomes more and more stable. In contrast to that the real part of the quasinormal frequency first grows until it reaches a maximum and then decreases as well with decreasing temperature. This maximum in the real part of the QNM lies above the meson melting transition (indicated by a short horizontal dash). The melting transition takes place at a critical angle $\chi_{0}=0.939$ from which on the Minkowski embeddings are thermodynamically 


\begin{tabular}{|c|c|c|c|c|}
\hline & \multicolumn{2}{|c|}{ relaxation } & \multicolumn{2}{c|}{ shooting } \\
\hline$\chi_{0}$ & $\operatorname{Re} \omega$ & $\operatorname{Im} \omega$ & $\operatorname{Re} \omega$ & $\operatorname{Im} \omega$ \\
\hline 1st QNM & & & & \\
\hline 0 & 2.0000 & -2.0000 & 2.0000 & -2.0000 \\
\hline 0.48 & 2.1075 & -1.5973 & 2.1075 & -1.5972 \\
\hline 0.92 & 2.0656 & -0.4853 & 2.0657 & -0.4852 \\
\hline 2nd QNM & & & & \\
\hline 0 & 4.0054 & -3.9976 & 3.9995 & -4.0004 \\
\hline 0.48 & 4.0417 & -3.3366 & 3.9324 & -3.3386 \\
\hline 0.92 & 3.4397 & -1.3093 & 3.4397 & -1.3093 \\
\hline
\end{tabular}

Table 1. Exemplary values for the first and second vector QNM frequencies at $k=0$ for different values of $\chi_{0}$ parametrizing the D7-embedding. See figure 2 for the relation between $\chi_{0}$ and the quark mass $M_{q}$. The first pair of values in each row is obtained from the relaxation method, the second pair stems from requiring the shooting solution to vanish at the AdS boundary. We find a remarkable agreement.

favored, not the black hole embeddings. We have chosen to remain in the so-called undercooled phase keeping the black hole embeddings even beyond the transition. This phase is accessible since the meson-melting is a first order transition. So the undercooled phase is metastable. However we will see in the following section that at a smaller temperature below the melting transition, i.e. a larger angle $\chi_{0}^{\text {tachyon }} \approx 0.962$, this undercooled phase is destabilized by the scalar fluctuation becoming tachyonic. In the figures this is indecated by a red vertical dash.

Exemplary numerical values for the vector QNM frequencies at $k=0$ are given in table 1. We find a remarkable agreement between the values obtained with two different methods: the relaxation method and the shooting method. The results are in good agreement for all parameter regions and in all the cases we treat in this work. Therefore we exclusively show results produced with the relaxation method from now on.

\subsection{Scalar}

In the DBI-action (3.9) we let the $\Theta$-angle fluctuate and we split this fluctuation $\delta \Theta$ into a product of its singular and regular parts $\delta \Theta(z)=(1-z)^{-i \omega / 4} z y(z)$. With this change the infalling boundary condition at the horizon is translated into $y(1)=1,{ }^{5}$ and the Dirichlet condition at the boundary implies $y(0)=0$. The equation of motion for scalar fluctuations then reads

$$
y^{\prime \prime}(z)+\left[a_{1}(z)+i c_{1}(z) \omega\right] y^{\prime}(z)+\left[a_{0}(z)+i b_{1}(z) \omega+b_{2}(z) \omega^{2}\right] y(z)
$$

\footnotetext{
${ }^{5}$ Notice that the equation for $y(z)$ is linear and that we can scale $y$ by and arbitrary constant, therefore we can always choose the boundary condition at the horizon to be $y=1$.
} 


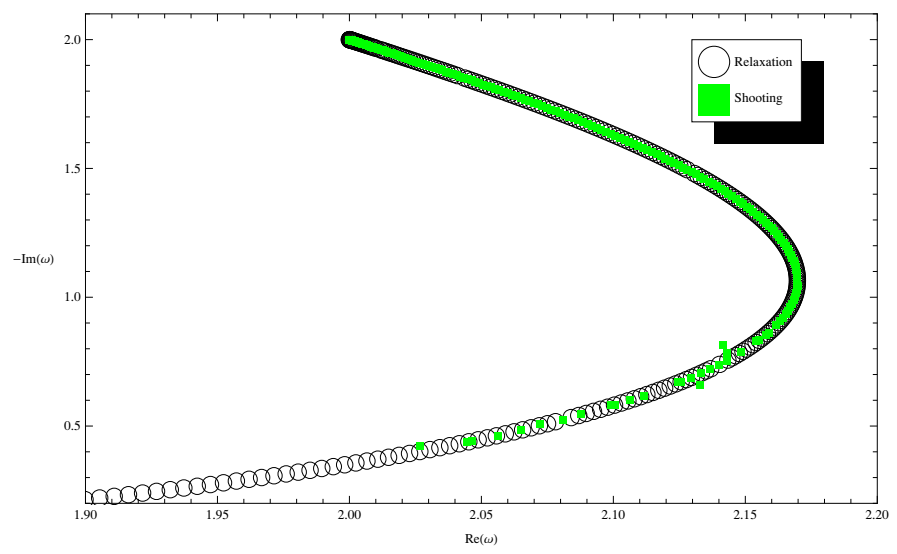

Figure 5. Shoot and relax: comparison of the shooting method result (green squares) with the relaxation method results (black circles) for the location of the first transverse vector quasinormal mode at vanishing momentum $k=0$, density $\tilde{d}=0$. Along the curves the temperature is varied. Our two methods agree very well.
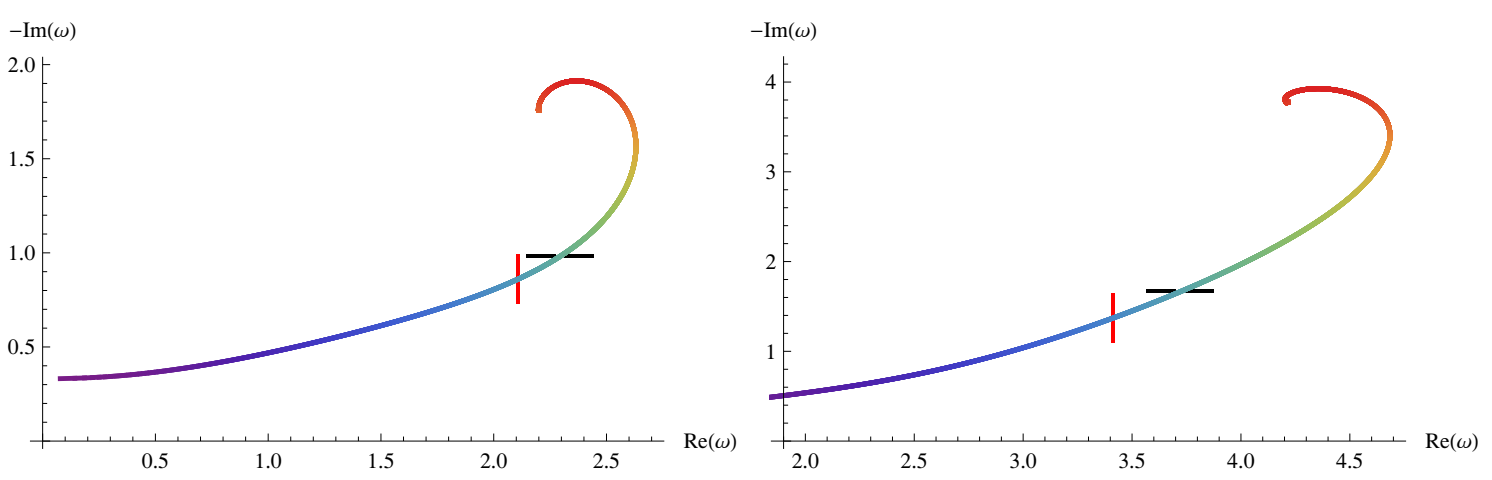

Figure 6. Location of the first and second quasinormal modes in the complex frequency plane for the scalar fluctuations at vanishing momentum $(k=0)$ as a function of the embedding $\chi_{0}$. Red color indicates small quark mass, or high temperature, while the temperature decreases towards blue colors. The horizontal (black) dash indicates the frequency at the phase transition where the angle is $\chi_{0}=0.939$ whereas teh vertical (red) dash indicates the onset of the instability at $\chi_{0}=0.962$.

with

$$
\begin{array}{ll}
a_{1}(z)=A_{1}(z)+\frac{2}{z}, & a_{0}(z)=\frac{A_{1}(z)}{z}+A_{0}(z)-B(z)^{2} k^{2} f(z), \\
c_{1}(z)=\frac{1}{2(1-z)}, & b_{2}(z)=B(z)^{2}-\frac{1}{16(1-z)^{2}}, \\
b_{1}(z)=-\frac{\left(1-A_{1}(z)(1-z)\right) z-2}{4 z(1-z)^{2}} . &
\end{array}
$$

Results for the scalar. The first and second scalar QNM at vanishing momentum can be found in figure 6 . The basic behavior is similar to that of the vector modes. Increasing the quark mass from zero the real part of the QNM frequency again shows a 


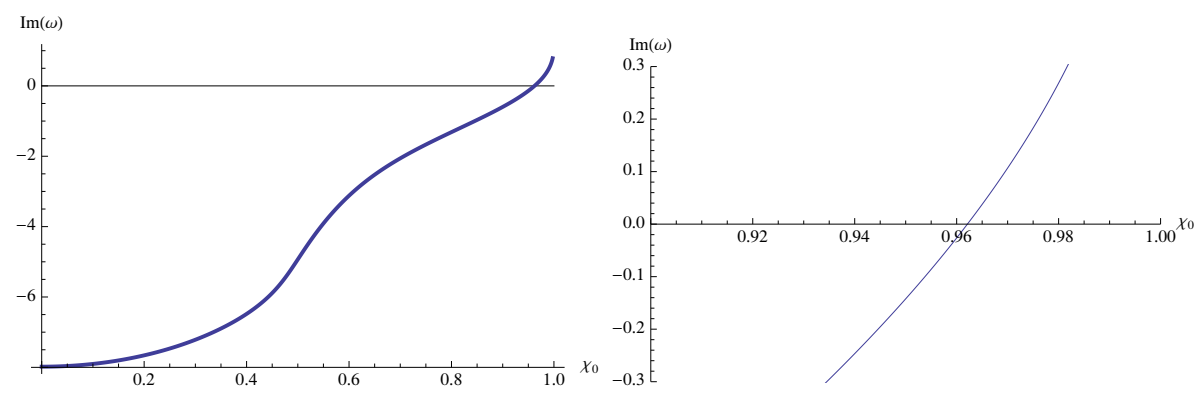

Figure 7. Left: the plot shows a purely imaginary quasinormal mode at $k=0$ as a function of the embedding. Right: zooming into the region where the scalar mode crosses the real axis becoming tachyonic approximately at $\chi_{0}=0.96221$.

turning behavior moving first to larger values, then to smaller values of $\operatorname{Re}(\omega)$. However, in contrast to the vectors, the scalar QNM frequency also shows a turning behavior in the imaginary part $\operatorname{Im}(\omega)$. This means that increasing the quark mass, i.e. decreasing the temperature, the corresponding modes first decay faster, then beyond the turning point they decay slower and slower as the mode approaches the real axis. Moreover the scalar QNMs do not asymptote to the real axis as fast as the vector QNMs do. Instead the scalar QNM frequencies even at large masses still have a considerable imaginary part of roughly 1/2. All the values for the scalar modes are excellent agreement with the ones obtained previously in [13].

The short dash in the figures again shows the location of the known meson melting transition where the initial angle is $\chi_{0}=0.939$. Increasing the mass further while staying in the black hole phase, we observe a scalar QNM to become tachyonic. This point is marked by a vertcal dash in the figures. Figure 7 shows the appearance of an unstable mode explicitely. This particular mode is special since it has vanishing real part but it starts with an extra-ordinary large imaginary part of the QNM frequency at zero quark mass. Increasing the quark mass this purely damped mode moves closer to the real axis until it crosses to become unstable at $\chi_{0}=0.962$ corresponding to the maximal mass for black hole embeddings of $m \approx 1.31$. This particular mode has not been observed in previous studies because at vanishing quark mass it is located very deep in the complex frequency plane near $\operatorname{Im} \omega \approx-8$, while for example the first scalar QNM has $\operatorname{Im} \omega=-2$ at vanishing quark mass. In principle there could be an infinite tower of such purely imaginary modes, each crossing the real axis at the larger and larger quark mass. However, the accuracy of our numerics proved insufficient to establish additional modes beyond this lowest one. In any case once this mode has crossed the real axis the D7 brane embedding is locally unstable and can not be taken as a (metastable) ground state. This raises the question of what is the true ground state in this regime. It might be that there is another type of D7brane embedding that is reached somehow by condensation of the scalar mode. Another possibility is that there is simply no locally stable embedding beyond that point. 


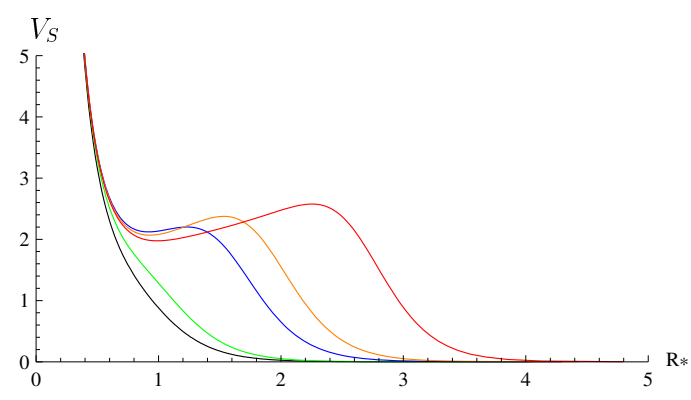

(a)

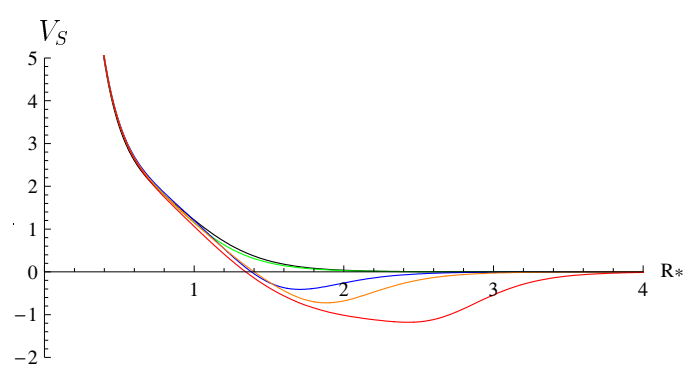

(b)

Figure 8. Schrödinger potential of the vector (a) and scalar (b) fluctuations for different values of $\chi_{0}$. The different colors correspond to $\chi_{0}=0$ (black), 0.5 (green), 0.9 (blue), 0.95 (orange), 0.99 (red).

\subsection{Schrödinger potential analysis}

In this section we present a qualitative analysis of the quasinormal spectrum using the fact that the equations of motion for the fluctuations can be rewritten in the form of the Schrödinger equation (see appendix $\mathrm{C}$ for more details)

$$
-\partial_{R *}^{2} \psi+V_{S} \psi=E \psi
$$

where $R *$ is a tortoise-like coordinate. The Schrödinger potential $V_{S}$ determines the energy spectrum $E$ which is related to the quasinormal spectrum by $E=\omega^{2}$.

At zero baryon density and zero momentum, the potentials for the vector and scalar modes are already discussed in [25] and [14], respectively. Nevertheless we include the discussion here for completeness. In figure 8 we present the Schrödinger potential for the vector and scalar fluctuations at different quark masses parametrized by $\chi_{0}$. In these plots we observe an infinite wall in the potential at $R *=0$ which corresponds to the AdS boundary. In addition to this wall the potential for the vector modes develops a stepshape as we increase the quark mass. In [25] it is shown that the imaginary part of the quasinormal frequency decreases as the step gets longer which is consistent with our result found in figure 4 .

For the scalar modes a negative well arises in the Schrödinger potential. This well becomes deeper and wider as we increase the quark mass and therefore support a 'bound' state with $E<0$ which corresponds to a tachyonic quasinormal frequency $\operatorname{Im} \omega>0$. This well and the 'bound' state are studied in [14]. The Schrödinger analysis clearly shows the existence of a tachyonic mode which we already found in figure 7.

\subsection{Discussion: tachyon and de-singularization}

In this section we discuss why the so-called undercooled phase shows unphysical meson spectra which do not approach the known ones in the supersymmetric limit. Further we discuss that finite density cures this behavior by de-singularizing the geometry, i.e. by smoothing out the limiting embedding. 
As mentioned above the scalar fluctuation becomes tachyonic once the quark mass parameter has reached its maximum as a function of $\chi_{0}$. It is not to be expected that the region beyond that point contains physically relevant or meaningful signatures. This region contains the limiting embedding which only touches the horizon and geometrically separates Minkowski from black hole embeddings. Here meson spectra had been studied earlier [14, 25]. These meson spectra display a singular behavior in the sense that all the quasinormal modes (first, second, ... ) approach one single 'attractor' energy (or frequency) near the limiting embedding. The geometric reason for this is the scaling symmetry for the embeddings in the near-critical region [12]. That scaling symmetry implies that near the critical quark mass (or temperature) there exists no preferred scale on the brane. In this sense there is no scale which could determine the distance between resonances in the brane fluctuations, i.e. between the distinct meson mass resonances, or quasinormal modes equivalently.

At finite fixed baryon density however, this particular scaling symmetry is broken [16]. Therefore the chemical potential introduced together with that density does set the scale for the separation between the distinct quasinormal modes. This is somewhat analogous to the behavior of the thermal quasinormal mode spectrum when the temperature of the dual black hole background is lowered towards zero. In that case the quasinormal modes are known to approach one single value, i.e. the spectrum becomes singular in our sense. There the scale which determines the distance between distinct excitations is clearly the temperature since it is the only scale in the theory. In the zero temperature limit this scale vanishes and the scaling symmetry is restored.

As observed earlier [30] the meson spectrum at finite density approaches the supersymmetric one at low temperatures. In this sense the theory is de-singularized by finite baryon density. We will see this explicitly in the quasinormal mode spectra at finite density in section 6 .

\section{Finite momentum but vanishing density}

We now turn to the case of non-zero spatial momentum.

\subsection{Transverse vectors}

We obtain results at finite momentum from the numerical solution of equation (4.1) with distinct non-zero values of $k$.

Figure 9 shows the first of the transverse vector QNMs at different values of the spatial momentum $k$. The behavior in the region $k=0, \ldots, 4$ is very similar to the $k=0$ case. Although its trajectory in the complex frequency plane becomes more wavy at larger $k$, the first scalar QNM still starts at quite large real and imaginary parts in order to approach the real axis and smaller real parts when temperature is decreased. Distinct curves for different values of $k$ within numerical accuracy approach a single limiting value $\omega_{0}$ at small temperatures. It is interesting to note that the turning point in $\operatorname{Re}(\omega)$ mentioned in the previous section for $k=0$ disappears when $k$ reaches values between $k=1$ and $k=2$. 

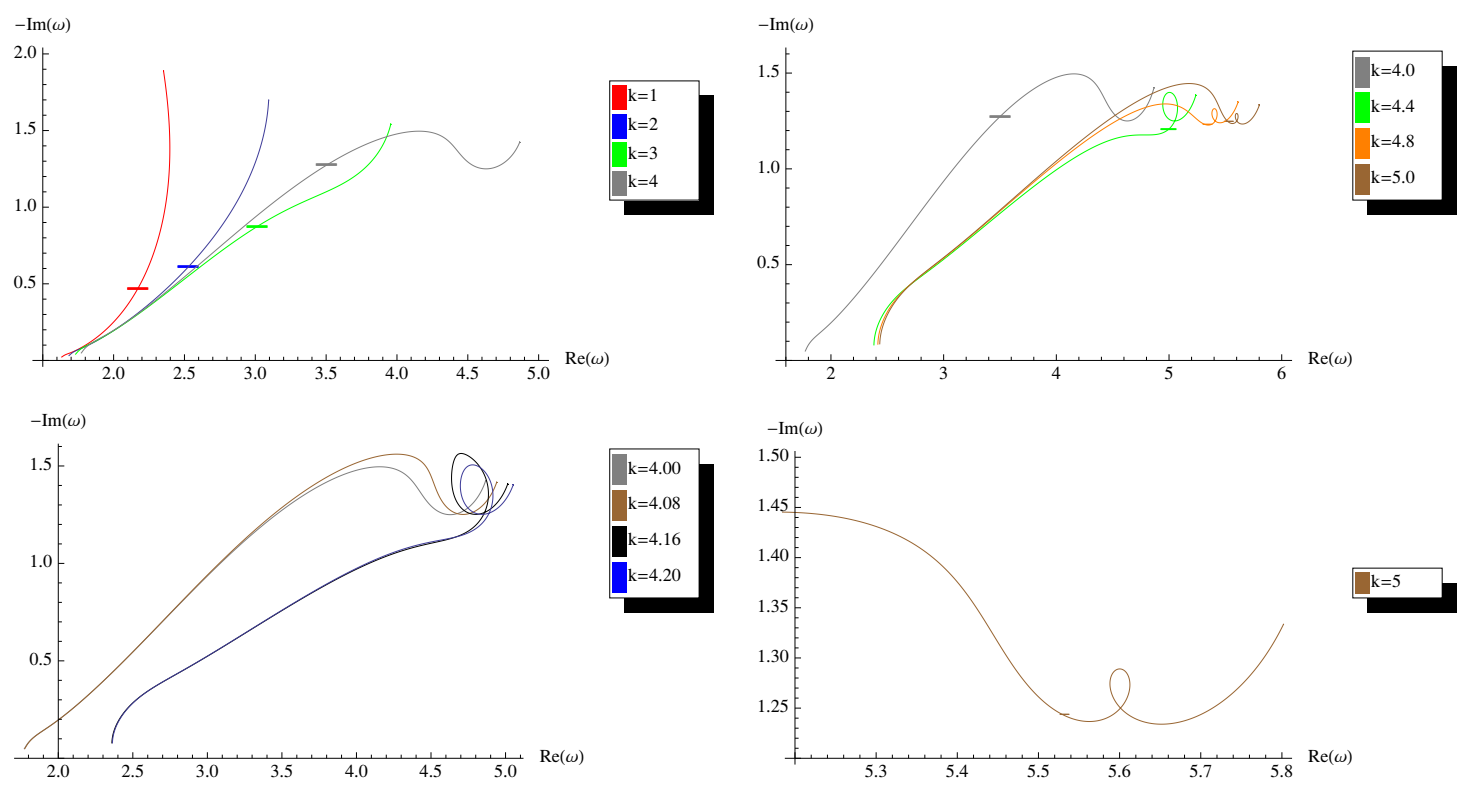

Figure 9. First quasinormal mode for transverse vector fluctuations at different spatial momenta $k$. Horizontal short dashes across the curves show the location of the meson melting transition. At $k \approx 4.16$ a spiral structure appears and the curves also asymptote to a distinct small temperature value from that $k$ on. Along the curves the quark mass parameter $\chi_{0}=\cos \Theta_{0}$ is varied. See figure 2 for its relation to the quark mass and temperature.

A quite substantial qualitative change appears at $k=4.16$, where the trajectory of the mode in the frequency plane develops one loop. Also the trajectories at higher $k$ have this looping behavior. At the same time these curves with one loop do asymptote to a single small temperature frequency value $\omega_{1}$ as well. But this limiting value is distinct from the limiting value which is approached by the low $k$ curves without the loop, i.e. $\omega_{1} \neq \omega_{0}$. This fact suggests that the loop-behavior and the distinct limiting value are somehow related. For the longitudinal vector fluctuations (see figure 11) we will explicitly see that this relation generalizes to all fluctuations and to higher loops in this way: all the first QNM trajectories for scalar and vector fluctuations with $k<k_{n}$ have $n$ loops and they asymptote to a small temperature limit frequency $\omega_{n}$ (within numerical accuracy) with $\omega_{n+1}>\omega_{n}$. Note that the loop behavior appears in a physical, thermodynamically preferred phase, i.e. before the meson melting transition and also way before the tachyon appears.

The loops are absent in the second quasinormal mode as seen from figure 35. Nevertheless the second quasinormal mode also asymptotes to distinct low temperature limits $\omega_{n}$ above distinct certain momenta $k_{n}$.

Figure 10 captures the dispersion relation of the first transverse vector QNM at different values of $\chi_{0}$. 

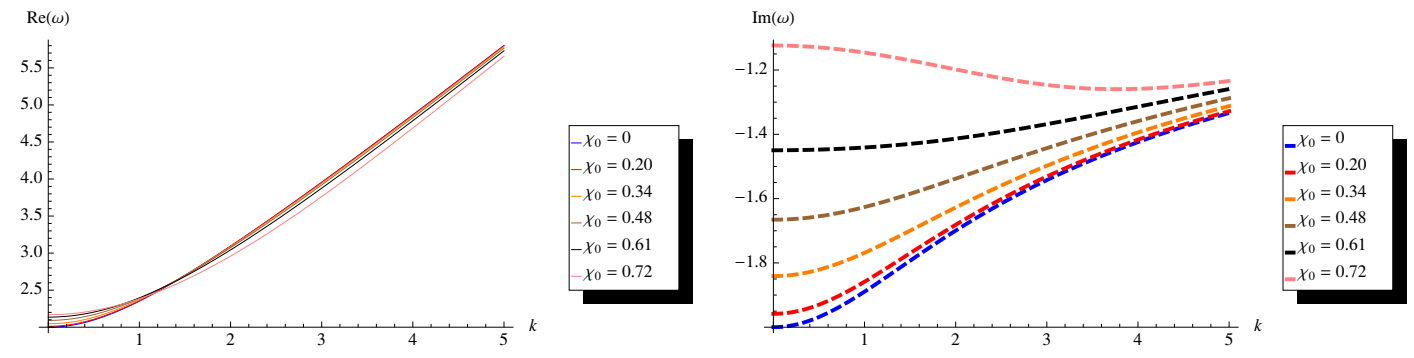

Figure 10. Dispersion relation for the first transverse quasinormal mode at distinct quark masses, or equivalently temperatures, parametrized by the embedding parameter at the horizon, $\chi_{0}$. See figure 2 for the relation between $\chi_{0}$, the temperature and quark mass $M_{q}$.
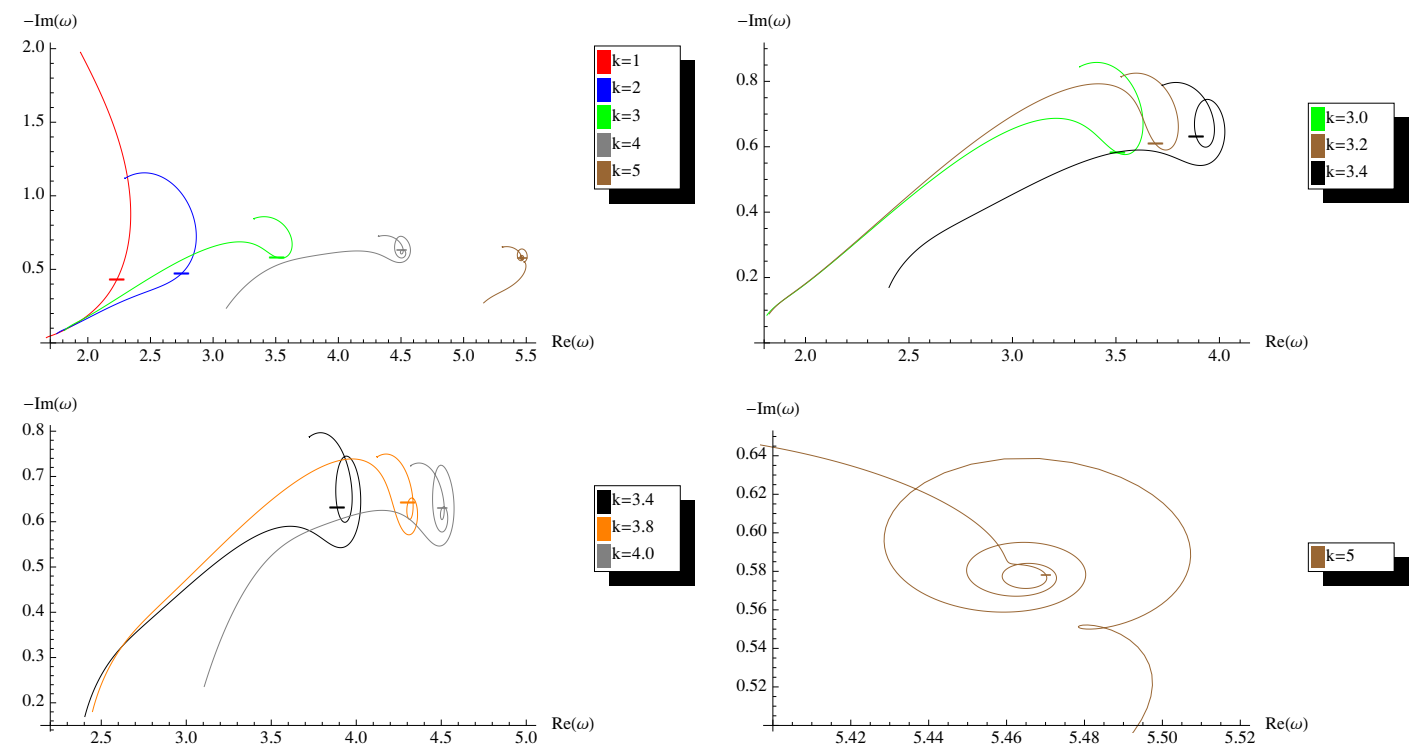

Figure 11. The first quasinormal mode for longitudinal vector fluctuations at distinct values of k. Along the curves the quark mass parameter $\chi_{0}=\cos \Theta_{0}$ is varied. See figure 2 for its relation to the quark mass and temperature.

\subsection{Longitudinal vectors}

We obtain results at finite momentum from the numerical solution of equation (4.5) with distinct non-zero values of $k$.

The behavior of the longitudinal vector QNMs is qualitatively similar to that of the transverse ones discussed in section 5.1. The mentioned loops in the frequency plane trajectory do appear at smaller values $k \approx 3.4$ in the longitudinal channel than they do in the transverse one. However, while in the transverse vector case the loops appeared before the meson melting transition, in the longitudinal case the transition takes place before the first loop is terminated as can be seen from the figure 11. Just as for the transverse vectors, 

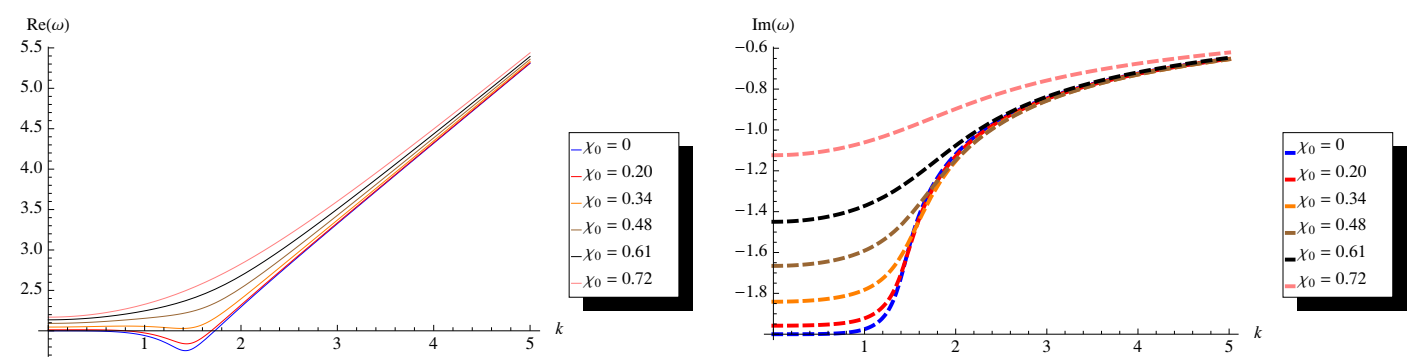

Figure 12. Dispersion relation for the first quasinormal longitudinal vector mode fluctuation at distinct values of the quark mass parameter $\chi_{0}$ (see figure 2 for its relation to the quark mass at vanishing density).

also the second QNM of the longitudinal vectors does not have any loops in its complex frequency plane trajectory, as figure 37 shows.

The dispersion relations for the first and second QNM of the longitudinal vector fluctuation are depicted in figure 12 and figure 38 in appendix E, respectively.

\subsection{Scalar}

We obtain results at finite momentum from the numerical solution of equation (4.7) with distinct non-zero values of $k$.

The general behavior of the scalar QNMs is qualitatively similar to that of the longitudinal vector QNMs. Figure 13 shows the first of the scalar QNMs at momenta between $k=1$ and $k=5$. Also in this case the overall behavior is that the real and imaginary parts decrease as temperature is decreased along the curves. At small $k$, e.g. $k=1$, there is a turning point present in the real as well as in the imaginary part. These turning points again disappear between $k=1$ and $k=3$. Just like for the first of the longitudinal vector QNMs multiple loops form successively for larger values of $k$. The meson melting transition appears before the first of the loops has terminated. Again the number of loops seems to be directly related to the low temperature value $\omega_{n}$ to which the curves for all $k>k_{n}$ asymptote. Dispersion relations for the first scalar QNM are shown in figure 14 . The corresponding figures for the second scalar QNM are figure 39 and 40 in appendix E. Just like for the vectors there are also no loops in the second QNM for the scalars.

\subsection{Schrödinger potential analysis}

Just as in the previous section at zero density and momentum, we here compute the effective potential for the scalar and vector fluctuation equations (4.7) and (4.5), (4.1) (see appendix $\mathrm{C}$ for more details).

We begin by examining the Schrödinger potential for the scalar fluctuations in figure 15 . The lowest (red) curve shows the potential at vanishing momentum and density at $\chi_{0}=$ 0.9999. That is near the limiting embedding, far beyond the thermodynamic transition to Minkowski embeddings and far beyond the appearance of the tachyon in the spectrum. The 

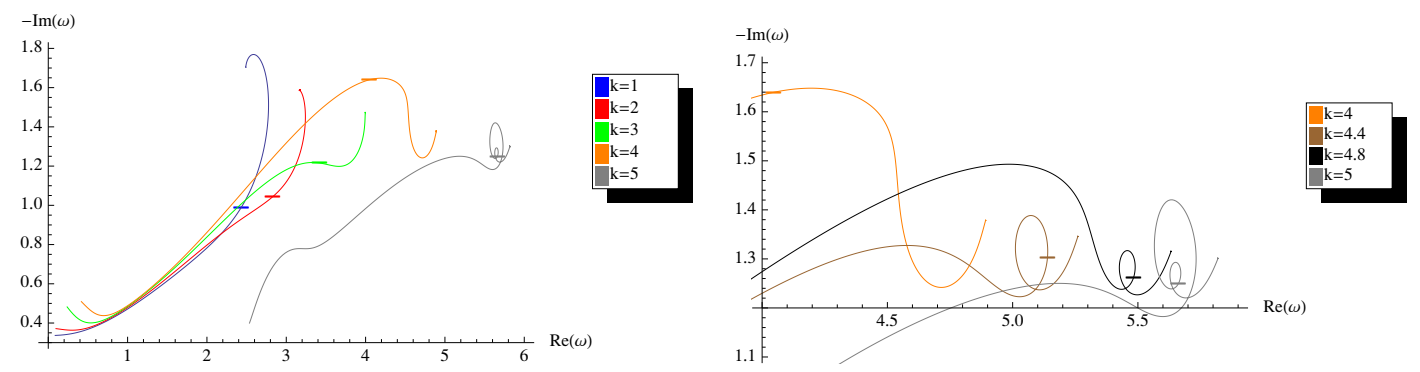

Figure 13. First scalar quasinormal mode at distinct momenta $k$. Along the curves the quark mass parameter $\chi_{0}=\cos \Theta_{0}$ is varied. See figure 2 for its relation to the quark mass and temperature.
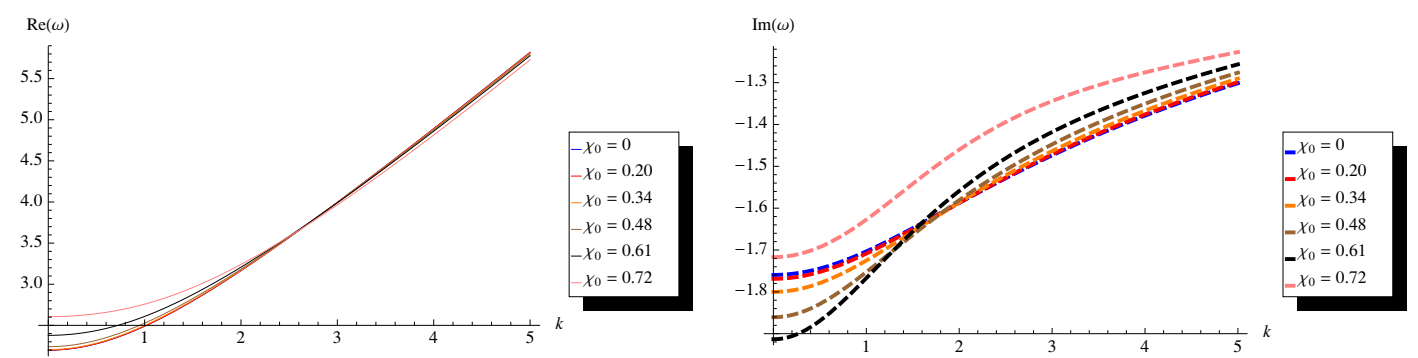

Figure 14. Dispersion relation for the first scalar quasinormal mode at distinct values for the quark mass parameter $\chi_{0}$ (see figure 2 for its relation to the quark mass at vanishing density). The real part of the QNM frequency is shown on the left, the imaginary part on the right.

results at smaller $\chi_{0}$ are qualitatively the same, but we plot large $\chi_{0}$ in order to investigate the tachyon and the reason for the different "attractor" frequencies in that large mass regime. The scalar potential clearly exhibits a wide negative dip in which the tachyonic scalar mode resides, compare figure 7. As the momentum is increased the potential is lifted and the negative dip is narrowed. In this way the lowest possible excitation is pushed towards more positive energy values becoming non-tachyonic at large momenta. However the theory is clearly unstable against condensation of the scalar fluctuations already at $\chi_{0} \geq 0.962$ and $k=0$. In figure 16 we zoom out to larger values of the potential. For increasing momentum, a step forms near the boundary. When comparing to figure 8 (a) we see that the scalar potential at finite momentum is similar to the vector case at zero momentum. In figure 16 the step becomes higher and longer for increasing momentum, while its plateau becomes shorter, i.e. most of the plateau is located near the boundary. Therefore conceptually the analysis of [25] as discussed in section 4.4 applies as in the vector case: when the step becomes longer, the imaginary part of the quasinormal frequencies decreases. This is consistent with our observations in figure 13 (compare for example the initial points where $\chi_{0}=0$ for increasing momenta from curve to curve). The increasing real part of the quasinormal frequency observed in figure 13 is due to the fact that the 


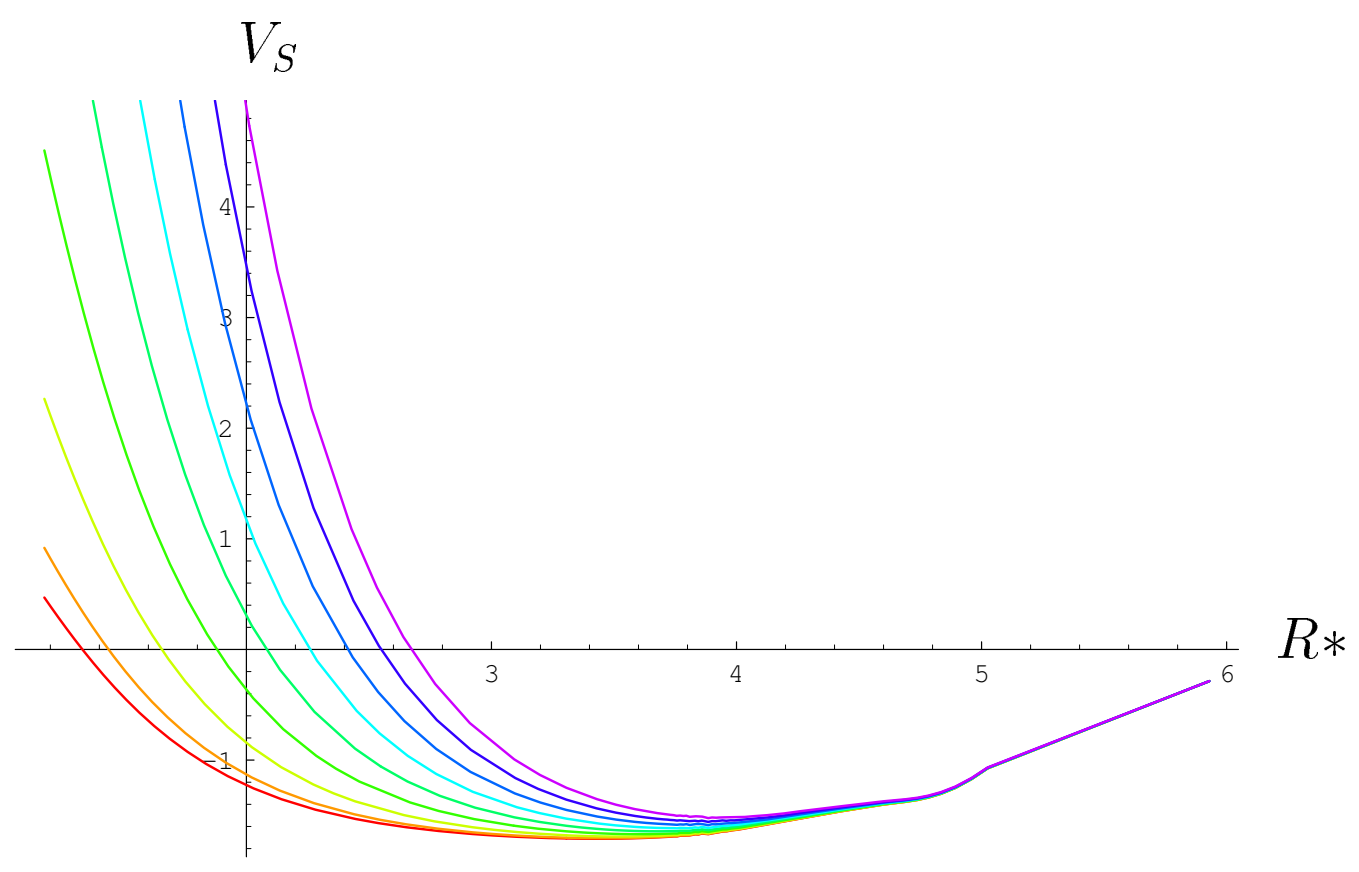

Figure 15. The scalar Schrödinger potential $V_{s}$ (zooming in on the minimum of the potential) versus the radial coordinate $R *$ defined in section 4.4 at increasing momenta $k=$ $0,2,4,6,8,10,12,14,16$ from bottom to top curve with the quark mass parameter $\chi_{0}=0.9999$ (see figure 2 for its relation to the quark mass at vanishing density), $\tilde{d}=0$. The dip supporting the tachyon narrows.

potential step rises closer to the horizon at larger momentum. This narrows the potential and rises the excitation energies.

Turning now to the transverse vector fluctuations, we observe a step potential in figure 17. The larger the momentum, the earlier the potential rises towards infinity when approaching the boundary at $R *=0$. So effectively the boundary moves towards the horizon and the length of the plateau of the potential step becomes shorter. This is different from the scalar modes discussed above. While the imaginary part of the quasinormal frequency decreases for increasing momentum just as in the scalar case, the Schrödinger potential shows a different behavior: for increasing momentum, the Schrödinger potential approaches the shape of a wall. Where as in the scalar case, the formation of the step is responsible for lowering the imaginary part of the quasinormal frequency, here we expect that the formation of the wall is responsible for a similar decrease of the imaginary part of the quasinormal frequency.

\subsection{Discussion: breakdown of hydrodynamics, 'attractors' and the tachyon}

In this discussion we focus on three distinct physical implications of the quasinormal modes described previously in this section. The longitudinal vector modes tell us when the hydrodynamic approximation breaks down, while the novel purely imaginary scalar mode renders the whole theory unstable as it becomes tachyonic. Both scalar and vector modes asymp- 


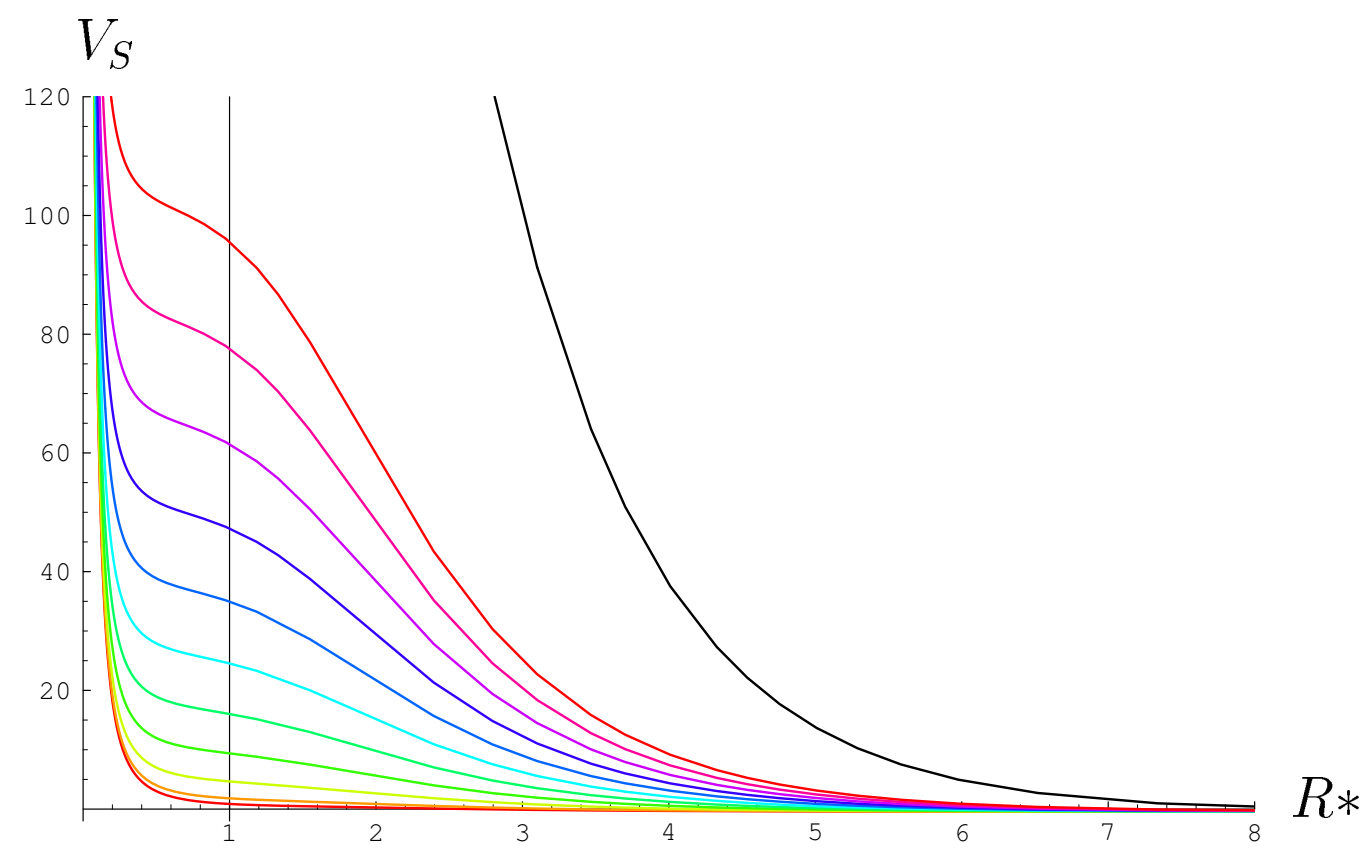

Figure 16. The scalar Schrödinger potential $V_{s}$ (zooming out to larger values of the potential) versus the radial coordinate $R *$ at increasing momenta $k=0,2,4,6,8,10,12,14,16,18,20,40$ from bottom to top curve with quark mass parameter $\chi_{0}=0.9999$ (see figure 2 for its relation to the quark mass at vanishing density), $\tilde{d}=0$. A step forms towards the boundary, similar as that for the vectors. The grid line at $R *=1$ serves to guide the eye only.

tote to 'attractor' frequencies. This behavior is probably related to a spiraling behavior of the quasinormal mode's trajectories with changing quark mass or temperature.

\subsubsection{Hydrodynamics to collisionless crossover}

Contrary to the transverse vector and scalar channel the longitudinal vector channel also has a hydrodynamic quasinormal mode, i.e. a mode whose dispersion relation does not show a gap at zero momentum, $\lim _{k \rightarrow \omega} \omega(k)=0$ (see figure 18, left). This mode represents diffusion of baryon charge. It is a mode whose frequency is purely imaginary and therefore results in a purely damped time evolution without any oscillation. At small momentum the dispersion relation is well approximated by the diffusion kernel $\omega=-i D k^{2}$, as seen in figure 18 on the left. Fitting our numerical data to this we can extract the diffusion constant $D$. Since it has been calculated before in the literature in [14] we do not restate this result. We checked however that our values are consistent with the results there.

On general grounds one expects that a many body system shows a crossover from hydrodynamic behavior at long wavelengths to a coherent or collisionless behaviour at small wavelengths. In the holographic context this has first been discussed in an $A d S_{4}$ example in [31] by calculating spectral functions. A more direct way to see this crossover can be obtained by studying the quasinormal mode spectrum. At small momentum the hydrodynamic mode should be the dominant one, i.e. the one with the largest imaginary part. At small wavelength we expect the dominant modes to have frequencies whose imaginary part 


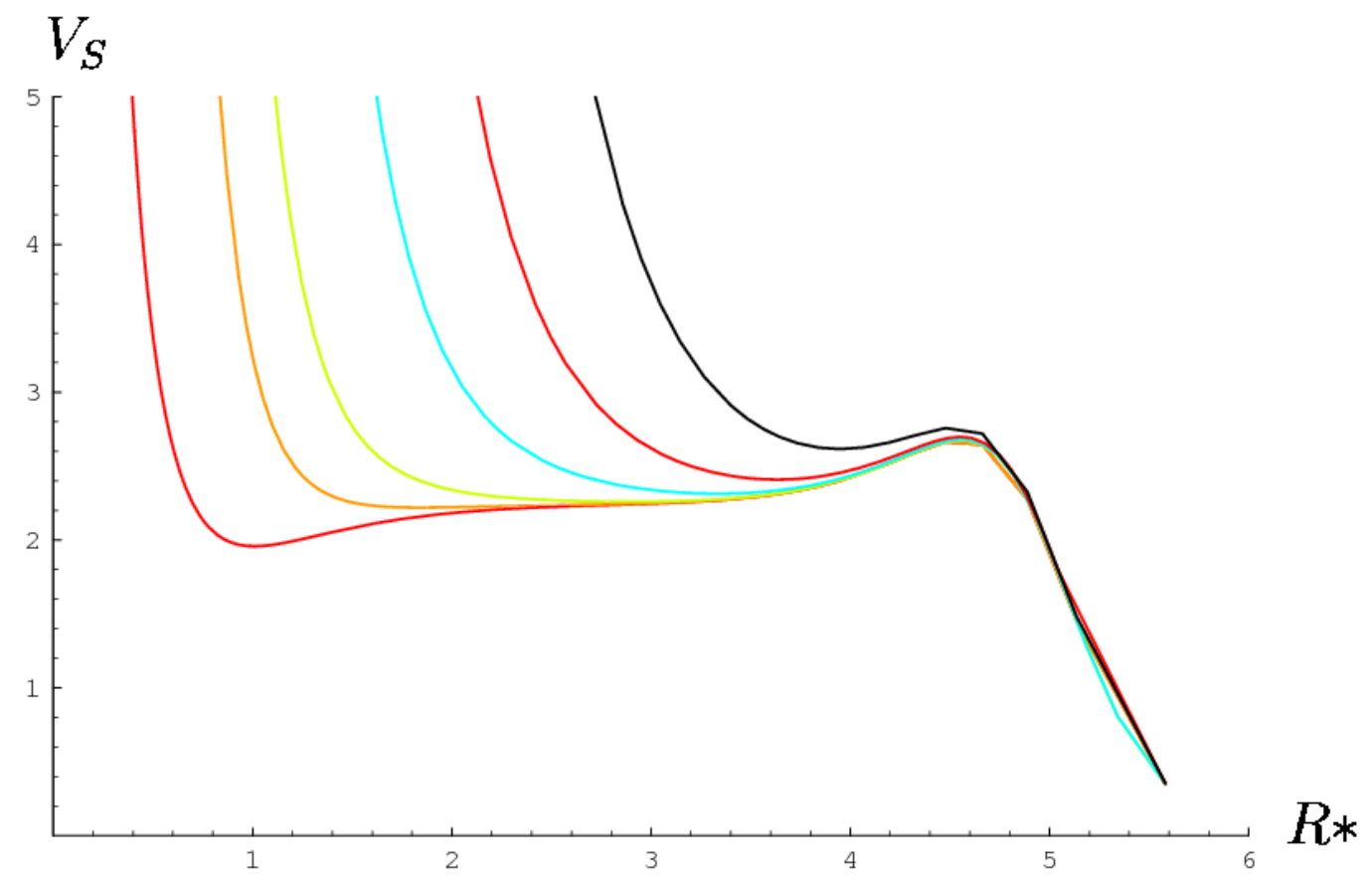

Figure 17. The transverse vector Schrödinger potential $V_{s}$ versus the radial coordinate $R *$ at increasing momenta $k=0,2,4,10,20,40$ from bottom to top curve with quark mass parameter $\chi_{0}=0.9999$ (see figure 2 for its relation to the quark mass at vanishing density), $\tilde{d}=0$. The potential step becomes shorter since the potential rises farther and farther away from the boundary $R *=0$.

is much smaller than their real parts. This means that at large wavelength the response would be simply an exponential decay whereas at short wavelength the response would be a slowly decaying oscillation. In terms of the quasinormal mode spectrum this implies that the purely imaginary diffusion mode as a function of momentum has to cross the imaginary part of the dispersion relation for the lower non hydrodynamic modes. Indeed this is what happens for the R-charge and momentum diffusion in the strongly coupled $\mathcal{N}=4$ theory as discussed in $[27,28]$. We therefore define the crossover from the hydrodynamic regime to the collisionless regime through the momentum at which the imaginary part of the lowest non-hydrodynamic mode crosses the purely imaginary diffusion mode. From that wavelength on it is the lowest gaped quasinormal mode which dominates the late time response. ${ }^{6}$ It should be mentioned that there is at least one other way of how this crossover

\footnotetext{
${ }^{6}$ There is a small puzzle related to that. If the prolongation of the hydrodynamic mode to large momenta is constantly increasing, the front velocity computed from it seems to violate causality. As is well-known already the diffusion kernel violates causality because of the $k^{2}$ behaviour and the extension to larger momenta shows even higher exponents in the dependence on $k$. So how manages the theory to preserve causality? The resolution has been presented in [27]: the residues of the diffusive quasinormal mode vanish for large momenta and therefore this mode ceases to exist in the dangerous limit of large $k$. On the other hand one can study the hydrodynamics by fixing a real frequency and then looking for complex roots in the momentum $k$ as it has been done in [32]. The quasinormal frequencies or the complex momenta respectively are roots of infinite order polynomials (or at least of extremely high order polynomials in a truncated approximation of the holographic Green function). Therefore it is not possible to simple infer
} 

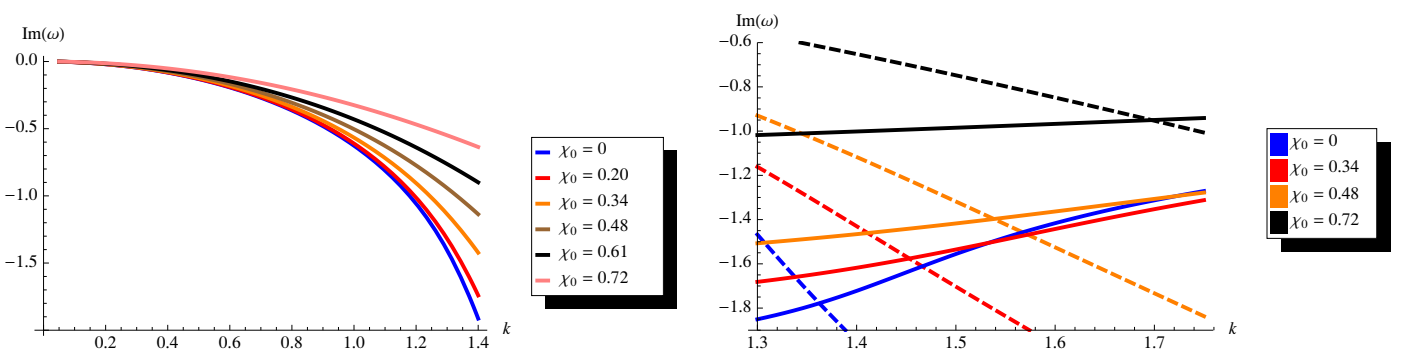

Figure 18. Left: the dispersion relation for the diffusion mode at distinct values for the mass/temperature parameter $\chi_{0}$ (see figure 2 for its relation to the quark mass at vanishing density). Right: intersection of the diffusive mode with the imaginary part of the first longitudinal quasinormal mode. The intersection point moves to larger values of $k$, but to smaller values of $\operatorname{Im}(\omega)$ as the mass/temperature parameter $\chi_{0}$ is increased.

can be established in terms of quasinormal modes. It could also be that the purely imaginary diffusion mode pairs up with another purely imaginary but non-hydrodynamic mode which allows them to develop real parts as well and to move off the imaginary axis. This seems to be the preferred mechanism for $A d S_{4}$ black holes [33] and it also appears on probe D-branes representing defects in the four-dimensional strongly coupled CFT [34]. As will be shown in a companion publication this is also what happens if finite baryon density is introduced [35]. This crossover has recently also been investigated for AdS black holes in various dimension in the time domain in [36].

We have numerically determined the crossover point defined above for different embeddings. As seen from the right side of figure 18, the crossover moves to higher momenta as the embedding angle $\chi_{0}$ is increased. This means that the brane responds to baryon charge fluctuations in a purely absorptive way for smaller and smaller wavelengths as the quark mass is increased, or equivalently as the temperature is decreased. Of course the actual rate of absorption given by the absolute value of the imaginary part of the frequencies decreases with decreasing temperature. Nevertheless it is somewhat surprising that the crossover towards the collisionless regime takes place at smaller wavelengths for lower temperatures.

\subsection{2 "Attractor" frequencies}

Here we briefly discuss the appearance of spiraling QNM trajectories and their relation to the "attractor" frequencies found in section 5.

First we should note that in most cases the spirals in the QNM trajectories occur before the scalar in the spectrum becomes tachyonic. Thus the spiral is a physical signature on the stable or metastable branch of the theory. However the "attractor" frequencies to which the trajectories asymptote at large quark mass parameters $\chi_{0} \rightarrow 1$ are located deep in the

the behaviour of the complex momentum modes from the quasinormal modes. Indeed as shown in [32] the front velocity of hydrodynamic mode as calculated from the complex momentum roots behaves perfectly causal and approaches 1 to very good numerical accuracy. 
unstable phase of the theory. Therefore the "attractor" frequency is no signature of the physical stable sector of the theory. Nevertheless it would be interesting to understand the apparent direct relation of these "attractor" frequencies and the number of spirals in the QNM trajectories since the spirals are physical, as noted before.

Note that a spiraling behavior for changing the temperature has been observed in the quark condensate in this system for near-limiting brane embeddings in [12]. There the spirals are due to oscillations of the parameters of the embedding, i.e. the quark mass and the quark condensate. In particular the asymptotic value $m$ oscillates. This behavior has only been observed in the near-limiting embeddings. In contrast to that our loops in the QNM-trajectories appear way above the critical embedding already. Nevertheless, as stated before there is an apparent connection between the number of loops in our QNMtrajectories and the near-limiting 'attractor' frequency. In this way we could argue that at finite momentum we see the near-limiting embedding oscillations reflected already in the non-critical region in spiraling QNM-trajectories. In other words both the QNM-loops and the spiraling quark condensate might have the same origin, namely the oscillating embedding parameters which are related to the aforementioned scaling symmetry of the near-limiting embedding [12].

Unfortunately the Schrödinger potentials at finite momentum but vanishing density close to $\chi_{0}=1$ in figure 15 and 17 do not show any distinct feature hinting neither on discrete special frequencies $\omega_{n}$ nor on the jumps between them at critical momenta.

\subsubsection{Tachyon: a new hydrodynamic mode}

We briefly discuss here the behavior of the scalar mode becoming tachyonic as explained in the previous section. This mode turns into a hydrodynamic mode in a special case.

As expected this scalar mode becomes tachyonic at higher and higher values for the quark mass parameter $\chi_{0}$ as the momentum of the excitation is increased. In the parameter space spanned by charge density, temperature and quark mass there is one interesting special point: that is the location $\chi_{0}^{\text {crit }}(\tilde{d})$ where the scalar mode becomes tachyonic. Just at this special quark mass/temperature value, this scalar mode develops a hydrodynamic dispersion relation, i.e. $\lim _{k \rightarrow 0} \omega \rightarrow 0$. In other words the scalar mode which shows the instability of the system turns into a hydrodynamic mode just at the critical point. This could signal that there is a transition to a new phase. For instance this transition might be similar to the glass transition in supercooled liquids discussed e.g. in [37]. Lastly there might not exist a new stable ground state since we might be scanning a regime where no stable brane embedding exists besides the thermodynamically preferred Minkowski embedding.

\section{Finite density but vanishing momentum}

In this section we turn back to zero momentum, but switch on a finite baryon density and chemical potential. The non-normalizable mode of the zero component of the gauge field living on the D7-brane gives the chemical potential in the field theory. Working in the $\rho$ coordinates we define

$$
\lim _{\rho \rightarrow \infty} A_{0}(\rho)=\mu
$$


In order to study vector mesons in this background we consider fluctuations of the gauge field about this background field. The equation of motion for the zero component of the gauge field thus reads [16]

$$
\partial_{\rho} A_{0}=2 \tilde{d} \frac{f \sqrt{1-\chi^{2}+\rho^{2} \chi^{\prime 2}}}{\sqrt{\tilde{f}\left(1-\chi^{2}\right)\left[\rho^{6} \tilde{f}^{3}\left(1-\chi^{2}\right)^{3}+8 \tilde{d}^{2}\right]}} .
$$

Since the asymptotic behavior for the embedding function $\chi$ is known we can also give the asymptotics for the gauge field at infinity

$$
A_{0}=\mu-\frac{1}{\rho^{2}} \frac{\tilde{d}}{2 \pi \alpha^{\prime}}+\ldots,
$$

where $\mu$ is, as already mentioned, the chemical potential and $\tilde{d}$ parametrizes the baryon number density $n_{B}$ by

$$
\tilde{d}=\frac{2^{\frac{5}{2}} n_{B}}{N_{f} \sqrt{\lambda} T^{3}}
$$

We divided this section into four sections in the same manner as the former ones, except that we do not have to handle longitudinal vectors here since $k=0$. But we will investigate the transverse vectors and the scalar modes.

Then we will describe our results, especially the tachyon behavior and the turning points of the meson mass. Furthermore we present a Schrödinger potential analysis to understand the above results in a different way. We investigate the turning behavior of the quasinormal frequencies further by an analytical calculation close to the horizon at large frequencies.

\subsection{Transverse vectors}

To obtain the equation of motion for the transverse vector modes, we have to vary the DBI action with respect to the gauge field fluctuations about the background and retain these terms up to second order [30]. The equation of motion for the fluctuations of the gauge field reads

$$
0=E^{\prime \prime}+\partial_{\rho} \log \left(\frac{f}{\tilde{f}} \sqrt{1-\chi^{2}} \sqrt{\frac{\rho^{6} \tilde{f}^{3}\left(1-\chi^{2}\right)^{3}+8 \tilde{d}^{2}}{\tilde{f}\left(1-\chi^{2}+\rho^{2} \chi^{\prime 2}\right)}}\right) E^{\prime}+2 \omega^{2} \frac{\tilde{f}}{f^{2}} \frac{1-\chi^{2}+\rho^{2} \chi^{\prime 2}}{\rho^{4}\left(1-\chi^{2}\right)} E
$$

The connection between the gauge invariant field $E$ and the gauge field is simply $E=\omega_{p h} A_{i}$, where we are free to choose any Minkowski spatial direction $i=1,2,3$. Furthermore the parameter $\omega$ is a dimensionless frequency defined as $\omega=\omega_{p h} / \pi T$ with the physical frequency $\omega_{p h}$. A more detailed derivation of this equation of motion can be found in [30].

The solution to this equation of motion can be obtained by numerical methods, described in appendix A. Then we can compute the correlator in the complex plane where the quasinormal modes appear as poles. We aim for an explanation of the turning behavior 


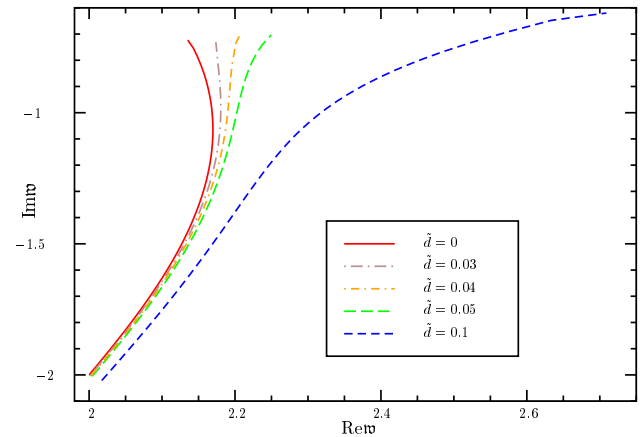

(a)

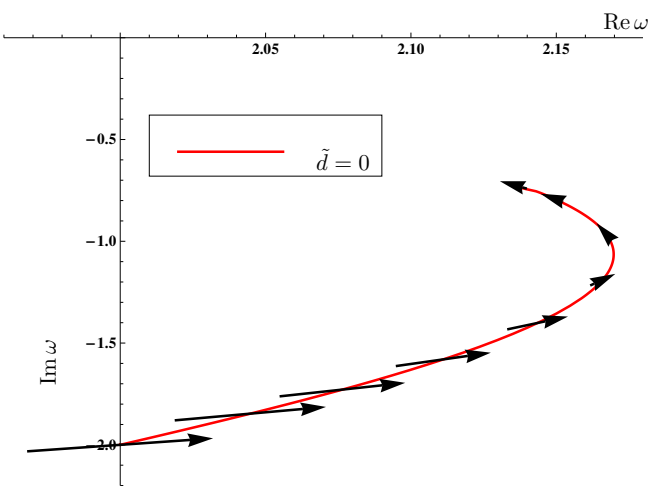

(b)

Figure 19. (a) First quasinormal modes for several densities $\tilde{d}$. For smaller densities a turning point in the real part occurs, which vanishes as the densities rises. (b) The first quasinormal mode for vanishing density. The arrows display the residue of the pole at the specific point, particularly the direction its phase and the length its absolute value.

of the meson mass found in [30]:

at large quark mass the meson mass increases proportional to the quark mass $M_{q}$ as expected from the formula found in the supersymmetric case for vector and scalar meson masses (for vanishing angular momentum $l=0$ on the $S^{3}$ ) [8]

$$
M_{\text {meson }}=\frac{2 M_{q}}{\sqrt{\lambda} T R^{2}} \sqrt{2(n+1)(n+2)}, \quad n \in \mathbb{N} .
$$

Whereas for small quark mass there exists a region where the meson mass is decreasing when the quark mass is raised.

Results for transverse vectors. Figure 19 (a) displays the paths of the first quasinormal mode in the complex frequency plane for different densities. The paths are parametrized by the quark mass over temperature ratio. When the quark mass is zero, i.e. our embedding is flat, the quasinormal mode is located near the point 2(1-i) and moves towards the real axis as the quark mass increases. For small densities we see that the curves turn around and tend towards smaller real values for larger quark mass. This behavior disappears when we raise the density up to a critical density $\tilde{d}_{c} \approx 0.04$.

\subsection{Scalar}

In order to compute the spectral function for scalar modes we first calculate the pullback of the metric to the D7-branes and expand in the fluctuations of the embedding variables $\delta \Theta$ and $\delta \phi . \delta \Theta$ corresponds to the scalar and $\delta \phi$ to the pseudoscalar excitations. We consider time and $\rho$ dependence of the fluctuations only, since we stay at zero momentum. The induced metric then reads 


$$
\begin{aligned}
\mathrm{d} s^{2}= & \frac{1}{2} \frac{\rho^{2}}{R^{2}}\left(-\frac{f^{2}}{\tilde{f}} \mathrm{~d} t^{2}+\tilde{f} \mathrm{~d} x_{i}^{2}\right)+\frac{R^{2}}{\rho^{2}} \frac{1-\chi^{2}+\rho^{2} \chi^{\prime 2}}{1-\chi^{2}} \mathrm{~d} \rho^{2}+R^{2} \sin (\Theta+\delta \Theta) \mathrm{d} \Omega_{3} \\
& -2 R^{2} \frac{\chi^{\prime}}{\sqrt{1-\chi^{2}}} \partial_{a} \delta \Theta \mathrm{d} x^{a} \mathrm{~d} \rho+R^{2} \partial_{a} \delta \Theta \partial_{b} \delta \Theta \mathrm{d} x^{a} \mathrm{~d} x^{b} .
\end{aligned}
$$

This coincides with the result found in [14] at zero density.

To obtain a consistent solution at non-zero density, it is necessary to also include fluctuations in the gauge field $\delta A_{0}$ since these couple to the fluctuations $\delta \Theta$ of the induced metric of the D7-brane [24]. The coupling occurs since both fields transform as scalars under the group of rotations $\mathrm{SO}(3)$. They cannot be distinguished by the different transformation under the $\mathrm{U}(1)$ gauge symmetry anymore since this symmetry is broken at finite density. We may also think of the embedding scalar as being effectively charged and this explains its coupling to the gauge field fluctuations. Then the action in this case differs from the action in [14] by the non-vanishing gauge field and gauge field fluctuation terms. The action can be found in appendix D. The equation of motion is given by

$$
0=\partial_{\rho}\left[A \partial_{\rho}(\delta \Theta)\right]+B \omega^{2}(\delta \Theta)+C(\delta \Theta)
$$

where

$$
\begin{aligned}
& A=\frac{\rho^{5} f \tilde{f}\left(1-\chi^{2}\right)^{3}}{\left(1-\chi^{2}+\rho^{2} \chi^{\prime 2}\right)^{3 / 2} \sqrt{1-\frac{8 \tilde{d}^{2}}{\rho^{6} \tilde{f}^{3}\left(1-\chi^{2}\right)^{3}+8 \tilde{d}^{2}}}} \\
& B=\frac{\rho \tilde{f}^{2}\left(1-\chi^{2}\right)^{2}}{\sqrt{1-\chi^{2}+\rho^{2} \chi^{\prime 2} \sqrt{1-\frac{8 \tilde{d}^{2}}{\rho^{6} \tilde{f}^{3}\left(1-\chi^{2}\right)^{3}+8 \tilde{d}^{2}}}}}, \\
& C=\frac{3 \rho^{3} f \tilde{f}\left(1-\chi^{2}\right) \sqrt{1-\frac{8 \tilde{d}^{2}}{\rho^{6} \tilde{f}^{3}\left(1-\chi^{2}\right)^{3}+8 \tilde{d}^{2}}}}{\sqrt{1-\chi^{2}+\rho^{2} \chi^{\prime 2}}}\left[1-6 \chi\left(\rho \frac{f}{\tilde{f}} \chi^{\prime}+\chi\right)\right],
\end{aligned}
$$

with $f$ and $\tilde{f}$ defined in equation (3.2). For a more detailed derivation of the Lagrangian we refer to appendix D.

Now we can, similar to the procedure with the transversal vector modes, split the solution in a regulating and a regular part, and compute the asymptotic solution for the latter. This allows us then to apply the shooting technique (see A) to compute the quasinormal modes in the same manner as above.

Results for scalars. First we study the density dependence of the mode with purely imaginary quasinormal frequencies found at zero density in figure 7. Our numerical results are shown in figure 20. In this figure we observe that the critical parameter $\chi_{0}^{\text {crit }}$, at which the instability occurs, i.e. the quasinormal frequency enters the upper half plane, increases with the density. Thus we write $\chi_{0}^{\text {crit }}(\tilde{d})$. Also we note that at finite densities the modes become stable again at some larger value for $\chi_{0}$, which we denote by $\chi_{0,2}^{\text {crit }}(\tilde{d})$. If we increase 


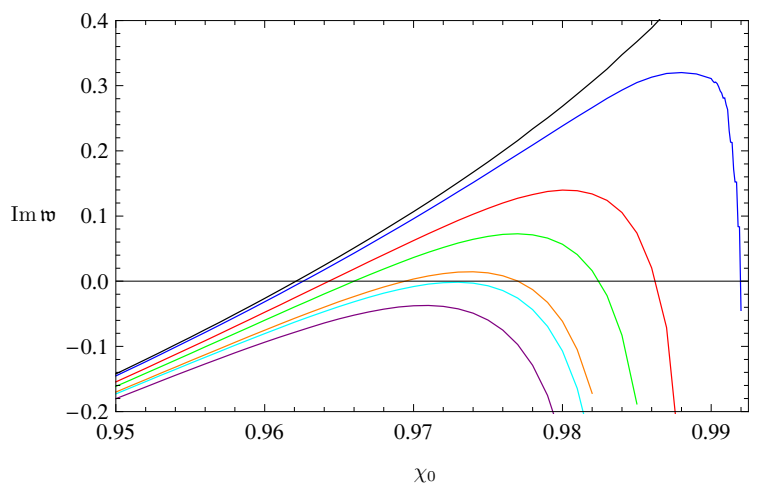

Figure 20. The scalar quasinormal modes with pure imaginary quasinormal frequencies for different densities $\tilde{d}$. For densities smaller than the critical density $\tilde{d}_{c}=0.00315$, the mode becomes tachyonic in some region of the parameter space. The several colors corresponds to different densities $\tilde{d}=0$ (black), 0.001 (blue), 0.002 (red), 0.0025 green, 0.003 (orange), 0.00315 (cyan), 0.0035 (purple).

the density further, we obtain a critical density $\tilde{d}_{c}=0.00315$ at which the mode is always stable. Therefore the system is unstable in the parameter region $\chi_{0}^{\text {crit }}(\tilde{d})<\chi_{0}<\chi_{0,2}^{\text {crit }}(\tilde{d})$ and $\tilde{d}<\tilde{d}_{c}$. The numerical values can be obtained from figure 20 . In section 6.5 as well as in the introduction, we further discuss this mode and relate the instability found to an instability already known in thermodynamics.

Let us now consider the behavior of the first quasinormal mode if we vary the density $\tilde{d}$. In figure 21 we present our numerical results. In this figure we observe that for each finite density we find that for large enough $\chi_{0}$ the quasinormal frequencies behaves in a similar way. The real part increases while the imaginary part decreases as we increase $\chi_{0}$. For smaller values of $\chi_{0}$ we observe three distinct movements of the quasinormal frequencies. For small densities $\tilde{d}<0.1$ the quasinormal frequency first follows the line of the quasinormal mode corresponding to zero density as we increase the parameter $\chi_{0}$. After a critical value of $\chi_{0}$ is reached the quasinormal frequency leaves this line as the real part of the quasinormal frequency increases monotonically. For slightly larger densities $0.11<\tilde{d}<0.2$, the frequencies also first moves along the line of the quasinormal mode at zero densities but in contrast to the case discussed above the real part of the frequencies now first decreases. For even higher densities $\tilde{d}>0.2$, the frequency at $\chi_{0}=0$ strongly depends on the densities. We find from figure 21 (c) that both the real and imaginary part of the quasinormal frequencies at $\chi_{0}=0$ increase with $\tilde{d}$. As we increase $\chi_{0}$ we find the usual behavior, the real part of the frequency increases while the imaginary part decreases.

\subsection{Schrödinger potential analysis}

In this section we present the Schrödinger potential analysis which we introduced in section 4 at finite baryon density. We use this analysis to explain the qualitative movement of the quasinormal frequencies as we change the baryon density.

For the vector fluctuations we observe in figure 19 (a) a turning point in the movement 


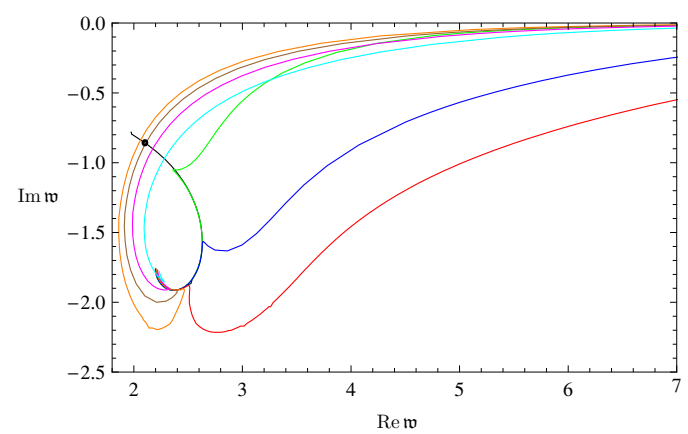

(a)

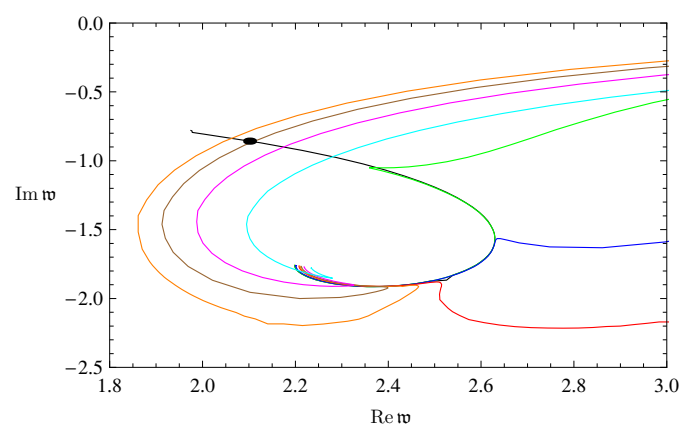

(b)

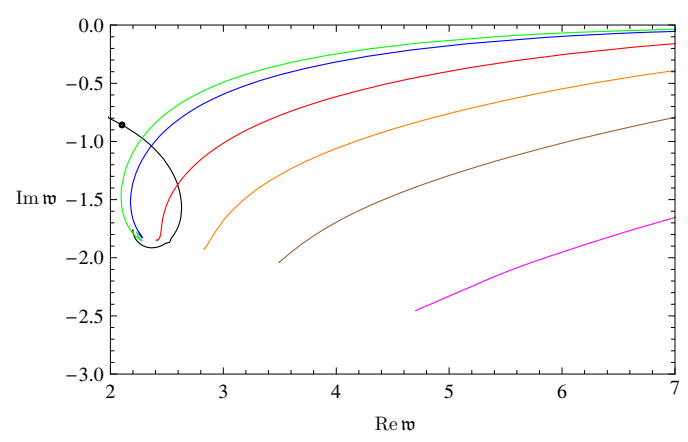

(c)

Figure 21. Dependence of the first scalar quasinormal frequency on the density: in (a) and (b) the quasinormal frequencies for small densities $\tilde{d} \leq 0.2$ are shown while in (c) densities up to $\tilde{d}=5$ are considered. In (a) and (b) the different colors corresponds to distinct densities $\tilde{d}=0$ (black), 0.01 (green), 0.05 (blue), 0.1 (red), 0.11 (orange), 0.125 (brown), 0.15 (magenta), 0.2 (cyan). In (c) the color coding is $\tilde{d}=0$ (black), 0.2 (green), 0.25 (blue), 0.5 (red), 1 (orange), 2 (brown), 5 (magenta). In all three plots the black dot marks the critical value of of the quark mass/temperature parameter $\chi_{0}$ where the instability occurs.

of the quasinormal frequencies at low baryon density. The real part of the quasinormal frequencies first increases and later decreases as we increase the mass parameter $m$. This turning point disappears as the critical density of $\tilde{d}_{c}=0.04$ is reached. After a critical quark mass is reached the real part of the quasinormal frequencies always increases while the imaginary part decreases.

Let us now consider the Schrödinger potential which correspond to these quasinormal modes in figure 22. A similar analysis was also done in [23]. In addition to the infinite wall at $R *=0$ which corresponds to the AdS boundary an additional peak appears in the potential as we increase the quark mass parametrized by $\chi_{0}$. For small densities (see figure 22 (a)) this peak slowly grows out of the step-shape potential already observed in section 4. The step-shape potential is also present at zero density and it is known from the analysis we presented there that the corresponding quasinormal frequencies show the turning point behavior discussed above. Thus the new feature of the finite density setup is the appearance of the peak at high quark masses. As the peak grows, 'bound' states with 


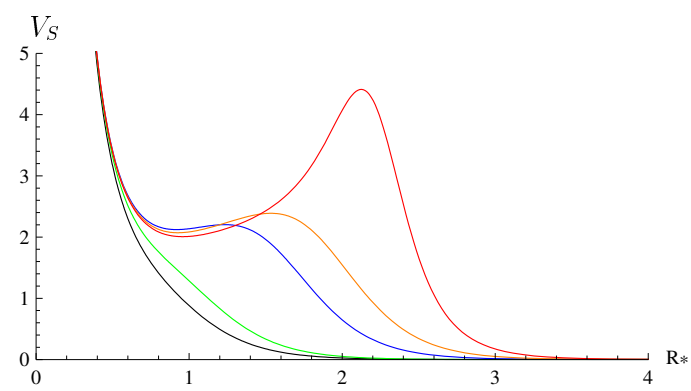

(a)

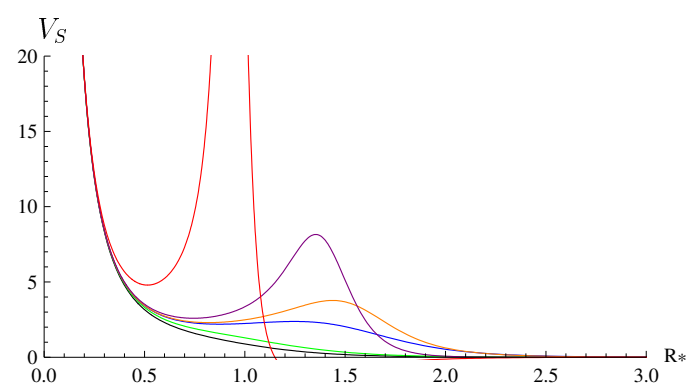

(c)

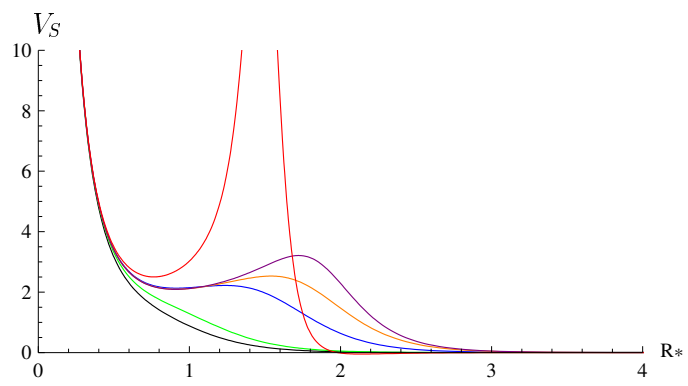

(b)

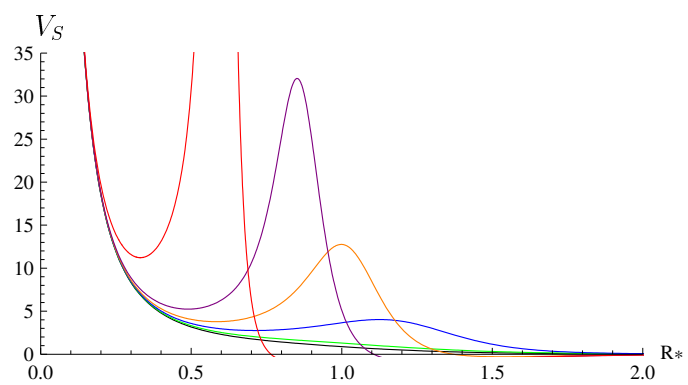

(d)

Figure 22. Schrödinger potential of the vector fluctuations at finite baryon density (a) $\tilde{d}=0.003$, (b) $\tilde{d}=0.01$, (c) $\tilde{d}=0.03$ and (d) $\tilde{d}=0.1$. The different colors corresponds to distinct quark masses parametrized by $\chi_{0}=0$ (black), 0.5 (green), 0.9 (blue), 0.95 (orange), 0.97 (purple), 0.99 (red). See figure 3 for the relation between $\chi_{0}$, the temperature and the quark mass $M_{q}$.

positive energy can be formed, i.e. the real part of the corresponding quasinormal frequency is always bigger than its imaginary part. Therefore we find quasiparticle excitations whose masses increase as we increase the quark mass.

If we increase the baryon density, the peak already appears at lower quark mass and can thus destroy the step-shape potential (see figure 22 (b) and (c)). For instance a step is still observable in the orange and blue curve in figure 22 (b) while in figure 22 (c) this step is gone. Since we know that the step-shape potential is the reason for the turning point potential, we also find in this analysis a critical baryon density at which the turning point disappears. This critical density agrees with the value found in figure 19 (a). For even larger densities (see figure $22(\mathrm{~d})$ ) the peak increases even faster, i.e. the real (imaginary) part of the corresponding quasinormal frequencies increase (decrease) even faster.

Let us now consider the scalar fluctuations at finite density which show several distinct features: a tachyonic mode at small densities and three qualitative different movements of the quasinormal frequencies.

From figure 20 we know that there exists a parameter region where a scalar mode becomes tachyonic. Especially interesting is that at finite density the mode is stabilized as the quark mass is increased and that there is a critical density $\tilde{d}=0.00315$ at which the mode is always stable. In figure 23 we present the Schrödinger potentials of the scalar fluctuations in the relevant density region. As in the case of zero density a negative well 


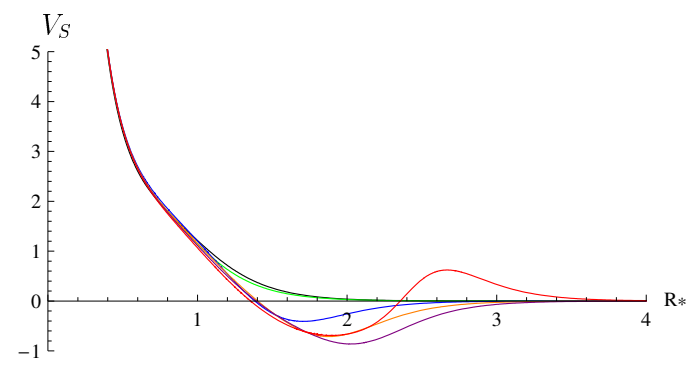

(a)

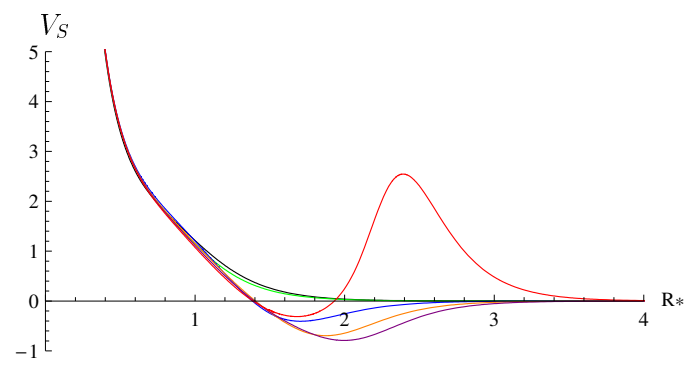

(b)

Figure 23. Schrödinger potential of the scalar fluctuations at finite baryon density (a) $\tilde{d}=0.002$, (b) $\tilde{d}=0.00315$. The different colors corresponds to distinct quark masses parametrized by $\chi_{0}=0$ (black), 0.5 (green), 0.9 (blue), 0.95 (orange), 0.97 (purple), 0.99 (red). See figure 3 for the relation between $\chi_{0}$, the temperature and the quark mass $M_{q}$.

appears in the potential. It also first grows as we increase the quark mass (see figure 23 (a)). However as we increase the quark mass further, the well decreases while a peak forms in the potential. After a critical quark mass is reached, this well cannot longer support a 'bound' state with negative energy, i.e. the imaginary part of the quasinormal frequency becomes negative again. At the critical density $\tilde{d}=0.00315$ (see figure 23 ), the potential also shows a negative well. However this well just reaches a critical depth in order to support a zero energy 'bound' state which is due to the zero-point energy (cf. the three dimensional potential pot know from quantum mechanics).

Now we investigate the Schrödinger potentials which are relevant for the movement of the first quasinormal frequency studied in figure 21. These potentials are plotted in figure 24. The first observation is that for small densities $\tilde{d} \lesssim 0.15$ (see figure 24 (a) and (b)) the potentials at low quark mass agree with the potential at zero density. Thus also the corresponding quasinormal frequencies must agree which we already found in figure 21 . At larger densities (see figure 24 (c)) even the potential at zero quark mass differs from the one at the zero density such that the corresponding quasinormal frequencies at zero quark mass depend on the density which is consistent with the result found in figure 21 (c). As for the vector fluctuations a peak appears in the potential at finite density as we increase the quark mass. These peak can again support 'bound' states which correspond to quasiparticle excitations. Thus the imaginary part of the quasinormal frequencies decreases while the real part increases as we increase the quark mass. This is the overall movement of the quasinormal frequencies which is shown in figure 21.

In general we observe that at finite baryon density a peak appears in the Schrödinger potential as we increase the quark mass. If the peak is high enough, it separates the horizon of the black hole from the AdS boundary. Only a small leak into the black hole is possible due to tunneling. The potential thus approaches the one of a Minkowski embedding where the brane does not fall into the horizon of the black hole and therefore can support stable normal modes which are calculated in [8] in the supersymmetric limit. In figure 25 we explicitly confirm that the Schrödinger potential obtained from the black hole embeddings 


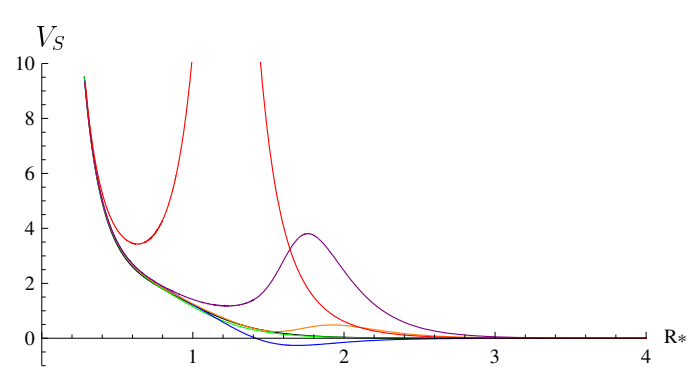

(a)

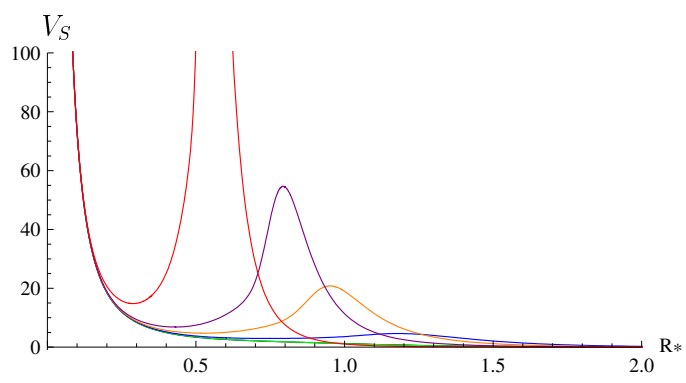

(b)

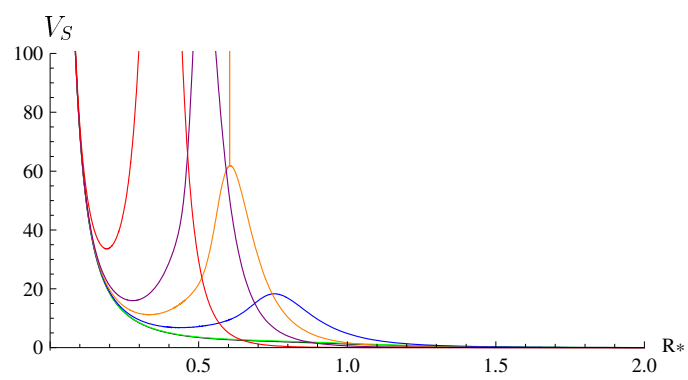

(c)

Figure 24. Schrödinger potential of the scalar fluctuations at finite baryon density (a) $\tilde{d}=0.01$, (b) $\tilde{d}=0.15$, (c) $\tilde{d}=0.5$. The different colors corresponds to distinct quark masses parametrized by $\chi_{0}=0$ (black), 0.5 (green), 0.9 (blue), 0.95 (orange), 0.97 (purple), 0.99 (red). See figure 3 for the relation between $\chi_{0}$, the temperature and the quark mass $M_{q}$.

converges to the potential of the supersymmetric Minkowski embedding ${ }^{7}$ with the same mass parameter $m$ as the mass parameter goes to infinity. This convergence explains that also the quasinormal frequencies approach this supersymmetric mass spectrum if the quark mass is big enough. This behavior was already found for the vector fluctuations in $[23,30]$. We can also understand this convergence if we look at the phase diagram (see figure 26). For large quark mass over temperature ratios the equal density lines approach the shaded region where the Minkowski embeddings are preferred. Since the phase transition is third or second order for large quark mass over temperature ratios as it is shown in [21], we also expect a smooth transition from the spectrum of the quasinormal modes obtained from the black hole embeddings to the spectrum of the normal modes obtained from the Minkowski embeddings.

Next we would like to understand the appearance of the peak in terms of the D7-brane embedding. In [16] it was shown that the finite baryon density on the brane is induced by fundamental strings which are stretched from the horizon of the black hole to the D7brane. At large quark masses these strings form a throat close to the horizon. We confirm

${ }^{7}$ The Schrödinger potential of a supersymmetric Minkowski embedding is given by [25]

$$
V_{S}=m^{2}\left[1 / 4+3 / 8\left(\tan ^{2}(m R * / \sqrt{2})+\cot ^{2}(m R * / \sqrt{2})\right)\right] .
$$




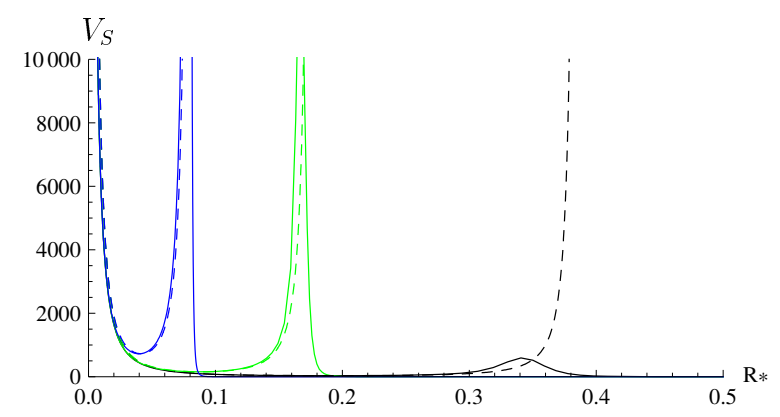

Figure 25. Comparison of the Schrödinger potential obtained from black hole embeddings (solid lines) and from supersymmetric Minkowski embeddings (dashed lines) at the same quark mass $m$. The different colors correspond to different quark masses parametrized by $\chi_{0}=0.99$ (black), 0.999 (green), 0.9999 (blue) at a fixed density $\tilde{d}=0.5$. See figure 3 for the relation between $\chi_{0}$, the temperature and the quark mass $M_{q}$.

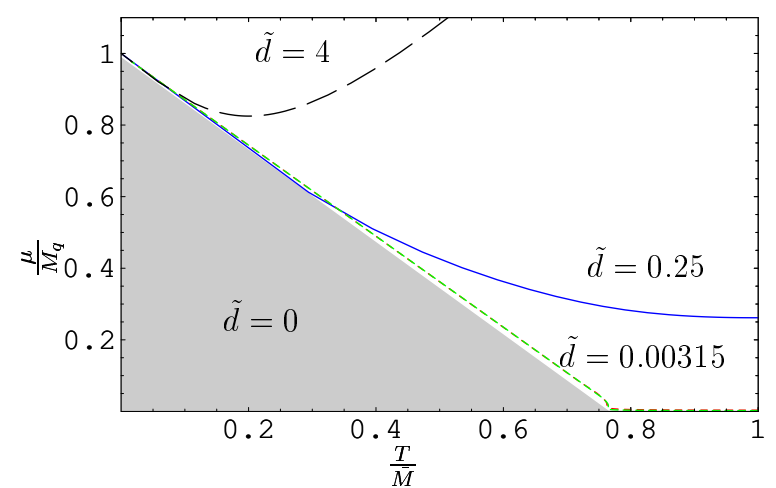

Figure 26. Sketch of the phase diagram: the chemical potential $\mu$ divided by the quark mass $M_{q}$ is plotted versus the temperature $T$ divided by $\bar{M}=2 M_{q} / \sqrt{\lambda}$. Two different regions are displayed: the shaded region with vanishing baryon density where Minkowski embeddings are preferred and the region above the transition line with finite baryon density where the black hole embeddings are preferred. Here we work in the second phase. The curves are lines of equal baryon density parametrized by $\tilde{d}$.

numerically that the end of this throat and the peak in the Schrödinger potential are located at the same value of the radial coordinate $\rho$ (see figure 27). Thus we can interpret this throat as a potential barrier for the fluctuations which becomes bigger as the throat becomes deeper.

\subsection{Analytic solution at high frequencies}

Motivated by the numerical solution to the fluctuation equations of motion shown in figure 28, we suspect that this damped oscillating curve near the horizon can be approximated by a damped quasi-harmonic oscillator, i.e. we should be able to find an approximate equation of motion which is a generalization of the damped harmonic oscillator equation. By quasi-harmonic we mean that the oscillator is damped with the damping depending on the location of the mode in radial direction. From the observations in figure 29 we have already concluded that the amplitude changes rapidly near the horizon and change less in 


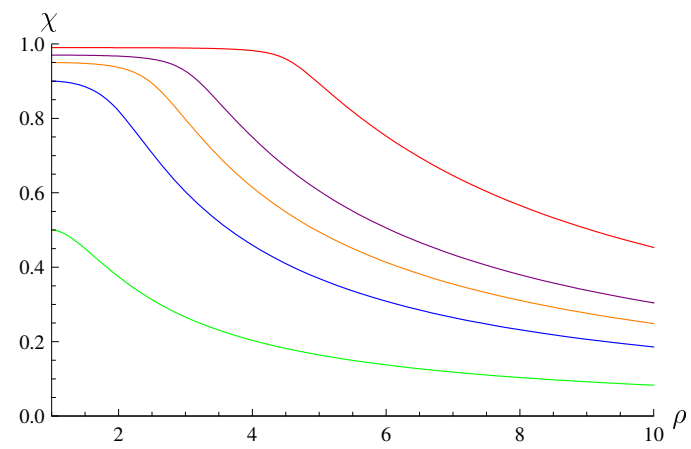

(a)

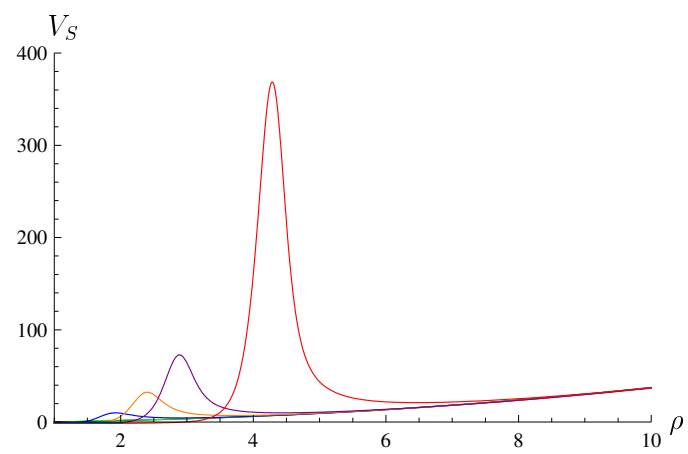

(b)

Figure 27. Comparison of the location of the peak in the Schrödinger potential and the throat in the embedding of the D7-brane. The end of the throat is located where $\chi$ first changes its value. The different colors corresponds to different quark masses parametrized by $\chi_{0}=0$ (black), 0.5 (green), 0.9 (blue), 0.95 (orange), 0.97 (purple), 0.99 (red). See figure 3 for the relation between $\chi_{0}$, the temperature and the quark mass $M_{q}$. The baryon density is $\tilde{d}=0.25$.
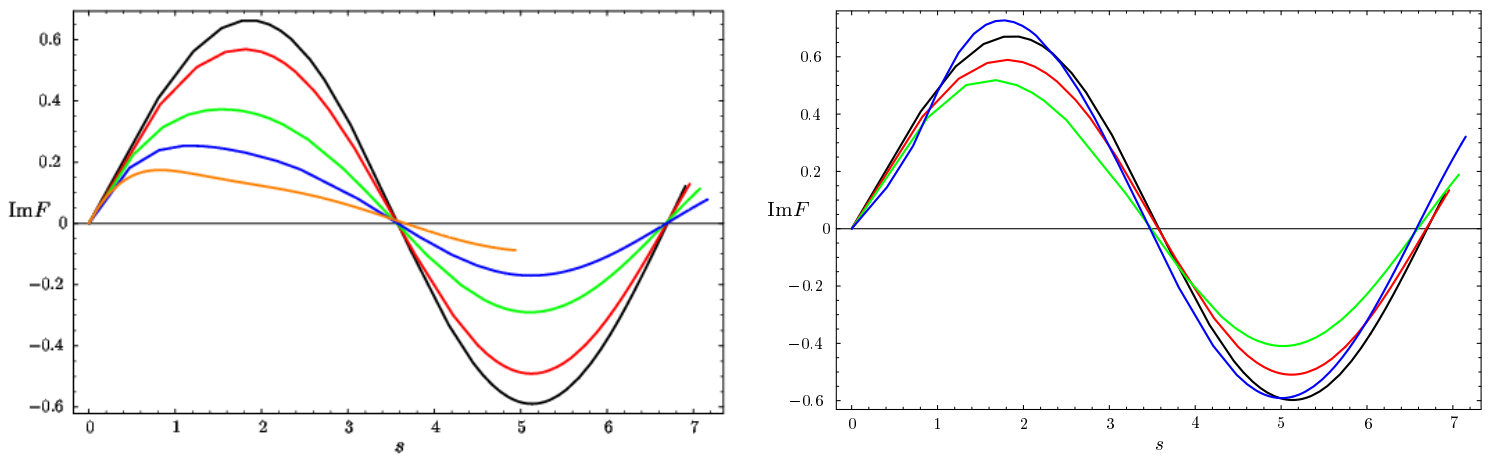

Figure 28. Imaginary part of the solution to the regular function $F$ versus the proper radial coordinate $s[38]$. The left plot at vanishing baryon density $\tilde{d}=0$ shows that the initially sinusoidal solution is deformed as the mass parameter $\chi_{0}=0.01,0.5,0.8,0.9$ is increased (see figure 3 for the relation between $\chi_{0}$, the temperature and the quark mass $M_{q}$ ). Furthermore, its amplitude decreases while the wave length increases. The right plot shows that introducing a finite baryon density $\tilde{d}=0.2$ causes the solutions to change their behavior with increasing $\chi_{0}$ : While the first three curves for $\chi_{0}=0.01,0.5,0.8$ show the same qualitative behavior as those in the left plot, the blue curve for $\chi_{0}=0.9$ clearly signals a qualitative change with its increased amplitude. Looking at the wave lengths in the lower plot we realize that already the green curve $\left(\chi_{0}=0.8\right)$ shows a decreased wave length as well as the blue curve $\left(\chi_{0}=0.9\right)$. The awkward oscillation pattern near $s=0$ stems from a second mode being superimposed on the one we are tracking here.

the bulk until the boundary is reached. Thus it is reasonable to assume that the damping of the mode $F$ already is strong near the horizon and a near-horizon approximation can capture this effect to certain extent. In this spirit we take the near-horizon limit $\varrho \sim 1$ and at the same time the high-frequency limit $\omega \gg 1$.

Applying these limits for the flat embedding $\chi_{0}=0$ in the equation of motion (6.5), 

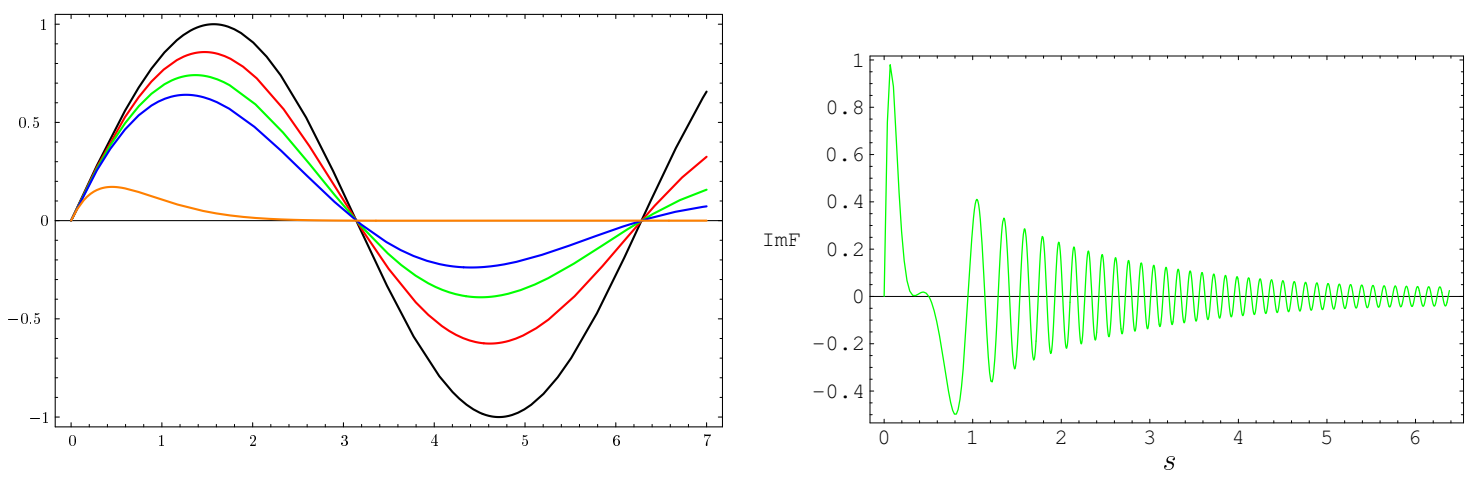

Figure 29. Left: solutions to the damped harmonic oscillator equation (6.14) for increasing damping coefficient $\gamma=0,0.1,0.2,0.3,0.9$ (black, red, green, blue, orange). Here the eigenfrequency $\omega$ is fixed to 1 in analogy to the plots in figure 28. Right: hi-frequency $(\omega=50)$ numerical solution showing that the damping of the mode is strong near the horizon $s=0$ but decreases exponentially in the bulk towards the boundary $s \rightarrow \infty$.

we obtain the simplified equation of motion

$$
y H^{\prime \prime}+(-i \omega-y) H^{\prime}+i \frac{\omega}{2}\left(\frac{1}{\sqrt{7}}+1\right) H=0
$$

where the variable is $y=i \omega \frac{\sqrt{7}}{4}(\varrho-1)$ and the regular function $H(y)$ comes from the Ansatz $E=(\varrho-1)^{\beta} F$ with the redefinition $F=e^{-\sqrt{7} / 4 i \omega(\varrho-1)} H$. This equation of motion has the form of Kummers equation, which is solved by the confluent hypergeometric function of first $H={ }_{1} F_{1}[-i \omega(1 / \sqrt{7}+1) / 2,-i \omega, \varrho-1]$ and second kind $U$. Boundary conditions rule out the second kind solution which is non-regular at the horizon and therefore contradicts the assumptions put into the Ansatz $E=(\varrho-1)^{\beta} F$. Since we are interested in how the solution changes with decreasing $m$, we need to choose $\chi_{0}$ non-vanishing. Also with this complication we still get Kummers equation with changed parameters and the analytic solution for $F$ is given by

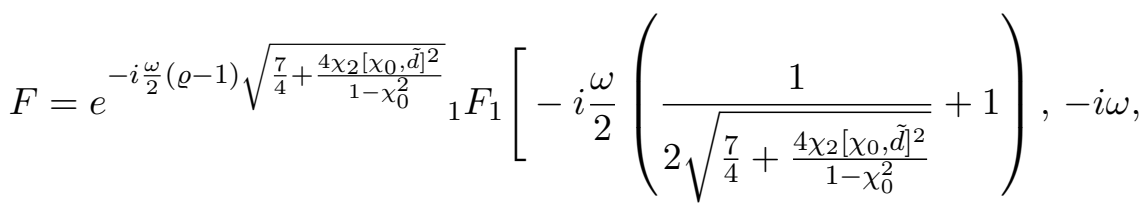

$$
\begin{aligned}
& \left.i \omega(\varrho-1) \sqrt{\frac{7}{4}+\frac{4 \chi_{2}\left[\chi_{0}, \tilde{d}\right]^{2}}{1-\chi_{0}^{2}}}\right],
\end{aligned}
$$

with the near horizon expansion of the embedding function $\chi=\chi_{0}+\chi_{2}\left[\chi_{0}, \tilde{d}\right](\varrho-1)^{2}+\ldots$ where we determine recursively

$$
\chi_{2}\left[\chi_{0}, \tilde{d}\right]=3 \chi_{0} \frac{\chi_{0}^{6}-3 \chi_{0}^{4}+3 \chi_{0}^{2}-1}{4\left(1-3 \chi_{0}^{2}+3 \chi_{0}^{4}-\chi_{0}^{6}+\tilde{d}^{2}\right)} .
$$

The approximate solution for $F$ is shown in figure 30. Furthermore we can calculate the fraction $\partial_{4} E / E$ appearing in the spectral function near the horizon using this analytic 


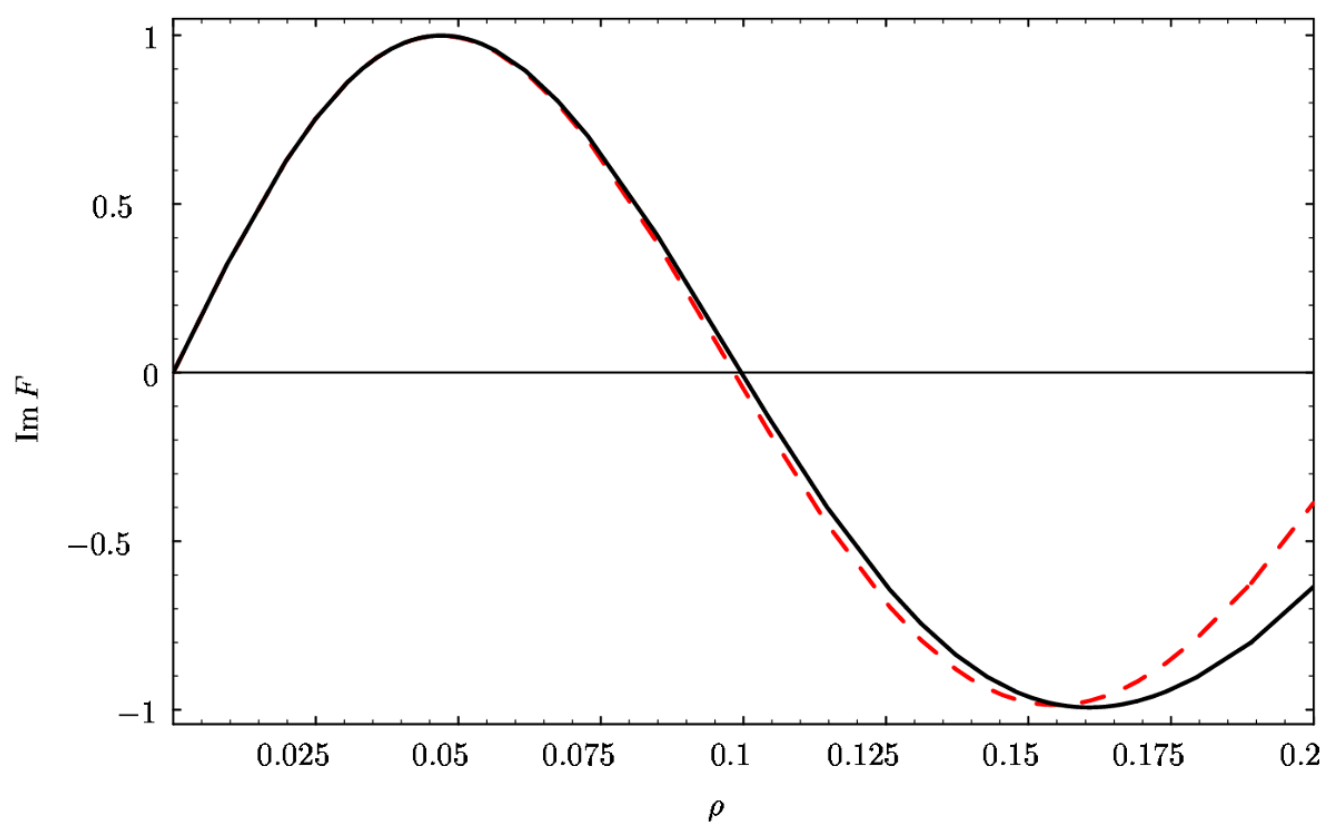

Figure 30. Approximate analytic solution compared to the exact solution at $\omega=70, \tilde{d}=0, \chi_{0}=$ 0.4 (see figure 3 for the relation between $\chi_{0}$, the temperature and the quark mass $M_{q}$ ).
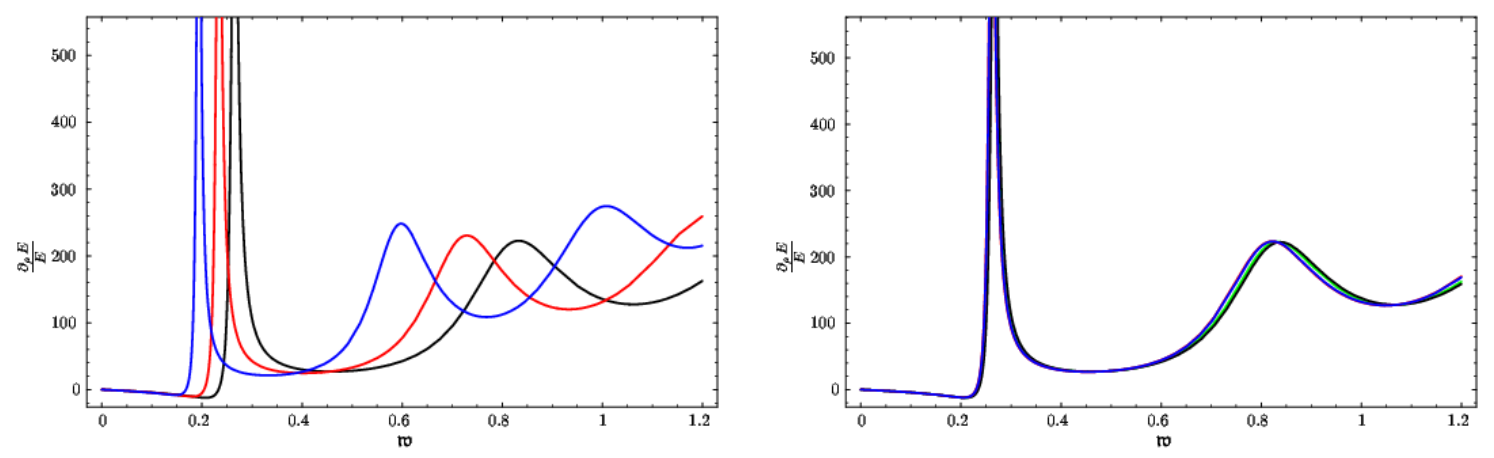

Figure 31. Approximate spectral function fraction near the horizon computed with the function $E=(\rho-1)^{\beta} F(\rho)$ and $F$ being the analytic approximation given in equation (6.11).

solution. The result is displayed in figure 31. This near horizon limit is not the spectral function since we would have to evaluate it at the boundary which lies far beyond the validity of the near horizon approximation. Nevertheless, in the spirit of [39] and according to our initial assumptions that the effect of damping mainly takes place near the horizon we further assume that the limit shown in figure 31 already contains the essential features of the spectral function. Indeed the fraction shows distinct resonance peaks which move to lower frequencies if we increase the mass parameter $m$. The right picture shows the same situation at a finite baryon density $\tilde{d}=1$ and we see that the peaks do not move to lower frequencies as much as before. Thus also the vanishing of the turning point at large densities as observed before is captured by this approximate solution. 


\subsection{Discussion: turning point and tachyon}

We now discuss the turning point in the spectrum of the vector modes and its dependence on the density. For scalars the purely imaginary scalar quasinormal mode reaches into the upper half of the complex plane and thus yields a tachyonic excitation, which we also discuss here.

\subsubsection{Turning point}

In this section we discuss why the vector mesons at finite temperature get smaller masses as the quark mass is increased. Further we investigate why there is a turning behavior at finite density. In the latter case we also find an analytic solution valid at high frequencies.

Line of argument. Let us first summarize our results. The D3/D7-system at finite density has two competing geometrical features. One is the formation of a potential barrier near the horizon due to the charge located at the horizon. This causes the resonances in the spectral function to become more stable and to move to larger frequency when the quark mass is increased. The second feature is the length of the brane ${ }^{8}$ supporting an excitation from the horizon to the boundary. Increasing this length causes the vector meson resonances to move to smaller frequency with increasing quark mass. These two geometrical features compete at finite density while at zero density the potential barrier is absent. Below we will explain this in greater detail and give a field theoretic interpretation.

Guiding features of numerical solutions. A detailed analysis of the numerical solutions [38] shows some interesting features. Most prominently there is a turning behavior, i.e. the location of the vector resonance peaks first move towards lower real frequencies, then turn around and move towards higher ones in order to asymptote to the SUSY-spectrum (increasing the quark mass to infinity is equivalent to the zero temperature case). This turning point for the lowest of the vector resonance peaks is only present at small densities $\tilde{d} \lesssim 1$. For larger densities the peaks run towards higher frequencies with increasing quark mass from the start, i.e. there is no turning point. As we see from the vector quasinormal modes the imaginary part monotonically decreases with increasing quark mass. At vanishing density the resonances only move to lower frequencies with increasing quark mass in the regime of black hole embeddings.

Therefore we have two things to explain: 1) the turning of the real part of the frequency and the decreasing imaginary part and 2) the motion towards smaller frequencies at zero density or small densities and quark masses $\left(\tilde{d}<1, \chi_{0}<0.5\right)$. The solutions $E$ of the bulk fluctuation equation of motion are composed of a singular part and the regular part $F$. From these solutions the spectral functions are computed. The regular part of the solutions has interesting properties, in particular:

\footnotetext{
${ }^{8}$ Since the AdS-boundary is infinitely far away from the horizon, the length of the brane has to be renormalized by subtracting for example the length of the brane at trivial embedding $\chi_{0}=0$.
} 


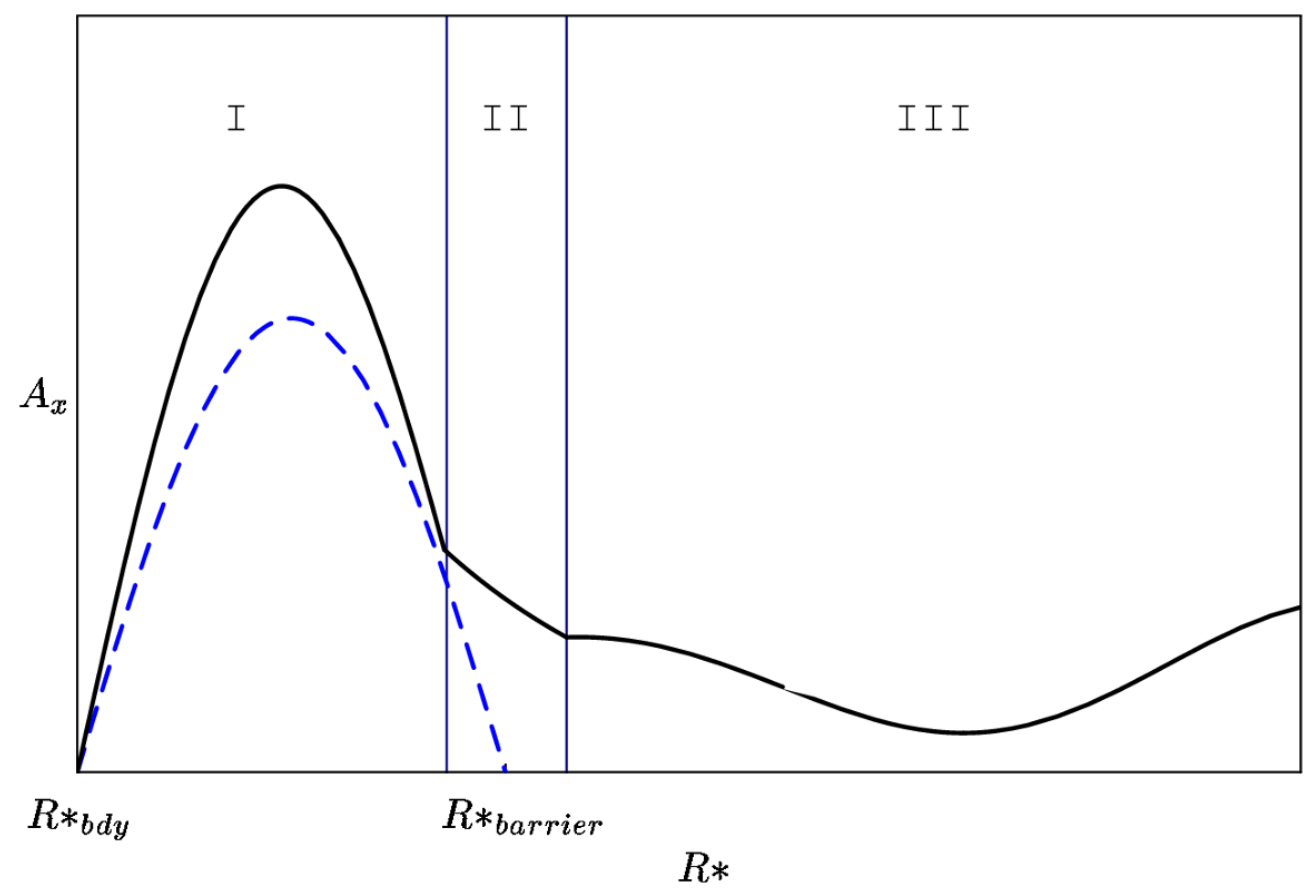

Figure 32. Schematic outline of the solution $A_{x}(R *)$ to the vector fluctuation equation of motion (6.5). The horizon is located at $R *=\infty$ where the wave function diverges, the AdS-boundary is $R *_{b d y}=0$. The solid black curve shows the solution at finite temperature, density and quark mass, i.e. on a black hole embedding. The dashed blue curve shows the corresponding solution on a Minkowski embedding at the same quark mass. The three regimes correspond to the nature of the potential shown in figure 25: I) Minkowski-like, II) potential barrier, III) essentially vanishing potential.

1. The proper distance $s$ on the brane

$$
s=\int_{\rho_{H}}^{\rho} \sqrt{G_{\rho \rho}} d \rho,
$$

increases with increasing quark mass parameter $\chi_{0}$. Integrating this expression from the horizon, to the boundary gives the proper length of the D7-brane. This also changes the wavelength of the fluctuations on the brane, as we will explain below.

2. As we see from figure 28 the form of the numerical solutions written in the proper radial coordinate $s$ shows a damped oscillation. Thus we are motivated to map the fluctuation equation of motion to a damped harmonic oscillator equation of motion.

3. At vanishing density $\tilde{d}=0$ a larger quark mass parameter $\chi_{0}$ induces a stronger damping of the solutions $F(s)$ (see figure 28, left).

4. At finite density a larger quark mass parameter $\chi_{0}$ first induces a stronger, then a weaker damping of the solutions $F(s)$ (see figure 28 , right). 
5. As seen from the high frequency $(\omega=50)$ solution in figure 29 most of the damping occurs close to the horizon while the fluctuation propagates towards the boundary with exponential damping.

6. At $\tilde{d}=0$ for $\chi \rightarrow 1$ black hole embeddings asymptote to $m=\left.\rho \chi\right|_{\rho \rightarrow \rho_{b d y}} \approx 1.3$; i.e. this geometric construction can not produce the $T \rightarrow 0$ limit in the black hole embeddings. Furthermore, long before $\chi_{0} \rightarrow 1$ one has to jump to Minkowski embeddings at the temperature where the instability appears. In other words: at fixed $T$ you can not make the quarks heavier than $M_{q} \sim 1.3 T$. And there is no smooth transition through the singularity at the limiting embedding $\chi_{0}=1$ to the Minkowski embeddings.

Resonances moving to higher frequencies towards the SUSY spectrum. At finite density there are two effects present which compete. One effect is the elongation of the brane. This is discussed below for the case of vanishing density. A specialty at finite density is the appearance of a longer and longer spike in the embedding reaching down to the horizon, connecting the Minkowski-like bulk part of the black hole embeddings with the horizon.

The second and as it turns out also the more important effect at finite density is the formation of a potential barrier near the horizon at $R *=\infty$, see figure 25 . This barrier effectively cuts off the horizon from the geometry. Only a small part of the wave function "leaks" into the region behind the potential barrier where the black hole is located, that is region $I I I$ in the schematic solution shown in figure 32. In this region III the Schrödinger potential asymptotically vanishes. Therefore the solution shown in figure 32 first drops to low values in order to diverge only very close to the horizon (not shown in the figure). Region $I I$ is the finite radial distance covered by the potential barrier. Here the wave function qualitatively drops exponentially. In region $I$ the main part of the solution is located between the boundary and the barrier. Here the potential approaches a pot-like form more and more similar to the corresponding potential generated by the Minkowski embedding as the quark mass parameter is increased. As this happens, the black hole fluctuation solution (solid black curve in the schematic plot in figure 32) approach the Minkowski solutions (dashed blue curve in the schematic plot in figure 32).

As we see from figure 27 the potential barrier moves closer to the boundary as the mass parameter $\chi_{0}$ is increased. Thus the potential gets more narrow and the lowest possible excitation is raised to a higher energy, i.e. the real part of the corresponding quasinormal mode is increasing. Furthermore the barrier becomes higher such that the corresponding excitation becomes more stable, i.e. the imaginary part of the quasinormal frequency decreases. In other words, less of the wave function leaks over the barrier into the black hole.

Resonances moving to lower frequencies. Let us for simplicity work at vanishing density first. All our considerations will also apply to the finite density case. The heuristic explanation for the left-movement of resonances has to do with the proper length of the D7-brane. At $\chi_{0}=0$ we have a flat embedding which is the minimal length the brane 
can have when measured in $s$. Increasing the quark mass parameter $\chi_{0}$ the D7-brane becomes "longer" in the sense that the proper length computed in the coordinate $s$ increases. Therefore we can imagine a solution supported on the brane to be "stretched" together with the brane, i.e. its effective wavelength increases. Assuming a constant effective speed of light, the effective frequency of this solution has to decrease. In this sense $\chi_{0}$ here acts analogously to a damping coefficient $\gamma$ in a damped harmonic oscillator. ${ }^{9}$

We may quantify this intuition examining the solutions $F(s)$ shown in figure 28. Indeed, comparing to the solutions (figure 29) of the damped harmonic oscillator equation

$$
0=\partial_{t}^{2} X(t)+2 \omega_{0} \gamma \partial_{t} X(t)+\omega_{0}^{2} X(t)
$$

with damping coefficient $\gamma$, we find that increasing $\chi_{0}$ in figure 28 resembles the effect of increasing the damping coefficient $\gamma$ in the analogous harmonic oscillator solutions, figure 29.

This leads us to assume that the fluctuation equation of motion on the D7-brane for real values of the frequency $\omega \in \mathbf{R}$ can effectively be replaced by the equation of motion for a damped harmonic oscillator with an effective eigenfrequency $\omega_{\text {eff }}$ and with the replacements $\gamma \rightarrow \gamma\left(\chi_{0}\right)$, with $\gamma\left(\chi_{0}\right)$ a monotonous function and $\omega_{0} \rightarrow \omega\left(\chi_{0}=0\right)$. By $\omega\left(\chi_{0}=0\right)$ we mean the effective eigenfrequency corresponding to the solution mainly influenced by the lowest of the quasinormal modes. $\gamma\left(\chi_{0}\right)$ can be seen as the effective damping coefficient. ${ }^{10}$ So the effective solution at finite $\chi_{0}$ reads

$$
F(s) \propto e^{-\gamma\left(\chi_{0}\right) \omega_{0} s} e^{i s \omega_{\mathrm{eff}}\left(\chi_{0}\right)}
$$

with the reduced eigenfrequency

$$
\omega_{\text {eff }}\left(\chi_{0}\right)=\omega_{0} \sqrt{1-\gamma\left(\chi_{0}\right)^{2}} .
$$

Since $0 \leq \gamma\left(\chi_{0}\right) \leq 1$ and $\gamma\left(\chi_{0}\right)$ is monotonous, the frequency of the solution which has the eigenfrequency $\omega_{0}$ at $\chi_{0}$ is monotonously decreasing with increasing $\chi_{0}$. So the quark mass parameter $\chi_{0}$ effectively acts as a damping coefficient. We might even suspect that the embedding $\chi(\rho)$ acts as a local damping coefficient depending on the radial location. In this way the fact that the damping is strongest at the horizon and vanishes towards the boundary (see figure 29, right) is consistent with the fact that the embedding $\chi(\rho)$ assumes its largest value near the horizon and quickly asymptotes to zero towards the boundary.

Now let us (as a very crude approximation) choose $\omega_{0}$ to be the real part of the first vector quasinormal mode on the D7-brane at vanishing $\chi_{0}$. The quasinormal mode is already damped, i.e. it actually has no real eigenfrequency. But let us nevertheless follow our

\footnotetext{
${ }^{9}$ This heuristic picture neglects the fact that the damping of the gravity modes in general is a local effect, i.e. it depends on the radial AdS-coordinate (through radially-dependent geometry). Tentatively we will assume that we can average the damping effects over the radial coordinate and express them globally in an effective damping coefficient independent from the radial coordinate.

${ }^{10}$ This identification of $\gamma\left(\chi_{0}\right)$ is effective in the sense that the actual quantity appearing in the equation of motion (6.5) is $\chi(\rho)$ which highly depends on the radial location. So we understand the effective damping $\gamma\left(\chi_{0}\right)$ to be a constant which averages damping effects over the whole AdS-radius.
} 
recipe and replace the complicated fluctuation equation by the simple damped harmonic oscillator with an effective damping $\gamma\left(\chi_{0}\right)$ and effective reduced eigenfrequency $\omega\left(\chi_{0}\right)$ from equation (6.16). The decreasing eigenfrequency $\omega\left(\chi_{0}\right)$ with increasing $\chi_{0}$ effectively explains the left-motion of the resonances in the corresponding spectral functions computed from these solutions $\operatorname{Im} G^{R} \propto \partial_{s} F(s) /\left.F(s)\right|_{s \rightarrow s_{b d y}}$. Here we have assumed that the lowest resonance peak in the spectral function behaves in the same way (moving to lower frequencies) as the effective eigenfrequency $\omega_{\text {eff }}\left(\chi_{0}\right)$ of the solution $F(s)$ from which it is computed. This intuition we get from the fact that in the exact computation both of these frequencies are mainly determined by the behavior of the lowest quasinormal mode.

These considerations shall serve to give an intuition for the behavior of the resonances. To be more precise the resonances are actually influenced by all the quasinormal modes, i.e. by their location in the complex frequency plane and by their residues.

\subsubsection{Killing the tachyon}

The scalar tachyon appearing at zero density is stabilized by introducing baryon charge. From a critical density $\tilde{d}_{c}=0.00315$ on the scalar does not become tachyonic for any value of $\chi_{0}$. Thus the finite charge density $\tilde{d}$ cures the instability and stabilizes the black hole phase of the D3/D7 system. ${ }^{11}$ We have described the scalar quasinormal mode signatures in detail in section 6.2. The mechanisms explaining this effect are discussed with the help of Schrödinger potentials in section 6.3. The negative potential well supporting the tachyon is lifted with increasing charge density. As discussed before the appearance of the tachyon is at non-zero density connected to the black hole to black hole first order phase transition taking place at finite densities $0<\tilde{d} \leq 0.00315$ between two distinct black hole phases. In particular the tachyon appears on the unstable branch of the free energy diagram of the phase transition. This branch connects two metastable branches as shown in figure 1. At the critical density $\tilde{d}_{c}=0.00315$ both the tachyon and the black hole to black hole phase transition disappear. It is not clear what is the physical ground state below $\tilde{d}_{c}$. As was argued in [17] the true ground state might be a mixed phase for which the gravity description is not known so far.

\section{Conclusions and outlook}

Our extensive study of the quasinormal modes of the D3/D7-brane system has revealed some interesting relations between previously known and unknown phenomena. As a main result we have shown that the system is completely stable above the critical density $\tilde{d}_{c}=0.00315$. That means that the spectrum of scalar excitations does not contain any tachyonic mode. Also in this regime the spectrum of mesonic exictations in the field theory -corresponding directly to distinct quasinormal modes- is de-singularized at low temperature or large quark mass through the explicit breaking of a scaling symmetry near the limiting embedding. i.e. the different meson excitations behave in accordance with the mass formula derived for the supersymmetric case [8]. Furthermore for the regime below

\footnotetext{
${ }^{11}$ Note that there exist Minkowski embeddings with the same chemical potential and the same quark mass, but all states in the Minkowski phase have strictly vanishing density $\tilde{d}=0[16,17]$.
} 
the critical density, i.e. for $\tilde{d}<0.00315$ we have established the connection between the black hole to black hole phase transition at finite charge density on one hand and the tachyonic scalar on the other. Using the Schrödinger formulation of the problem we have explained the movement of scalar and vector quasinormal modes in the complex frequency plane in great detail. A universal feature of all Schrödinger potentials at finite charge density is that they develop a barrier near the black hole horizon which hides the horizon and the black hole from the boundary. In consequence the dissipation decreases with increasing density and the quasinormal modes asymptote to a normal mode behavior. This behavior has also been observed in [40] in a setup where a black hole in $A d S_{4}$ develops scalar hair, i.e. there is also a charge density distributed near the horizon.

At vanishing baryon density but finite momentum we found a critical wavelength at which the hydrodynamic approximation explicitly breaks down. Below that wavelength the system at late times is no longer governed by hydrodynamic but by (propagating) collisionless modes only. A few unresolved issues remain for zero baryon density. In that case we found a spiraling behavior of the first quasinormal mode's trajectory for both scalars and vectors. The number $n$ of loops appears to be directly related to what we coined "attractor" frequencies $\omega_{n}$ to which all QNM-trajectories asymptote if their momentum $k_{n}$ lies in a certain momentum regime $k_{n-1}<k_{n} \leq k_{n+1}$. A direct relation remains unrevealed. In the same case we found that a scalar becomes tachyonic at low enough temperature. This instability might give rise to a condensation process or more generally to a phase transition as suggested by the hydrodynamic behavior of the scalar mode where it just becomes tachyonic. It is interesting to ask what the new phase could be and if it exists at all.

The analysis presented here can straightforwardly be extended to the D3/D7 setup with finite isospin density or with spontaneously broken symmetry [41, 42]. In these setups there is a variety of modes to be studied, in the latter most prominently the fluctuations of the order parameter phase which correspond to Goldstone modes becoming new hydrodynamic modes in the phase with spontaneously broken symmetry.

\section{Acknowledgments}

We are grateful to J. Mas, F. Rust, J. Shock, J. Tarrío for discussions. The work of K.L. M.K and F.P.B has been supported by Plan Nacional de Alta Energías FPA-200605485, Comunidad de Madrid HEPHACOS P-ESP-00346, Proyecto Intramural de CSIC 200840I257. F.P.B has also been supported by the Programa de apoyo institucional para cursar estudios de doctorado de la Universidad Simon Bolivar.

The work of J.E. C.G. and P.K. was supported in part by The Cluster of Excellence for Fundamental Physics - Origin and Structure of the Universe. The work of M.K. was supported in part by the German Research Foundation Deutsche Forschungsgemeinschaft (DFG).

\section{A Shooting method}

We now discuss an improved shooting method for solving the equation of motion for the fluctuations at complex frequencies. The problem arises when using the standard method 
for solving the equations of motion by just numerically integrating the differential equation.

As an example for the failure of this naive method we look at figure 33, which shows the spectral function for the transverse vector modes for vanishing quark mass and vanishing density. Note here that we use the dimensionless frequency $\mathfrak{w}=\omega_{p h} /(2 \pi T)=\omega / 2$ and momentum $\mathfrak{k}=k_{p h} /(2 \pi T)=k / 2$. The line of poles found at $\operatorname{Im} \mathfrak{w}=-1$ is definitely not correct since in [43] the pole-structure of the very same configuration was analytically determined to be

$$
\mathfrak{w}=n(1-\mathrm{i}) \text { for } n \in \mathbb{N}_{0} .
$$

It turns out that the numerical errors hide the quasi-normal modes behind this „wall“ at $\operatorname{Im} \mathfrak{w}=-1$. This wall could be misinterpreted as a branch cut.

The problem is, that the equations of motion we investigated are not regular at the horizon where the initial conditions are imposed. One therefore splits the solution in a regular and regulating part: $E(\rho)=(\rho-1)^{-\mathrm{iw}} F(\rho)$ by computing its Frobenius expansion near the horizon. Nevertheless, we have to move the starting point for the numerical integration slightly away from the real horizon to, say $\rho=1.00001$ where we choose this value so close to 1 that we can expect only small deviations from the exact solution. However, for values of $\operatorname{Im} \mathfrak{w} \leq-1$ the boundary condition yields

$$
(\rho-1)^{-\mathrm{i} \mathfrak{w}}=\left(1 \times 10^{-5}\right)^{\operatorname{Im} \mathfrak{w}-\mathrm{iRe} \mathfrak{w}} .
$$

This is problematic when Im $\mathfrak{w}$ is smaller than -1 , the initial condition is becoming large, leading to round off errors. These change the value of the fluctuation at infinity dramatically and are responsible for the invalid results of the spectral function in the specific region.

The cure is to shift the starting point for the numerical integration away from the horizon, and thus dealing with not so small values in the basis of (A.2), resulting in better numerical initial conditions. Of course, the error from starting the integration further away from the horizon has to be compensated. This is simply done, by calculating the asymptotics of the gauge field fluctuations in the vector case, or the embedding deviations in the scalar case at the horizon to higher order.

When reimplementing the numerical integration with a starting value of e.g. $\rho=1.1$ and the new asymptotics, the numerical aberration is prevented successfully and one can gain insight farther into the complex $\omega$-plane.

A look at the results shows what can be achieved with this more sophisticated method. A comparison between the surface plot of the old method (figure 33) and the improved one (figure 34) reveals that the expected pole structure can now be seen clearly. In fact it can be checked that the position of the poles agrees with the analytical result (A.1) very well.

\section{B Relaxation method}

The determination of quasinormal frequencies can be understood as a particular case of a two-point boundary value problem. A common numerical method for solving such problems is the shooting method, where one solves the differential equations with varying boundary 


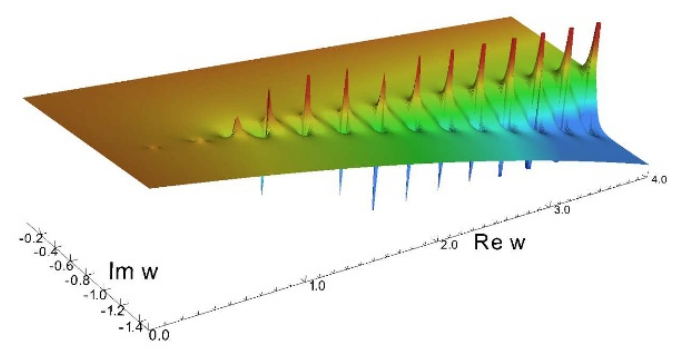

Figure 33. Breakdown of the standard numerical techniques at $\operatorname{Im} \mathfrak{w} \lesssim-1$. A series of spikes can be seen, being a firmly erroneous solution in view of the analytic result (A.1). This problem will be solved by means of our improved method, discussed in the text.

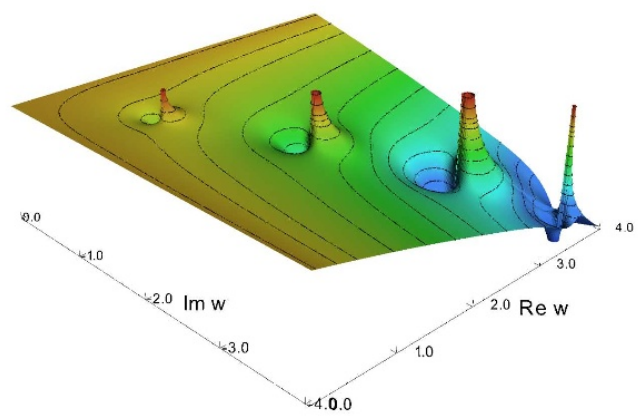

Figure 34. With the improved method nearly four poles can be resolved for vanishing density $\tilde{d}=0$ and massless quarks $m=0$. For the computation of the initial values the expansion in the fluctuation equation were evaluated up to eleventh order. As can be seen, the location of the poles fits the analytical solution (A.1) very well.

conditions at one boundary and searches for a solution that approximates the wanted boundary condition at the other boundary within some numerical error. We describe that method in appendix A.

A different approach is provided by the relaxation method which allows to fix the correct boundary values on both boundaries. Since this method is less frequently used we give a short outline below. ${ }^{12}$

The method is based on replacing the differential equations by a system of finitedifference equations (FDE) on a discrete grid. Starting from an ansatz solution obeying the correct boundary conditions, one varies the value of the dependent variables at each point relaxing to the configuration which provides an approximate solution for the FDE within some given numerical error. In our case, we convert our second order complex ODE into a set of four first order equations by separating real and imaginary parts of the dependent variables.

\footnotetext{
${ }^{12} \mathrm{~A}$ more detailed exposition can be found in [44].
} 
More generally we can consider a set of $\tilde{N}$ first order ODE's

$$
\frac{\mathrm{d} y_{i}(x)}{\mathrm{d} x}=g_{i}\left(x, y_{1}, \ldots, y_{\tilde{N}} ; \lambda\right)
$$

where each dependent variable $y_{i}(x)$ depends on the others and itself, on the independent variable $x$ and possibly on additional parameters, like $\lambda$ above. In our case this (complex) parameter is the quasinormal frequency. These extra parameters can be embedded into the problem by writing trivial differential equations for them

$$
\left\{\begin{aligned}
y_{N+j} & \equiv \lambda_{j}, \\
\frac{\mathrm{d} y_{N+j}}{\mathrm{~d} x} & =0, \text { since it is constant } .
\end{aligned}\right.
$$

We assume that there are $n$ additional parameters and include them from now on into the set of dependent variables $y_{1}, \ldots, y_{N}, N=\tilde{N}+n$.

The solution to the problem involves $N \times M$ values, for the $N$ dependent variables in a grid of $M$ points. We also have to fix $N$ boundary conditions for the dependent variables.

The system is discretized as usual

$$
x \rightarrow \frac{1}{2}\left(x_{k}+x_{k-1}\right), \quad y \rightarrow \frac{1}{2}\left(y_{k}+y_{k-1}\right),
$$

for points in the bulk. One may arrange the whole set of $y_{i}$ 's in a column vector $\mathbf{y}_{k}=$ $\left(y_{1}, \ldots, y_{N}\right)_{k}^{T}$, where the subscript $k$ refers to evaluation at the point $x_{k}, k=1, \ldots, M$. With this matrix notation, the system (B.1) can be written as

$$
0=\mathbf{E}_{k} \equiv \mathbf{y}_{k}-\mathbf{y}_{k-1}-\left(x_{k}-x_{k-1}\right) \mathbf{g}_{k}\left(x_{k}, x_{k-1}, \mathbf{y}_{k}, \mathbf{y}_{k-1}\right), \quad k=2, \ldots, M
$$

where the $\mathbf{E}_{k}$ are the aforementioned FDE's. These are the equations that we need to fulfil. Notice there are $N$ equations at $M-1$ points, so the remaining $N$ equations are supplied by the boundary conditions. We will set $n_{1}$ of them on the left at $x_{1}$, called $\mathbf{E}_{1}$, and the rest $n_{2}=N-n_{1}$ at $x_{M}$, called $\mathbf{E}_{M+1}$.

Now we take a trial solution that nearly solves the FDE's $\mathbf{E}_{k}$. By shifting each solution $\mathbf{y}_{k} \rightarrow \mathbf{y}_{k}+\Delta \mathbf{y}_{k}$ and Taylor expanding in the shift, one obtains the relation

$$
\begin{aligned}
0=\mathbf{E}_{k}(\mathbf{y}+\Delta \mathbf{y}) & \simeq \mathbf{E}_{k}\left(\mathbf{y}_{k}, \mathbf{y}_{k-1}\right)+\sum_{n=1}^{N} \frac{\partial \mathbf{E}_{k}}{\partial y_{n, k-1}} \Delta y_{n, k-1}+\sum_{n=1}^{N} \frac{\partial \mathbf{E}_{k}}{\partial y_{n, k}} \Delta y_{n, k}, \\
\Rightarrow-E_{j, k} & =\sum_{n=1}^{N}\left(S_{j, n} \Delta y_{n, k-1}\right)+\sum_{n=N+1}^{2 N}\left(S_{j, n} \Delta y_{n-N, k}\right)
\end{aligned}
$$

This allows to find the $\Delta \mathbf{y}_{k}$ that improve the solution. First we merge the two differentials

$$
S_{j, n}=\frac{\partial E_{j, k}}{\partial y_{n, k-1}}, \quad S_{j, n+N}=\frac{\partial E_{j, k}}{\partial y_{n, k}}, \quad n=1, \ldots, N
$$


in a $N \times 2 N$ matrix, for each bulk grid position $x_{k}$. For the boundaries the expressions follow equally

$$
\begin{aligned}
-E_{j, 1} & =\sum_{n=1}^{N} S_{j, n} \Delta y_{n, 1}=\sum_{n=1}^{N} \frac{\partial E_{j, 1}}{\partial y_{n, 1}} \Delta y_{n, 1}, \quad j=n_{2}+1, \ldots, N, \\
-E_{j, M+1} & =\sum_{n=1}^{N} S_{j, n} \Delta y_{n, M}=\sum_{n=1}^{N} \frac{\partial E_{j, M+1}}{\partial y_{n, M}} \Delta y_{n, M}, \quad j=1, \ldots, n_{2},
\end{aligned}
$$

where $n$ runs in both from 1 to $N$. The whole $(N M \times N M)$ matrix $S$ possesses a block diagonal structure. In fact since for reasonable systems $N<<M S$ is a sparse matrix. This allows the usage of computer packages in which the solution of linear systems with sparse matrices can be done in a very efficient way. The actual solution can now be found by an iterative process until a desired accuracy is achieved. As measure for the discrepancy of an approximate solution to the actual solution we used

$$
\operatorname{err}=\frac{1}{M N} \sum_{k=1}^{M} \sum_{j=1}^{N}\left|\frac{\Delta y[j][k]}{\text { scalevar }[j]}\right|<\text { conv }
$$

where scalevar $[j]$ is an associated scale for each of the dependent variables (e.g. the value at the midpoint or so). The idea is that when that averaged value of the shift to get a better solution is smaller than conv, we accept the former values we had as the actual solution. In our computations we set conv $=10^{-6}$.

In order to obtain the correct boundary conditions we have used the $z$ coordinate system where the horizon lies at $z=1$ and the boundary at $z=0$. We split off the ingoing boundary condition on the horizon according to $\Phi(z)=(1-z)^{-i \omega / 4} y(z)$. Demanding $y(1)=1$ and $y(0)=0$ gives four real boundary conditions. Since in total we have however six dependent variables, counting also the real and imaginary part of the quasinormal frequency we need two more boundary conditions. We found it convenient to expand the function $y(z)$ in a Taylor series at the horizon and compute also $y^{\prime}(1)$ which provides the additional two real boundary conditions. The typical gridsize we used was consisted of 5000 points. Finally we note that we have implemented to outlined algorithm in GNU/Octave [45].

\section{Schrödinger potentials}

This appendix shows how to compute the effective potentials, i.e. the Schrödinger potentials for the scalar and vector fluctuations on the D7-probe-brane. In order to do so the linearized fluctuation equations of motion have to be rewritten in terms of a new radial coordinate $R *$. This procedure has been described before (e.g. in $[14,25], \ldots$ ), nevertheless we include it here for completeness. For convenience we stick to the notation made use of in [14], and we compute all Schrödinger potentials in the $\rho$-coordinates introduced in section 3.

We are not interested in the higher angular excitations on the $S^{3}$, so we separate the fluctuations according to $\phi\left(\rho, S^{3}\right)=y(\rho) \Phi\left(S^{3}\right)$. Let us consider exclusively fluctuations 
without angular momentum on the $S^{3}$, i.e. $\Phi\left(S^{3}\right)=1$. All the linearized vector and scalar fluctuation equations of motion are second order ordinary differential equations and can be rewritten in the form

$$
-\frac{H_{0}}{H_{1}} \partial_{\rho}\left[H_{1} \partial_{\rho} y(\rho)\right]+\left[\mathfrak{k}^{2} H_{2}+H_{\theta}\right] y(\rho)=\mathfrak{w}^{2} y(\rho),
$$

where $H_{0}, H_{1}, H_{2}$ and $H_{\theta}$ are in general functions of $\rho$ and depend on the particular field fluctuation considered. $H_{\theta}$ only appears in the scalar fluctuations. For transverse vector fluctuations we have

$$
\begin{aligned}
& H_{0}=-\frac{g^{\rho \rho}}{g^{t t}}, H_{1}=\sqrt{-g} g^{\rho \rho} g^{x x}, \\
& H_{2}=-\frac{g^{z z}}{g^{t t}}, \quad H_{\theta}=0 .
\end{aligned}
$$

For longitudinal vector fluctuations we have

$$
\begin{aligned}
& H_{0}=-\frac{g^{\rho \rho}}{g^{t t}}, H_{1}=\sqrt{-g} g^{\rho \rho} g^{z z}, \\
& H_{2}=-\frac{g^{z z}}{g^{t t}}, \quad H_{\theta}=0 .
\end{aligned}
$$

For scalar fluctuations the equations of motion do not take such a simple general form in terms of metric components since the metric itself contains scalar fluctuations. So we choose to write explicitly

$$
\begin{aligned}
& H_{0}=-\frac{f^{2} \rho^{4}\left(1-\chi^{2}\right)}{8 \tilde{f}\left(1-\chi^{2}+\rho^{2} \chi^{\prime 2}\right)}, \quad H_{1}=\frac{f \tilde{f} \rho^{5}\left(1-\chi^{2}\right)^{3}}{\left(1-\chi^{2}+\rho^{2} \chi^{\prime 2}\right)^{3 / 2}} \sqrt{1+\frac{8 \tilde{d}^{2}}{\tilde{f}^{3} \rho^{6}\left(1-\chi^{2}\right)^{3}}}, \\
& H_{\theta}=\frac{3 f^{2} \tilde{f}^{2} \rho 8\left(1-\chi^{2}\right)^{2}\left[\tilde{f}^{3} \rho^{6}\left(1-\chi^{2}\right)^{3}+8 \tilde{d}^{2}\left(1-6 \chi^{2}\right)-144 \tilde{d}^{2} f^{3} \tilde{f} \rho^{9} \chi \chi^{\prime}\left(1-\chi^{2}\right)^{2}\right]}{8\left[\tilde{f}^{3} \rho^{6}\left(1-\chi^{2}\right)^{3}+8 \tilde{d}^{2}\right]^{2}}
\end{aligned}
$$

where we do not include $\mathrm{H}_{2}$ since the above coefficients are computed in the case of vanishing momentum $\mathfrak{k}=0$ but at finite density $\tilde{d}$. At finite momentum and at vanishing density we have $H_{2}=f^{2} / \tilde{f}^{2}$ in addition to the $\tilde{d} \rightarrow 0$ limits of $H_{0}, H_{1}$ and $H_{\theta}$ from (C.6). At finite momentum and density the scalar fluctuations couple to the vector fluctuations and we will not address this complication in this work.

In any case we can substitute $y(\rho)=h \psi$ in equation (C.1) to obtain

$$
-H_{0} \psi^{\prime \prime}-H_{0}\left(2 \frac{h^{\prime}}{h}+\frac{H_{1}{ }^{\prime}}{H_{1}}\right) \psi^{\prime}+\left[\mathfrak{k}^{2} H_{2}+H_{\theta}-H_{0}\left(\frac{h^{\prime \prime}}{h}+\frac{H_{1}{ }^{\prime}}{H_{1}} \frac{h^{\prime}}{h}\right)\right] \psi=\mathfrak{w}^{2} \psi .
$$

Introducing the new radial coordinate

$$
R *=\int_{\rho}^{\infty} d \tilde{\rho} / \sqrt{H_{0}(\tilde{\rho})},
$$

we can rewrite the first term $-H_{0} \psi^{\prime \prime}=-\partial_{R *}{ }^{2} \psi+H_{0}{ }^{\prime} \psi^{\prime} / 2$. The special choice of $h=$ $H_{0}{ }^{1 / 4} / H_{1}{ }^{1 / 2}$ eliminates all the terms containing $\psi^{\prime}$. Thus the fluctuation equation of motion finally assumes Schrödinger form

$$
-\partial_{R *}^{2} \psi+V_{S} \psi=E \psi
$$


with the effective energy $E=\mathfrak{w}^{2}$ and the Schrödinger potential given by

$$
V_{S}=-H_{0}\left(\frac{h^{\prime \prime}}{h}+\frac{H_{1}^{\prime}}{H_{1}} \frac{h^{\prime}}{h}\right)+\mathfrak{k}^{2} H_{2}+H_{\theta}
$$

From this general formula the specific scalar, longitudinal and transversal vector Schrödinger potentials may be obtained by substituting for $H_{0}, H_{1}, H_{2}, H_{\theta}, h$ using the corresponding values from equations (C.6), (C.4) and (C.2), respectively.

\section{Finite density but vanishing momentum}

To abbreviate the longish Lagrangian we introduced the following notation

$$
a=1-\chi^{2}, \quad b=a+\rho^{2} \chi^{\prime 2}, \quad c=\frac{8 \tilde{d}^{2}}{\rho^{6} \tilde{f}^{3} a^{3}+8 \tilde{d}^{2}}, \quad \text { so } \quad 1-c=\frac{\rho^{6} \tilde{f}^{3} a^{3}}{\rho^{6} \tilde{f}^{3} a^{3}+8 \tilde{d}^{2}} .
$$

In terms of these expressions the Lagrangian, expanded to second order, reads

$$
\begin{aligned}
\frac{\mathcal{L}}{\left(-N_{f} T_{D 7} \sqrt{h_{3}} \frac{r_{H}^{4}}{4}\right)}= & \mathcal{L}_{0}+\mathcal{L}_{1}+\mathcal{L}_{2}-\rho^{3} f \tilde{f} a \sqrt{b} \sqrt{1-c} F^{04}\left(\delta F_{40}\right)-\frac{3}{2} \rho^{3} f \tilde{f} \frac{a-b c}{\sqrt{b} \sqrt{1-c}}(\delta \theta)^{2} \\
& -\frac{R^{4}}{r_{H}^{2}} \rho \frac{\tilde{f}^{2}}{f} \frac{a^{2}}{\sqrt{b} \sqrt{1-c}}\left(\partial_{t} \delta \theta\right)^{2}+\frac{1}{2} \rho^{5} f \tilde{f} \frac{a^{2}(a-b c)}{b^{3 / 2}(1-c)^{3 / 2}}\left(\partial_{\rho} \delta \theta\right)^{2} \\
& +\frac{R^{2}}{2} \sum_{i} \mathcal{G}_{0}^{i i} \chi^{2}\left(\partial_{i} \delta \phi\right)^{2}+\rho^{5} \tilde{f}^{3 / 2} \chi^{\prime} \frac{\sqrt{2}}{r_{H}} \frac{a^{2} \sqrt{c}}{b(1-c)^{3 / 2}}\left(\delta F_{40}\right)\left(\partial_{\rho} \delta \theta\right) \\
& -\rho^{3} \tilde{f}^{3 / 2} \chi \frac{3 \sqrt{2}}{r_{H}} \frac{a \sqrt{c}}{\sqrt{1-c}}\left(\delta F_{40}\right)(\delta \theta)+\frac{1}{4} \rho^{3} f \tilde{f} a \sqrt{b} \sqrt{1-c} \sum_{i k} \mathcal{G}_{0}^{i i} \mathcal{G}_{0}^{k k}\left(\delta F_{i k}\right)^{2} \\
& -\rho^{3} f \tilde{f} a \sqrt{b} \sqrt{1-c} F^{04} \sum_{i} \mathcal{G}_{0}^{i i}\left(-\frac{\left(1+\delta_{4 i}\right) R^{2} \chi^{\prime}}{\sqrt{a}}\right)\left(\delta F_{i 0}\right)\left(\partial_{i} \delta \theta\right)
\end{aligned}
$$

where $\mathcal{L}_{0}$ is simply the Lagrangian without any fluctuations but nonvanishing density

$$
\mathcal{L}_{0}=\rho^{3} f \tilde{f} a \sqrt{b} \sqrt{1-c}
$$

and the boundary terms $\mathcal{L}_{1}$ and $\mathcal{L}_{2}$ are given by

$$
\mathcal{L}_{1}=\partial_{\rho}\left[-\frac{\rho^{5} f \tilde{f} a^{3 / 2} \chi^{\prime}}{\sqrt{b} \sqrt{1-c}}(\delta \theta)\right] \quad \text { and } \quad \mathcal{L}_{2}=\partial_{\rho}\left[-\frac{3}{2} \frac{\rho^{5} f \tilde{f} \chi \chi^{\prime} a}{\sqrt{b} \sqrt{1-c}}(\delta \theta)^{2}\right]
$$

The symbols denoted by $\mathcal{G}$ with upper indices are the components (diagonal part) of the inverse background tensor $\mathcal{G}=G+2 \pi \alpha^{\prime} F$. The most important ones are

$$
\mathcal{G}_{0}^{00}=-2 \frac{R^{2}}{r_{H}^{2}} \frac{\tilde{f}}{f^{2} \rho^{2}(1-c)} \quad \text { and } \quad \mathcal{G}_{0}^{44}=\frac{\rho^{2} a}{R^{2} b(1-c)} .
$$


For the off-diagonal part of the inverse background tensor $\mathcal{G}^{04} \equiv F^{04}=-F^{40}$ holds, because of the antisymmetry of the field strength tensor $F$, and has the value

$$
F^{04}=-\frac{\sqrt{2 \tilde{f} a c}}{r_{H} f \sqrt{b}(1-c)} .
$$

Finally, $\delta F_{i j}=\partial_{i} \delta A_{j}-\partial_{j} \delta A_{i}$ is the field strength for the vector fluctuation.

\section{E Results for second quasinormal modes}

In this appendix we collect results for the location of the second quasinormal modes (scalar, transverse and longitudinal vectors) at finite momentum but vanishing density.

\section{E.1 Transverse vector fluctuations}

Figure 36 shows the dispersion relation of the second transverse vector QNM at different values for the mass parameter $\theta_{0}$.
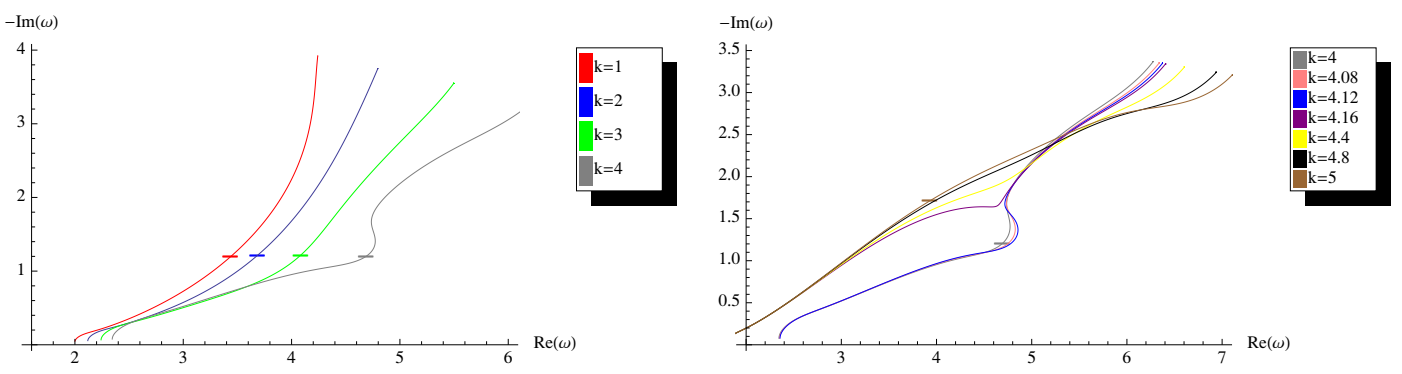

Figure 35. Second quasinormal mode of transverse vector fluctuations for distinct values of the spatial momentum $k$.
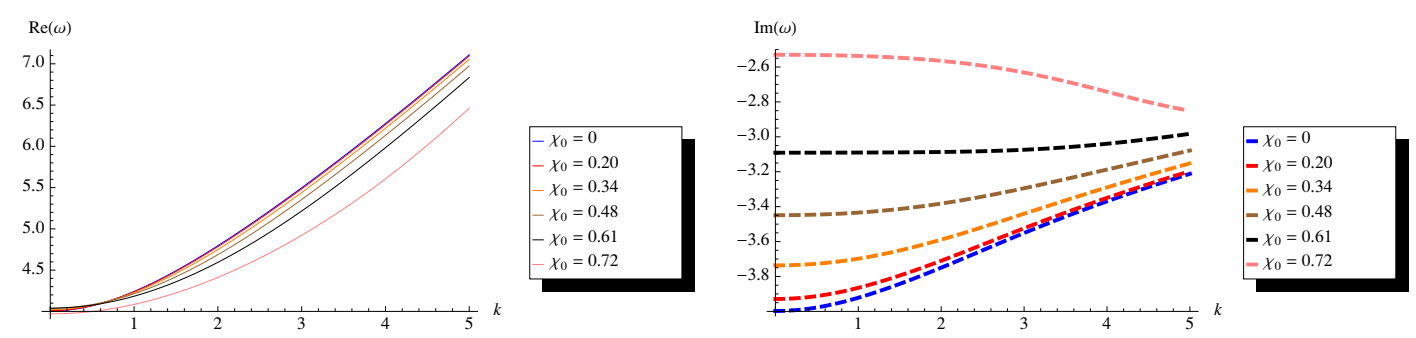

Figure 36. Dispersion relation for the second transverse vector quasinormal mode. 


\section{E.2 Longitudinal vector fluctuations}
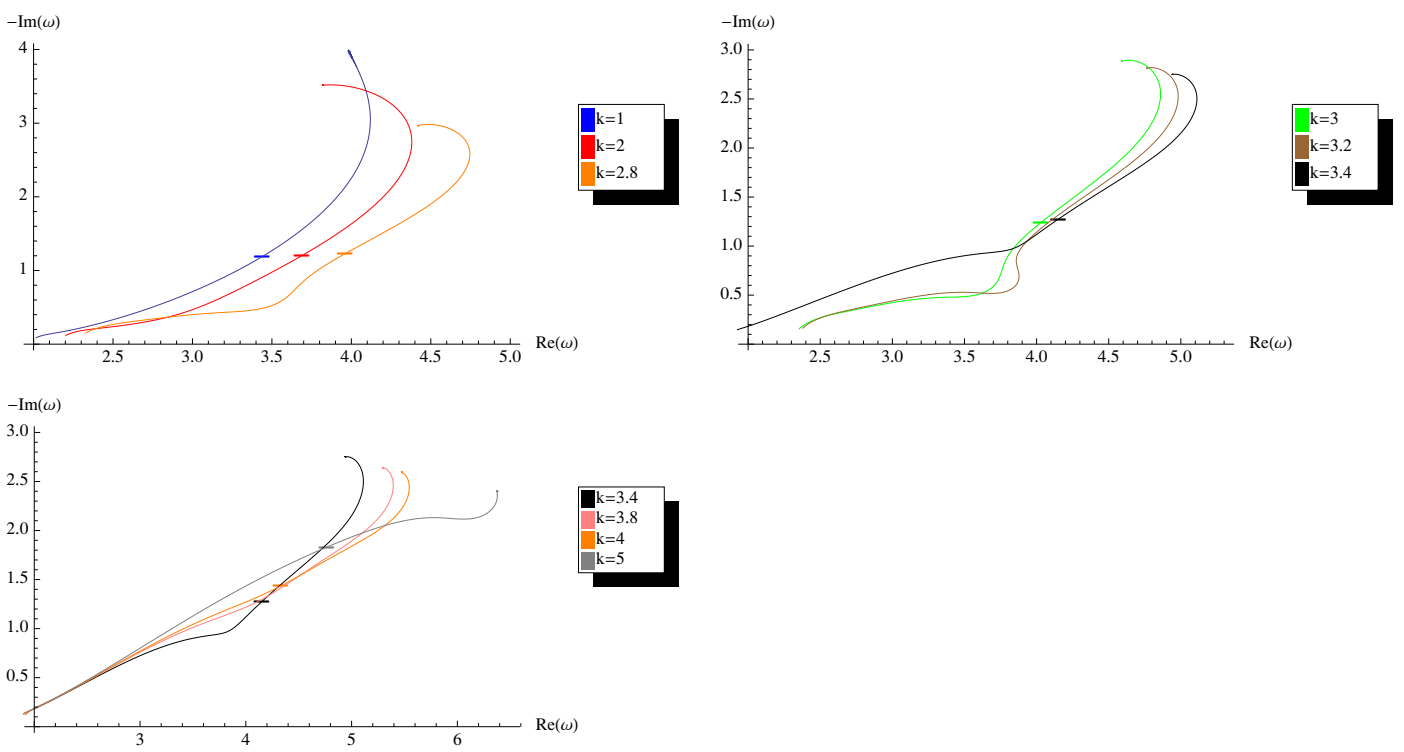

Figure 37. Second quasinormal mode for longitudinal vector fluctuations at distinct spatial momenta $k$.
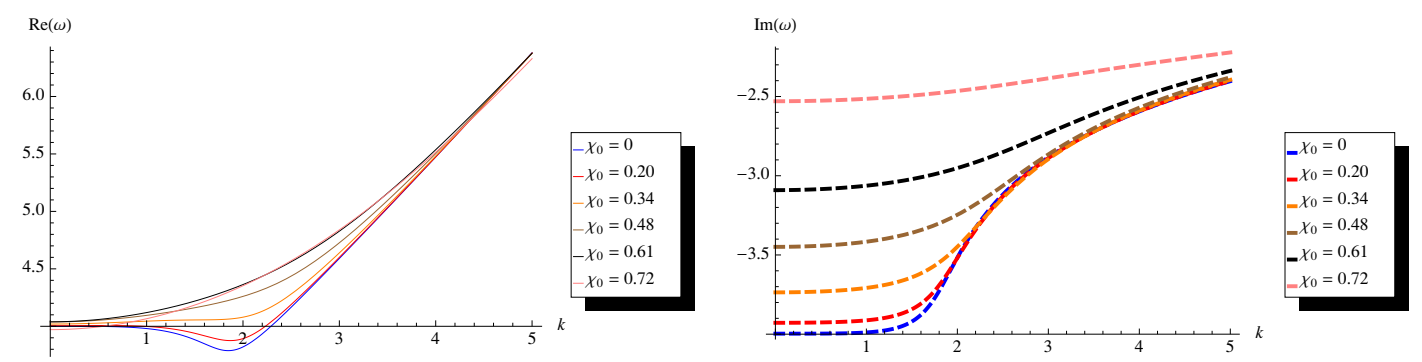

Figure 38. Dispersion relation for the second longitudinal vector quasinormal mode fluctuations at distinct spatial momenta $k$. 


\section{E.3 Scalar fluctuations}

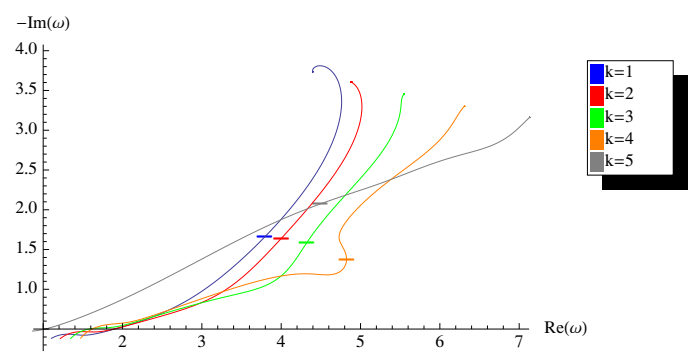

Figure 39. Second scalar quasinormal mode at distinct $k$.
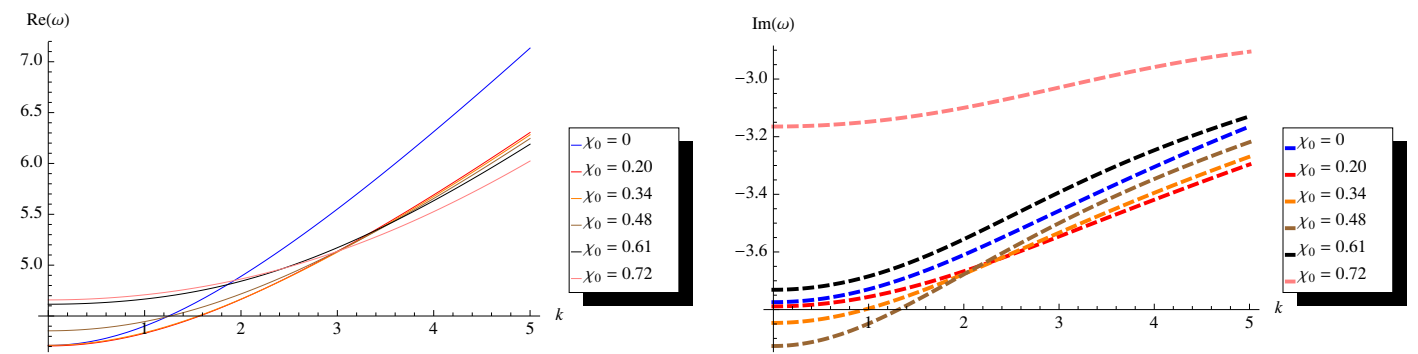

Figure 40. Dispersion relation for the second scalar quasinormal mode for distinct mass or temperature parameter $\theta_{0}$.

Open Access. This article is distributed under the terms of the Creative Commons Attribution Noncommercial License which permits any noncommercial use, distribution, and reproduction in any medium, provided the original author(s) and source are credited.

\section{References}

[1] J.M. Maldacena, The large- $N$ limit of superconformal field theories and supergravity, Adv. Theor. Math. Phys. 2 (1998) 231 [Int. J. Theor. Phys. 38 (1999) 1113] [hep-th/9711200] [SPIRES].

[2] O. Aharony, S.S. Gubser, J.M. Maldacena, H. Ooguri and Y. Oz, Large-N field theories, string theory and gravity, Phys. Rept. 323 (2000) 183 [hep-th/9905111] [SPIRES].

[3] G. Policastro, D.T. Son and A.O. Starinets, The shear viscosity of strongly coupled $N=4$ supersymmetric Yang-Mills plasma, Phys. Rev. Lett. 87 (2001) 081601 [hep-th/0104066] [SPIRES].

[4] E. Witten, Anti-de Sitter space, thermal phase transition and confinement in gauge theories, Adv. Theor. Math. Phys. 2 (1998) 505 [hep-th/9803131] [SPIRES].

[5] D. Birmingham, I. Sachs and S.N. Solodukhin, Conformal field theory interpretation of black hole quasi-normal modes, Phys. Rev. Lett. 88 (2002) 151301 [hep-th/0112055] [SPIRES]. 
[6] P.K. Kovtun and A.O. Starinets, Quasinormal modes and holography, Phys. Rev. D 72 (2005) 086009 [hep-th/0506184] [SPIRES].

[7] A. Karch and E. Katz, Adding flavor to AdS/CFT, JHEP 06 (2002) 043 [hep-th/0205236] [SPIRES].

[8] M. Kruczenski, D. Mateos, R.C. Myers and D.J. Winters, Meson spectroscopy in AdS/CFT with flavour, JHEP 07 (2003) 049 [hep-th/0304032] [SPIRES].

[9] J. Erdmenger, N. Evans, I. Kirsch and E. Threlfall, Mesons in Gauge/Gravity Duals - A Review, Eur. Phys. J. A 35 (2008) 81 [arXiv:0711.4467] [SPIRES].

[10] J. Babington, J. Erdmenger, N.J. Evans, Z. Guralnik and I. Kirsch, Chiral symmetry breaking and pions in non-supersymmetric gauge / gravity duals, Phys. Rev. D 69 (2004) 066007 [hep-th/0306018] [SPIRES].

[11] I. Kirsch, Generalizations of the AdS/CFT correspondence, Fortsch. Phys. 52 (2004) 727 [hep-th/0406274] [SPIRES].

[12] D. Mateos, R.C. Myers and R.M. Thomson, Thermodynamics of the brane, JHEP 05 (2007) 067 [hep-th/0701132] [SPIRES].

[13] C. Hoyos-Badajoz, K. Landsteiner and S. Montero, Holographic Meson Melting, JHEP 04 (2007) 031 [hep-th/0612169] [SPIRES].

[14] R.C. Myers, A.O. Starinets and R.M. Thomson, Holographic spectral functions and diffusion constants for fundamental matter, JHEP 11 (2007) 091 [arXiv:0706.0162] [SPIRES].

[15] D. Mateos and L. Patino, Bright branes for strongly coupled plasmas, JHEP 11 (2007) 025 [arXiv:0709.2168] [SPIRES].

[16] S. Kobayashi, D. Mateos, S. Matsuura, R.C. Myers and R.M. Thomson, Holographic phase transitions at finite baryon density, JHEP 02 (2007) 016 [hep-th/0611099] [SPIRES].

[17] D. Mateos, S. Matsuura, R.C. Myers and R.M. Thomson, Holographic phase transitions at finite chemical potential, JHEP 11 (2007) 085 [arXiv:0709.1225] [SPIRES].

[18] S. Nakamura, Y. Seo, S.-J. Sin and K.P. Yogendran, A new phase at finite quark density from AdS/CFT, J. Korean Phys. Soc. 52 (2008) 1734 [hep-th/0611021] [SPIRES].

[19] S. Nakamura, Y. Seo, S.-J. Sin and K.P. Yogendran, Baryon-charge Chemical Potential in AdS/CFT, Prog. Theor. Phys. 120 (2008) 51 [arXiv:0708.2818] [SPIRES].

[20] A. Karch and A. O'Bannon, Holographic Thermodynamics at Finite Baryon Density: Some Exact Results, JHEP 11 (2007) 074 [arXiv:0709.0570] [SPIRES].

[21] T. Faulkner and H. Liu, Condensed matter physics of a strongly coupled gauge theory with quarks: some novel features of the phase diagram, arXiv:0812.4278 [SPIRES].

[22] J. Erdmenger, M. Kaminski, P. Kerner and F. Rust, Finite baryon and isospin chemical potential in AdS/CFT with flavor, JHEP 11 (2008) 031 [arXiv:0807.2663] [SPIRES].

[23] R.C. Myers and A. Sinha, The fast life of holographic mesons, JHEP 06 (2008) 052 [arXiv: 0804.2168] [SPIRES].

[24] J. Mas, J.P. Shock, J. Tarrio and D. Zoakos, Holographic Spectral Functions at Finite Baryon Density, JHEP 09 (2008) 009 [arXiv:0805.2601] [SPIRES].

[25] A. Paredes, K. Peeters and M. Zamaklar, Mesons versus quasi-normal modes: undercooling and overheating, JHEP 05 (2008) 027 [arXiv: 0803.0759] [SPIRES].

[26] K. Binder, Theory of first-order phase transitions, Rep. Prog. Phys 50 (1987) 783. 
[27] I. Amado, C. Hoyos-Badajoz, K. Landsteiner and S. Montero, Hydrodynamics and beyond in the strongly coupled $N=4$ plasma, JHEP 07 (2008) 133 [arXiv:0805.2570] [SPIRES].

[28] I. Amado, C. Hoyos-Badajoz, K. Landsteiner and S. Montero, Residues of Correlators in the Strongly Coupled $N=4$ Plasma, Phys. Rev. D 77 (2008) 065004 [arXiv:0710.4458] [SPIRES].

[29] F. Denef, S.A. Hartnoll and S. Sachdev, Black hole determinants and quasinormal modes, arXiv:0908.2657 [SPIRES].

[30] J. Erdmenger, M. Kaminski and F. Rust, Holographic vector mesons from spectral functions at finite baryon or isospin density, Phys. Rev. D 77 (2008) 046005 [arXiv:0710.0334] [SPIRES].

[31] C.P. Herzog, P. Kovtun, S. Sachdev and D.T. Son, Quantum critical transport, duality and M-theory, Phys. Rev. D 75 (2007) 085020 [hep-th/0701036] [SPIRES].

[32] I. Amado, C. Hoyos-Badajoz, K. Landsteiner and S. Montero, Absorption Lengths in the Holographic Plasma, JHEP 09 (2007) 057 [arXiv: 0706. 2750] [SPIRES].

[33] A.S. Miranda, J. Morgan and V.T. Zanchin, Quasinormal modes of plane-symmetric black holes according to the AdS/CFT correspondence, JHEP 11 (2008) 030 [arXiv:0809.0297] [SPIRES].

[34] R.C. Myers and M.C. Wapler, Transport Properties of Holographic Defects, JHEP 12 (2008) 115 [arXiv: 0811.0480] [SPIRES].

[35] M. Kaminski, K. Landsteiner, J. Mas, J.P. Shock and J. Tarrio, Holographic Operator Mixing and Quasinormal Modes on the Brane, arXiv:0911.3610 [SPIRES].

[36] J. Morgan, V. Cardoso, A.S. Miranda, C. Molina and V.T. Zanchin, Gravitational quasinormal modes of AdS black branes in d spacetime dimensions, JHEP 09 (2009) 117 [arXiv: 0907.5011] [SPIRES].

[37] P. G. Debenedetti and F. H. Stillinger, Supercooled liquids and the glass transition, Nature 410 (2001) 259.

[38] M. Kaminski, Holographic quark gluon plasma with flavor, Fortsch. Phys. 57 (2009) 3 [arXiv: 0808.1114] [SPIRES].

[39] N. Iqbal and H. Liu, Universality of the hydrodynamic limit in AdS/CFT and the membrane paradigm, Phys. Rev. D 79 (2009) 025023 [arXiv:0809.3808] [SPIRES].

[40] G.T. Horowitz and M.M. Roberts, Zero Temperature Limit of Holographic Superconductors, JHEP 11 (2009) 015 [arXiv: 0908.3677] [SPIRES].

[41] M. Ammon, J. Erdmenger, M. Kaminski and P. Kerner, Flavor Superconductivity from Gauge/Gravity Duality, JHEP 10 (2009) 067 [arXiv:0903.1864] [SPIRES].

[42] M. Ammon, J. Erdmenger, M. Kaminski and P. Kerner, Superconductivity from gauge/gravity duality with flavor, Phys. Lett. B 680 (2009) 516 [arXiv:0810.2316] [SPIRES].

[43] A. Núñez and A.O. Starinets, AdS/CFT correspondence, quasinormal modes and thermal correlators in $N=4$ SYM, Phys. Rev. D 67 (2003) 124013 [hep-th/0302026] [SPIRES].

[44] W.H. Press, S.A. Teukolsky, W.T. Vetterling and B.P. Flannery, Numerical Recipes: The Art of Scientific Computing, Cambridge University Press, New York U.S.A. (2007) http://www.nr.com.

[45] J. W. Eaton, GNU Octave Manual, Network Theory Limited, Bristol U.K. (2002) http://www.gnu.org/software/octave/. 